\title{
Counting statistics for noninteracting fermions in a $d$-dimensional potential
}

\author{
Naftali R. Smith, ${ }^{1}$ Pierre Le Doussal, ${ }^{2}$ Satya N. Majumdar, ${ }^{1}$ and Grégory Schehr ${ }^{1}$ \\ ${ }^{1}$ LPTMS, CNRS, Univ. Paris-Sud, Université Paris-Saclay, 91405 Orsay, France \\ ${ }^{2}$ CNRS-Laboratoire de Physique Théorique de l'Ecole Normale Supérieure, 24 rue Lhomond, 75231 Paris Cedex, France
}

(Dated: March 19, 2021)

\begin{abstract}
We develop a first-principle approach to compute the counting statistics in the ground-state of $N$ noninteracting spinless fermions in a general potential in arbitrary dimensions $d$ (central for $d>1$ ). In a confining potential, the Fermi gas is supported over a bounded domain. In $d=1$, for specific potentials, this system is related to standard random matrix ensembles. We study the quantum fluctuations of the number of fermions $\mathcal{N}_{\mathcal{D}}$ in a domain $\mathcal{D}$ of macroscopic size in the bulk of the support. We show that the variance of $\mathcal{N}_{\mathcal{D}}$ grows as $N^{(d-1) / d}\left(A_{d} \log N+B_{d}\right)$ for large $N$, and obtain the explicit dependence of $A_{d}, B_{d}$ on the potential and on the size of $\mathcal{D}$ (for a spherical domain in $d>1$ ). This generalizes the free-fermion results for microscopic domains, given in $d=1$ by the Dyson-Mehta asymptotics from random matrix theory. This leads us to conjecture similar asymptotics for the entanglement entropy of the subsystem $\mathcal{D}$, in any dimension, supported by exact results for $d=1$.
\end{abstract}

An important concept to study quantum noise and correlations in many body fermionic systems is the counting statistics (CS), which characterises the fluctuations of the number of particles $\mathcal{N}_{\mathcal{D}}$ inside a domain $\mathcal{D}$. Applications include shot noise [1], quantum transport [2,3], quantum dots $[4,5]$, spin and fermionic chains [6-9], trapped fermions $[10,11]$. In the related context of random matrix theory (RMT), the statistics of the number of eigenvalues in an interval also generated a lot of interest [6,1223]. The CS is particularly important for noninteracting fermions because of its connection [24-27] to the bipartite entanglement entropy (EE) of the subsystem $\mathcal{D}$ with its complement $\overline{\mathcal{D}}$. The EE is a highly non local quantity, much studied in the context of quantum information [28, 29], conformal field theory [30-32], topological phases [33], quantum phase transitions [34, 35], or quantum spin chains $[36,37]$. Both the CS and the EE are difficult to compute analytically, in particular in the presence of an external potential. There exist however standard results for free fermions, in the absence of external potential. In this case, at zero temperature, both the variance of $\mathcal{N}_{\mathcal{D}}$ and the EE grow as $\sim R^{d-1} \log R$ with the typical size $R$ of the domain $\mathcal{D}$ [38-45].

In cold Fermi gases [46], the quantum microscopes [4749] allow to take an instantaneous "picture" and measure the counting statistics. In experiments the fermions are in a trapping potential, of tunable shape and interaction $[46,50]$. It is thus important to calculate both the CS and the $\mathrm{EE}$ in an inhomogeneous background, for which very few analytical results exist even for noninteracting fermions, apart from the $d=1$ harmonic oscillator [32, $51,52]$, and the rotating harmonic trap in $d=2$ [53].

There has been recent progress to describe noninteracting spinless fermions in traps in $d$ dimensions [11]. In $d=1$, for a single particle Hamiltonian $\hat{H}=\frac{p^{2}}{2}+V(x)$ (in units $\hbar=m=1$ ), there is a useful connection with random matrices for a few specific potentials $V(x)$. The many body ground state wavefunction $\Psi_{0}$ of $N$ fermions is a Slater determinant with all energy levels of $\hat{H}$ occupied up to the Fermi energy $\mu$, a function of $N$. The quantum joint probability $\left|\Psi_{0}\right|^{2}$ of the positions $\left\{x_{j}\right\}$ of the $N$ fermions, maps onto the joint probability for the eigenvalues $\left\{\lambda_{j}\right\}$ of random matrices of size $N \times N$. For the harmonic oscillator (HO), $V(x)=\frac{x^{2}}{2}$, the random matrix is Hermitian from the Gaussian unitary ensemble (GUE). At large $N$, the mean fermion density, i.e., the quantum average $\rho(x)=\left\langle\sum_{i} \delta\left(x-x_{i}\right)\right\rangle$, has support $\left[x^{-}, x^{+}\right]$, with $x^{ \pm} \simeq \pm \sqrt{2 N}$. In the bulk, i.e., away from the edges $x^{ \pm}$, it takes the semi-circle form $\rho(x) \simeq \rho^{\text {bulk }}(x)=k_{F}(x) / \pi$, where $k_{F}(x)=\sqrt{2 \mu-x^{2}}$ is the local Fermi momentum, and in this case $\mu \simeq N$. There are two natural length scales, the microscopic one of order the inter-particle distance $\sim 1 / k_{F}(x)$, and the macroscopic one of order $x^{+}-x^{-}$. For an interval $\mathcal{D}=[a, b]$ of microscopic size, it is well known from standard results of RMT [54, 55] that for $\sqrt{N}|b-a|=O(1) \gg 1$ the variance behaves as $[6,12-14,17,20-22]$

$$
\operatorname{Var} \mathcal{N}_{[a, b]} \simeq \frac{1}{\pi^{2}}\left[\log \left(\sqrt{2 N-a^{2}}|b-a|\right)+c_{2}\right]
$$

with $c_{2}=\gamma_{E}+1+\log 2$, where $\gamma_{E}$ is Euler's constant. The fermion/eigenvalue correlations can be expressed as determinants of a central object called the kernel, which depends on $V(x)$, see below. At microscopic scales, the kernel takes a universal scaling form, called the sine-kernel, independent of the (smooth) potential, which leads to (1). However, except for free fermions on the infinite line, it does not apply when both $a, b$ are well separated in the bulk. For the HO, some results in that regime were obtained in $[20,21]$ using a Coulomb gas method, and for the GUE in the math literature [56-59].

Despite recent advances a general framework is still lacking for computing the counting statistics and entanglement entropy for noninteracting fermions in general potential and arbitrary dimension. In this Letter we provide a first principle approach to compute these quantities in $d=1$ for a general potential $V(x)$, and in $d>1$ for a general central potential. Our method recovers the existing results in various special cases, see below. 
Let us summarize our main results. For a confining potential in $d=1$, such that the bulk density $k_{F}(x) / \pi$, $k_{F}(x)=\sqrt{2(\mu-V(x))}$, has a single support $\left[x^{-}, x^{+}\right]$, we obtain an explicit formula for $\operatorname{Var} \mathcal{N}_{[a, b]}$, with $a, b$ well separated in the bulk, $|a-b| \gg 1 / k_{F}(a)$. In the limit $N \gg 1$ (i.e., $\mu \gg 1$ ) where $N \simeq \int_{x^{-}}^{x^{+}} \frac{d x}{\pi} k_{F}(x)$

$$
\begin{aligned}
& \left(2 \pi^{2}\right) \operatorname{Var} \mathcal{N}_{[a, b]}=2 \log \left(2 k_{F}(a) k_{F}(b) \int_{x^{-}}^{x^{+}} \frac{d z}{\pi k_{F}(z)}\right) \\
& +\log \left(\frac{\sin ^{2} \frac{\theta_{a}-\theta_{b}}{2}}{\sin ^{2} \frac{\theta_{a}+\theta_{b}}{2}}\left|\sin \theta_{a} \sin \theta_{b}\right|\right)+2 c_{2}+o(1) \\
& \text { where } \quad \theta_{x}=\pi \frac{\int_{x^{-}}^{x} d z / k_{F}(z)}{\int_{x^{-}}^{x^{+}} d z / k_{F}(z)},\left\{\begin{array}{l}
\theta_{x^{-}}=0 \\
\theta_{x^{+}}=\pi
\end{array}\right.
\end{aligned}
$$

We then consider noninteracting fermions in a general central potential in $d$ dimension, with single particle Hamiltonian $\hat{H}=\frac{\mathbf{p}^{2}}{2}+V(r)$, where $r=|\mathbf{x}|$. We obtain the variance $\operatorname{Var} \mathcal{N}_{\mathcal{D}}$ for any rotationally invariant domain $\mathcal{D}$. For instance, for the HO, $V(r)=\frac{1}{2} r^{2}$, the support of the density is the ball of radius $\sqrt{2 \mu}$, and for a sphere of macroscopic radius $R=\tilde{R} \sqrt{2 \mu}$ we obtain for large $\mu$, with fixed $\tilde{R} \in[0,1[$

$$
\begin{aligned}
\operatorname{Var} \mathcal{N}_{\mathcal{D}} & =\mu^{d-1}\left[A_{d}(\tilde{R}) \log \mu+B_{d}(\tilde{R})+o(1)\right] \\
A_{d}(\tilde{R}) & =\frac{1}{\pi^{2} \Gamma(d)}\left(2 \tilde{R} \sqrt{1-\tilde{R}^{2}}\right)^{d-1}
\end{aligned}
$$

and $B_{d}(\tilde{R})$ is given below for $d=2$ in $(26)$ and for $d=$ 3 in (27). As seen from the comparison to simulations in Fig. 1 (see [60] for details on the simulations), the prediction in (4) for a disk in $d=2$ is already excellent for $\mu=100$ (it is crucial to include the sub-leading term $\left.B_{d}(\tilde{R})\right)$. In the microscopic limit $\tilde{R} \rightarrow 0$ we obtain

$$
\operatorname{Var} \mathcal{N}_{\mathcal{D}} \simeq \frac{1}{\pi^{2} \Gamma(d)}\left(k_{F} R\right)^{d-1}\left[\log \left(k_{F} R\right)+b_{d}\right],
$$

where $k_{F}=\sqrt{2 \mu}$. The leading term reproduces the free fermion result [38-43] for a sphere and we further obtain the subleading term

$$
b_{d}=2 \log 2-\frac{\gamma_{E}}{2}+1-\frac{3}{2} \psi^{(0)}\left(\frac{d+1}{2}\right),
$$

$\psi^{(0)}(x)$ being the di-gamma function. These results lead us to the conjecture (34) for the entanglement entropy of the subsystem $\mathcal{D}$ in any dimension for arbitrary smooth central potential, corroborated by exact results in $d=1$.

Let us start with fermions on the infinite line in $d=$ 1. It is useful to introduce the height field $h(x)$ [61], also called the "index" in RMT [15, 16, 18, 19], and its two-point covariance function $H(x, y)$, from which the variance of $\mathcal{N}_{\mathcal{D}}$ for any interval $\mathcal{D}=[a, b]$ is obtained as

$$
\begin{aligned}
& h(x)=\mathcal{N}_{]-\infty, x]} \quad, \quad H(x, y)=\operatorname{Cov}[h(x), h(y)], \\
& \operatorname{Var} \mathcal{N}_{[a, b]}=H(a, a)+H(b, b)-2 H(a, b)
\end{aligned}
$$

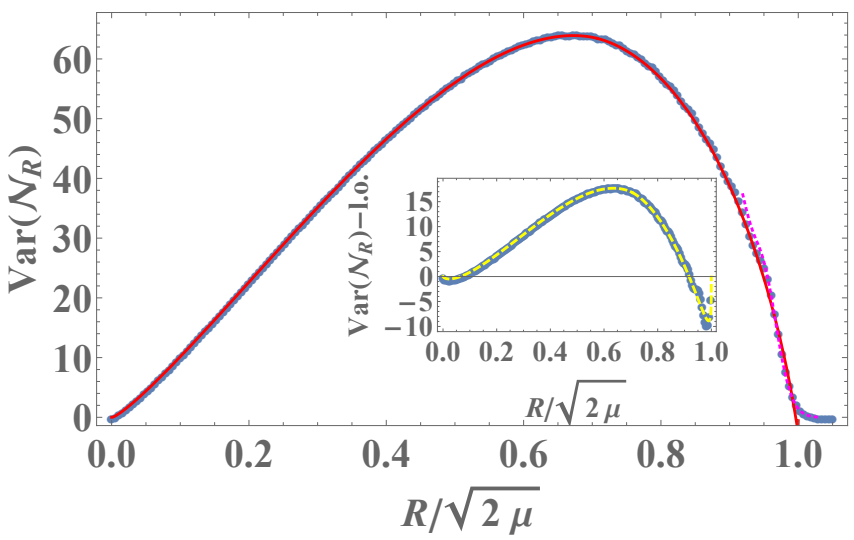

FIG. 1. Variance of $\mathcal{N}_{\mathcal{D}}=\mathcal{N}_{R}$ for a disk of radius $R$ in $d=2$, plotted vs $\tilde{R}=R / \sqrt{2 \mu}$ for $\mu=100$ corresponding to $N=\mu(\mu+1) / 2=5050$. The simulations (symbols) [60] show excellent agreement with our predictions: In the bulk, with (4) (solid line), where $A_{2}(\tilde{R})$ is given in $(5)$ and $B_{2}(\tilde{R})$ in (26), and near the edge $\tilde{R}=1$, with the scaling form (28) (dotted line). Inset: the sub-leading term $B_{2}(\tilde{R})$ plotted vs $\tilde{R}$ (dashed line), compared to the simulations (symbols), the leading term $A_{2}(\tilde{R}) \mu \log \mu$ being subtracted from the variance.

with $\operatorname{Var} \mathcal{N}_{-\infty, a]}=\operatorname{Var} \mathcal{N}_{[a,+\infty[}=H(a, a)$, for a semi infinite interval [62].

For $N$ noninteracting fermions the correlation functions are obtained from the kernel

$$
K_{\mu}(x, y)=\sum_{k=1}^{N} \psi_{k}^{*}(x) \psi_{k}(y)
$$

where the $\psi_{k}(x)$ are the eigenstates of $\hat{H}=\frac{p^{2}}{2}+V(x)$. We will denote $\left\{\epsilon_{k}\right\}_{k=1,2, \ldots}$ the eigenenergies in increasing order. The mean density is $\rho(x)=K_{\mu}(x, x)$, and the $n$ point correlation is given by $\operatorname{det}_{n \times n} K_{\mu}\left(x_{i}, x_{j}\right)$ (see e.g. [11]). This leads to the exact relation [60]

$$
K_{\mu}(x, y)^{2}=-\partial_{x} \partial_{y} H(x, y)+\delta(x-y) \rho(x)
$$

from which we determine the height field covariance (8).

We now obtain an estimate of $K_{\mu}(x, y)^{2}$, and of $H(x, y)$, valid anywhere in the bulk in the large $N$ limit. In this regime, the sum over $k$ in (10) is dominated by $k \gg 1$ [63]. One can thus use the WKB asymptotics $[64,65]$

$$
\psi_{k}(x) \simeq \frac{C_{k}}{\left[2\left(\epsilon_{k}-V(x)\right)\right]^{1 / 4}} \sin \left(\phi_{k}(x)+\frac{\pi}{4}\right)
$$

where $\phi_{k}(x)=\int_{x^{-}}^{x} d z \sqrt{2\left(\epsilon_{k}-V(z)\right)}$ and $C_{k}^{2}=\frac{2}{\pi} \frac{d \epsilon_{k}}{d k}$ is a normalization [66, 67]. Inserting (12) in (10), we relabel $k=N-m$ around the Fermi energy $\mu=\epsilon_{N}$. Noting that the phase $\phi_{N}(x)$ at large $N$ is also very large, we can expand $\phi_{N-m}(x)=\phi_{N}(x)-m \frac{d \phi_{N}(x)}{d N}+o(1)=$ $\phi_{N}(x)-m \theta_{x}+o(1)$, where $\theta_{x}$ is given in (3), using $\frac{d N}{d \mu} \simeq \int_{x^{-}}^{x^{+}} \frac{d x}{\pi k_{F}(x)}$. Performing the geometric sum over 
$m$ we obtain

$$
K_{\mu}(x, y) \simeq \frac{d \mu / d N}{2 \pi \sqrt{k_{F}(x) k_{F}(y)}} \sum_{\sigma= \pm 1} \frac{\sin \left(\tilde{\phi}_{N}(x)-\sigma \tilde{\phi}_{N}(y)\right)}{\sin \left(\left(\theta_{x}-\sigma \theta_{y}\right) / 2\right)}
$$

with $\tilde{\phi}_{N}(x)=\phi_{N}(x)+O(1)$. In Eq. (13) the sine terms oscillate on microscopic scales. For $|x-y| \sim 1 / k_{F}(x)$ the term $\sigma=1$ dominates [68]. Using $\tilde{\phi}_{N}^{\prime}(x) \simeq k_{F}(x)$ and $\frac{d \theta_{x}}{d x}=\frac{d \mu}{d N} \frac{1}{k_{F}(x)}$, one recovers the sine-kernel

$$
K_{\mu}(x, y) \simeq \frac{\sin \left(k_{F}(x)|x-y|\right)}{\pi|x-y|}
$$

valid on microscopic scales. On the other hand, for $x, y$ well separated on macroscopic scales in the bulk $] x^{-}, x^{+}[$, taking the square of (13), one can neglect the cross term and replace the $\sin ^{2}$ by $1 / 2$, leading to

$$
K_{\mu}(x, y)^{2} \simeq \frac{(d \mu / d N)^{2}}{2 \pi^{2} k_{F}(x) k_{F}(y)} \frac{1-\cos \left(\theta_{x}\right) \cos \left(\theta_{y}\right)}{\left(\cos \theta_{x}-\cos \theta_{y}\right)^{2}}
$$

up to fast oscillating terms averaging to zero on scales larger than microscopic. Note that Eq. (15) is valid for any smooth potential: for the $\mathrm{HO}$ we also derived these estimates using the Plancherel-Rotach asymptotics for the Hermite polynomials [60]. Having obtained $K_{\mu}(x, y)^{2}$ in the two regimes, we use (11) to compute the height correlator.

(i) For $x, y$ well separated in the bulk, i.e., $|x-y| \gg$ $1 / k_{F}(x)$, the 2 -point height covariance is given by

$H(x, y) \simeq \frac{1}{2 \pi^{2}}\left(\log \left|\sin \frac{\theta_{x}+\theta_{y}}{2}\right|-\log \left|\sin \frac{\theta_{x}-\theta_{y}}{2}\right|\right)$

up to $o(1)$ terms at large $\mu$. One checks that (16) is consistent with (15) and (11) (in this regime the $\delta$ function does not contribute). Using (3), the right hand side (r.h.s.) in (16) vanishes when $x$ is in the bulk and $y$ reaches an edge $y=x^{ \pm}$, and for $\left.y \notin\right] x^{-}, x^{+}[$, $H(x, y) \simeq o(1)[60]$. The r.h.s. in (16) coincides with the correlator of the 2D Gaussian free field (GFF) in the upper-half plane (with Dirichlet boundary conditions) along part of a circle $z=e^{i \theta_{x}}$, thus extending the result of [57] for the GUE/HO [69]. Similar connections to the GFF also emerge in recent approaches using inhomogeneous bosonization [32, 72-74].

(ii) On microscopic scales, $|x-y| \sim 1 / k_{F}(x)$, one uses the sine kernel (14) in the left hand side of (11). The integration constants are fixed so that $H(x, y)$ for $|x-y| \gg 1 / k_{F}(x)$ matches with the limit $y \rightarrow x$ in (16) leading to

$H(x, y) \simeq \frac{1}{2 \pi^{2}}\left[U\left(k_{F}(x)|x-y|\right)+\log \frac{2 k_{F}(x) \sin \theta_{x}}{d \theta_{x} / d x}\right]$

where

$$
U(z)=\operatorname{Ci}(2 z)+2 z \operatorname{Si}(2 z)-\log z+1-2 \sin ^{2}(z)-\pi z,
$$

with $U(z \gg 1)=-\log z+o(1)$ and $U(z \ll 1)=1+$ $\gamma_{E}+\log 2-\pi z+z^{2}+o\left(z^{2}\right)$. One checks, using $U^{\prime \prime}(z)=$ $2 \sin ^{2} z / z^{2}$, that (17) is consistent with (11) (including the delta function) and $K_{\mu}$ given by the sine kernel (14), since $k_{F}(x) \simeq k_{F}(y)$ on microscopic scales. Using (9), it leads to the Dyson Mehta behavior

$$
\begin{aligned}
\pi^{2} \operatorname{Var} \mathcal{N}_{[a, b]} & \simeq U(0)-U\left(k_{F}(a)|a-b|\right) \\
& \simeq \log k_{F}(a)|a-b|+c_{2} .
\end{aligned}
$$

From (16), (17) and (9), we obtain our result (2) as well as, for any $a$ in the bulk

$H(a, a)=\operatorname{Var} \mathcal{N}_{[a,+\infty[} \simeq \frac{1}{2 \pi^{2}}\left(\log \frac{2 k_{F}(a)^{2} \sin \theta_{a}}{d \mu / d N}+c_{2}\right)$.

Expanding (20) for $a \rightarrow x^{+}, a<x^{+}$, one obtains [60] $H(a, a) \simeq \frac{1}{2 \pi^{2}}\left(\frac{3}{2} \log (-\hat{a})+c_{2}+2 \log 2\right)$ for $-\hat{a} \gg 1$. Here the edge scaling variable is $\hat{a}=\left(a-x^{+}\right) / w_{N}$, and $w_{N}=\left(2 V^{\prime}\left(x^{+}\right)\right)^{-1 / 3}$, the width of the edge regime [11], appears naturally. Inside the edge regime, i.e., for $\hat{a}=O(1), H(a, a) \simeq \frac{1}{2} \mathcal{V}_{2}(\hat{a})$, where the scaling function $\mathcal{V}_{2}$ was defined in $[20,21]$ for the HO, but is universal for a smooth potential [60]. The matching with the bulk for $\hat{a} \rightarrow-\infty$ obtained above agrees with known results for the HO/GUE [20, 75, 77].

For the $\mathrm{HO} x^{ \pm}= \pm \sqrt{2 \mu}, \theta_{x}=\arccos (-x / \sqrt{2 \mu})$ and (16) agrees with the rigorous results for the GUE [57]. In this case (2) gives a general result [60] which agrees with known results in special cases [15, 16, 20, 21, 58].

Another important example is the inverse square well $V(x)=\frac{x^{2}}{2}+\frac{\alpha(\alpha-1)}{2 x^{2}}$ for $x>0$ and $\alpha \geq 1 / 2$. It corresponds [60] to the Wishart-Laguerre unitary ensemble (LUE) of random matrices [78] with the correspondence between fermion positions $x_{j}$ and eigenvalues $\lambda_{j} \sim x_{j}^{2}$ [79-81]. One has $\mu=2 N+\alpha+1 / 2$, hence $d \mu / d N \simeq 2$ and $\cos \theta_{x}=$ $\frac{\mu-x^{2}}{\sqrt{\mu^{2}-\alpha(\alpha-1)}}$. We focus on the interval $[0, a]$ and scale both $a=O(\sqrt{\mu})$ and $\alpha=O(\mu)$ in the large $\mu$ limit. This scaling, used below for $d$-dimensional central potentials, is also the standard large- $N$ limit for Wishart matrices. Setting $\tilde{a}=a / \sqrt{2 \mu}$ and $\lambda=\alpha / \mu$, one obtains from (20) in the bulk $\left|2 \tilde{a}^{2}-1\right|<\sqrt{1-\lambda^{2}}$

$2 \pi^{2} \operatorname{Var} \mathcal{N}_{[0, a]}^{\mathrm{LUE}} \simeq \log (\mu)+\log \left(4 \tilde{a} \frac{\left(1-\tilde{a}^{2}-\frac{\lambda^{2}}{4 \tilde{a}^{2}}\right)^{3 / 2}}{\left(1-\lambda^{2}\right)^{1 / 2}}\right)+c_{2}$

with the superscript LUE added for later convenience. A similar result was recently reported in the mathematics literature $[82,83]$. The result (16) also agrees with rigorous GFF results for the LUE [85, 86]. We have extended these results to other cases related to RMT [60].

We now address a central potential $V(r)$ in $d>1$ and focus on the number of fermions $\mathcal{N}_{R}$ in a spherical domain $\mathcal{D}$ of radius $R$ centered at the origin. The single particle Hamiltonian $\hat{H}$ commutes with the angular momentum 
$\hat{\boldsymbol{L}}$, and with $\hat{\boldsymbol{L}}^{2}$ of eigenvalues $\ell(\ell+d-2), \ell=0,1, \ldots$, defining the sector of angular momentum $\ell$. The eigenstates of $\hat{H}$ are obtained from those of a collection of 1D radial problems $\hat{H}_{\ell}=-\frac{1}{2} \partial_{r}^{2}+V_{\ell}(r), r \geq 0$, with potentials $[80,87]$

$$
V_{\ell}(r)=V(r)+\frac{\left(\ell+\frac{d-3}{2}\right)\left(\ell+\frac{d-1}{2}\right)}{2 r^{2}}
$$

and eigenenergies $\epsilon_{n, \ell}$, each with degeneracy $g_{d}(\ell)$, which behaves as $g_{d}(\ell \gg 1) \simeq \frac{2 \ell^{d-2}}{\Gamma(d-1)}$. We consider the $N$ fermion ground state where all levels with $\epsilon_{n, \ell} \leq \mu$ are filled. In each sector $\ell$, the levels $n=1, \ldots, m_{\ell}$ are occupied, with $N=\sum_{\ell} g_{d}(\ell) m_{\ell}$, with $m_{\ell}=0$ for $\ell>\ell_{\max }(\mu)$. Remarkably, we show [60] that the quantum joint probability of the radial positions $\left\{r_{i}\right\}_{i=1, \ldots, N}$ of the fermions decouples into a symmetrized product over the angular sectors. As a consequence, the cumulants $\left\langle\mathcal{N}_{R}^{p}\right\rangle^{c}$ for $p \geq 1$ are simply sums over the angular sectors as

$$
\left\langle\mathcal{N}_{R}^{p}\right\rangle^{c}=\sum_{\ell=0}^{\ell_{\max }(\mu)} g_{d}(\ell)\left\langle\mathcal{N}_{[0, R]}^{p}\right\rangle_{\ell}^{c}
$$

where $\left\langle\mathcal{N}_{[0, R]}^{p}\right\rangle_{\ell}^{c}$ are the cumulants of $\mathcal{N}_{[0, R]}$ for the $1 \mathrm{~d}$ potential $V_{\ell}(r)$ in (22) with $m_{\ell}$ fermions. In the large $\mu$ limit, the sum in (23) is dominated by large values of $\ell$ and $m_{\ell}$, and, for $p>1$, is effectively cut-off at $\ell_{c}(\mu, R) \simeq$ $R k_{F}(R) \leq \ell_{\max }$, where $k_{F}(r)=\sqrt{2(\mu-V(r))}$. This allows us to use our results in $1 \mathrm{~d}$ and to obtain the variance of $\mathcal{N}_{R}$ for a general central potential, see [60].

We discuss here the HO $V(r)=r^{2} / 2$, for which the density has a spherical support, with $\rho^{\text {bulk }}(r) \sim$ $\left(2 \mu-r^{2}\right)^{d / 2}$, and an edge at $r=r_{e}=\sqrt{2 \mu}$ [88]. In this case $V_{\ell}(r)$ in (22) is the inverse square well studied above with $\alpha=\ell+\frac{d-1}{2}$. For large $\mu$, the occupation numbers $m_{\ell}$ are determined by $\epsilon_{m_{\ell}, \ell} \simeq 2 m_{\ell}+\ell \simeq \mu$. Hence, defining $\lambda=\ell / \mu$, one has $m_{\ell} \simeq \frac{\mu}{2}(1-\lambda)$ for $\lambda<1$ and $m_{\ell}=0$ for $\lambda>1$. The total number of fermions is thus

$$
N \simeq \frac{\mu^{d}}{\Gamma(d-1)} \int_{0}^{1} d \lambda(1-\lambda) \lambda^{d-2}=\frac{\mu^{d}}{\Gamma(d+1)} .
$$

Substituting the result (21) with $a=R$, i.e., $\tilde{a}=\tilde{R}=$ $R / \sqrt{2 \mu}$, into (23) with $p=2$, and approximating the sum by an integral, one obtains, using $\ell_{c}(\mu, R) / \mu=$ $2 \tilde{R} \sqrt{1-\tilde{R}^{2}}$

$$
\operatorname{Var} \mathcal{N}_{R} \simeq \frac{2 \mu^{d-1}}{\Gamma(d-1)} \int_{0}^{2 \tilde{R} \sqrt{1-\tilde{R}^{2}}} d \lambda \lambda^{d-2} \operatorname{Var} \mathcal{N}_{[0, R]}^{\mathrm{LUE}}
$$

Performing the integral over $\lambda$ yields the result in (4) and (5) for the HO in the large $\mu$ limit. The coefficient $A_{d}(\tilde{R})$ has a maximum at $\tilde{R}=1 / \sqrt{2}$ for any $d>1$, and vanishes at the edge as $A_{d}(\tilde{R}) \sim(1-\tilde{R})^{(d-1) / 2}$. The $O\left(\mu^{d-1}\right)$ term $B_{d}$ is obtained in [60] for general $d$. For $d=2$ and $d=3$ it reads

$$
\begin{aligned}
& 2 \pi^{2} B_{2}(x)=\log \left(\frac{\left|1-2 x \sqrt{1-x^{2}}\right|}{1+2 x \sqrt{1-x^{2}}}\right) \\
& +2 x \sqrt{1-x^{2}}\left\{\log \left[\left(\frac{64 x}{1-2 x^{2}}\right)^{2}\left(1-x^{2}\right)^{3}\right]+2 \gamma_{E}-2\right\}
\end{aligned}
$$

and

$$
\begin{aligned}
& 2 \pi^{2} B_{3}(x)=\left(1-2 x^{2}\right)^{2} \log \left|1-2 x^{2}\right| \\
& \quad+4 x^{2}\left(1-x^{2}\right)\left\{\log \left[8 x\left(1-x^{2}\right)^{3 / 2}\right]+\gamma_{E}\right\} .
\end{aligned}
$$

respectively. $B_{d}(x)$ has a singularity $(1-x)^{\frac{d-1}{2}} \log (1-x)$ near the edge at $x=1$. As in $d=1$, there is an edge region of width $w_{N}=\left(2 V^{\prime}\left(r_{e}\right)\right)^{-1 / 3}$ where the variance becomes a universal function of $\hat{R}=\left(R-r_{e}\right) / w_{N}$

$$
\operatorname{Var} \mathcal{N}_{R} \simeq\left(\frac{r_{e}}{w_{N}}\right)^{d-1} \int_{0}^{\infty} \frac{d \xi \xi^{\frac{d-3}{2}}}{2 \Gamma(d-1)} \mathcal{V}_{2}(\hat{R}+\xi) .
$$

Here $\left(r_{e} / w_{N}\right)^{d-1}$ is the typical number of fermions in the edge region [11] and $\mathcal{V}_{2}$ is the above scaling function for $d=1$, defined in [20,21]. For the HO, Eq. (28) matches, for $\hat{R} \rightarrow-\infty$, the behavior of $B_{d}(x)$ for $x \rightarrow 1^{-}[60]$. Finally, the small $R$ limit corresponding to free fermions, given in the introduction, can also be obtained directly [60] using the sine-kernel analog in $d$ dimensions [11, 42].

One can ask about higher cumulants of $\mathcal{N}_{\mathcal{D}}$. In $d=$ 1 , for potentials related to RMT they can be extracted from known Fisher-Hartwig asymptotics of Hankel and Toeplitz determinants $[17,58,82,89]$. In all cases we find for $n \geq 2$ [90]

$$
\left\langle\mathcal{N}_{[a, b]}^{2 n}\right\rangle^{c}=\kappa_{2 n}+o(1), \kappa_{2 n}=(-1)^{n+1}(2 n) ! \frac{2 \zeta(2 n-1)}{n(2 \pi)^{2 n}}
$$

and $\left\langle\mathcal{N}_{[a, b]}^{2 n+1}\right\rangle^{c}=o(1)$, where $\zeta(x)$ is the Riemann zeta function. This leads to two important observations. First, from very recent results [84], Eq. (29) also holds for the potential $V_{\ell}(r) \simeq \frac{\ell^{2}}{2 r^{2}}$ even when $\ell \sim \mu$. Using our Eq. (23) we obtain [60] the cumulants of $\mathcal{N}_{R}$ for free fermions in dimension $d>1$, with $k_{F} R \gg 1$

$$
\left\langle\mathcal{N}_{R}^{2 n}\right\rangle^{c}=\frac{\left(k_{F} R\right)^{d-1}}{\Gamma(d)}\left(\kappa_{2 n}+o(1)\right), \quad n \geq 2 .
$$

Second, since (29) coincides with the results from the sine-kernel (and the Circular Unitary Ensemble) [6, 17, $22,27]$, it is natural to conjecture that these higher cumulants arise solely from fluctuations on microscopic scales and that (29) actually holds in $d=1$ for any smooth potential $V(x)$ [91]. For $d>1$, using $\ell_{c}(\mu, R) \simeq$ $R k_{F}(R)$ in Eq. (23), our conjecture leads to $\left\langle\mathcal{N}_{R}^{2 n}\right\rangle^{c}=$ $\frac{\left(k_{F}(R) R\right)^{d-1}}{\Gamma(d)}\left(\kappa_{2 n}+o(1)\right)$, a natural extension of our result for free fermions (30), where $k_{F}(R)$ now depends on 
$R$. In fact, for $V(r)=\frac{1}{2} r^{2}$ the argument is already close to being rigorous [92].

We now apply our results to the calculation of the bipartite Rényi entanglement entropy of a $d$-dimensional domain $\mathcal{D}$ with its complement $\overline{\mathcal{D}}$. It is defined for $q \geq 1$ as

$$
S_{q}(\mathcal{D})=\frac{1}{1-q} \ln \operatorname{Tr}\left[\rho_{\mathcal{D}}^{q}\right]
$$

where $\rho_{\mathcal{D}}=\operatorname{Tr}_{\overline{\mathcal{D}}}[\rho]$ is obtained by tracing out the density matrix $\rho$ of the system over $\overline{\mathcal{D}}$. For noninteracting fermions $S_{q}(\mathcal{D})$ can be expressed as a series,

$$
S_{q}(\mathcal{D})=\sum_{n \geq 1} s_{n}^{(q)}\left\langle\mathcal{N}_{\mathcal{D}}^{2 n}\right\rangle^{c}
$$

in the cumulants of $\mathcal{N}_{\mathcal{D}}$, where $s_{n}^{(q)}$ are given in [27] and $s_{1}^{(q)}=\frac{\pi^{2}}{6}\left(1+\frac{1}{q}\right)$. In $d=1$ this relation leads to the well known result for the entropy of free fermions,

$$
S_{q}^{\mathrm{ff}}([a, b]) \simeq \frac{q+1}{6 q} \log \left(2 k_{F}|a-b|\right)+E_{q}
$$

where $E_{q}$ is given in Eq. (11) in [94] (see also [36]). Our conjecture for the the higher cumulants in an arbitrary potential (central for $d>1$ ) leads to

$$
S_{q}(\mathcal{D})=\frac{\pi^{2}}{6} \frac{q+1}{q} \operatorname{Var} \mathcal{N}_{\mathcal{D}}+\frac{\left(k_{F}(R) R\right)^{d-1}}{\Gamma(d)}\left(\tilde{E}_{q}+o(1)\right)
$$

with $\tilde{E}_{q}=E_{q}-\frac{q+1}{6 q}\left(1+\gamma_{E}\right)$. It holds in $d>1$ for the sphere centered at the origin, and in $d=1$ for any interval $\mathcal{D}=[a, b]$ with both $a, b$ in the bulk [95]. In (34) the simple form of the second term arises from the common $R$ dependence of the cumulants of order 4 and higher. This conjecture is corroborated by the rigorous results leading to (29) in $d=1$ for the $\mathrm{HO}$, the inverse square well and the hard box [60]. It also agrees with existing results for $d=1[32,52,94,96]$. Thanks to (30), Eq. (34) is exact for free fermions in $d>1$. The leading term $S_{q}(\mathcal{D}) \propto R^{d-1} \log R$ at large $R$ is consistent with the result obtained using the Widom conjecture applied to a spherical domain [38-41, 43], and also with the rigorous proof in [97]. Here, in addition, we obtain the first correction $O\left(R^{d-1}\right)$.

In conclusion we obtained analytically the counting statistics and the entanglement entropy for $N \gg 1$ noninteracting fermions at temperature $T=0$ in a general potential in $d=1$, and a central potential in $d>1$. They depend non trivially on the shape of the potential, already at leading order in $d>1$, e.g. in (4). These results can be extended to finite $T$ [98] and it would be interesting to extend them to interacting particles, as was done recently for bosons [99].

Acknowledgments: We thank A. Borodin and C. Charlier for interesting discussions. NRS acknowledges support from the Yad Hanadiv fund (Rothschild fellowship). This research was supported by ANR grant ANR-17CE30-0027-01 RaMaTraF.
[1] L. S. Levitov and G. B. Lesovik, Charge distribution in quantum shot noise, JETP Lett. 58, 230 (1993).

[2] L. S. Levitov, H. W. Lee, G. B. Lesovik, Electron counting statistics and coherent states of electric current, J. Math. Phys. 37, 4845 (1996).

[3] I. V. Protopopov, D. B. Gutman, and A. D. Mirlin, Luttinger liquids with multiple Fermi edges: Generalized Fisher-Hartwig conjecture and numerical analysis of Toeplitz determinants, Lith. J. Phys. 52, 165 (2012).

[4] S. Gustavsson, R. Leturcq, B. Simovič, R. Schleser, T. Ihn, P. Studerus, K. Ensslin, D. C. Driscoll, A. C. Gossard, Counting statistics of single electron transport in a quantum dot, Phys. Rev. Lett. 96, 076605 (2006).

[5] C. W. Groth, B. Michaelis, C. W. J. Beenakker, Counting statistics of coherent population trapping in quantum dots, Phys. Rev. B 74, 125315 (2006).

[6] A. G. Abanov, D. A. Ivanov, Y. Qian, Quantum fluctuations of one-dimensional free fermions and FisherHartwig formula for Toeplitz determinants, J. Phys. A: Math. Theor. 44, 485001 (2011).

[7] D. A. Ivanov and A. G. Abanov, Characterizing correlations with full counting statistics: Classical Ising and quantum XY spin chains, Phys. Rev. E 87, 022114 (2013).

[8] V. Eisler and Z. Rácz, Full Counting Statistics in a Propagating Quantum Front and Random Matrix Spectra, Phys. Rev. Lett. 110, 060602 (2013).

[9] O. Gamayun, O. Lychkovskiy and J.S. Caux, Fredholm determinants, full counting statistics and Loschmidt echo for domain wall profiles in one-dimensional free fermionic chains, SciPost Physics 8, 036 (2020).

[10] V. Eisler, Universality in the full counting statistics of trapped fermions, Phys. Rev. Lett. 111, 080402 (2013).

[11] D. S. Dean, P. Le Doussal, S. N. Majumdar and G. Schehr, noninteracting fermions at finite temperature in a d-dimensional trap: universal correlations, Phys. Rev. A 94, 063622 (2016).

[12] M. M. Fogler and B. I. Shklovskii, Probability of an eigenvalue number fluctuation in an interval of a random matrix spectrum, Phys. Rev. Lett. 74, 3312 (1995).

[13] O. Costin and J. L. Lebowitz, Gaussian fluctuation in random matrices, Phys. Rev. Lett. 75, 69 (1995).

[14] M. L. Mehta, Random matrices, Elsevier (2004).

[15] S. N. Majumdar, C. Nadal, A. Scardicchio and P. Vivo, 
Index distribution of Gaussian random matrices, Phys. Rev. Lett. 103, 220603 (2009).

[16] S. N. Majumdar, C. Nadal, A. Scardicchio and P. Vivo, How many eigenvalues of a Gaussian random matrix are positive?, Phys. Rev. E 83, 041105 (2011).

[17] P. Deift, A. R. Its, and I. Krasovsky, Asymptotics of Toeplitz, Hankel, and Toeplitz+Hankel determinants with Fisher-Hartwig singularities, Ann. Math. 174, 1243 (2011).

[18] S. N. Majumdar and P. Vivo, Number of relevant directions in principal component analysis and Wishart random matrices, Phys. Rev. Lett. 108, 200601 (2012).

[19] R. Marino, S. N. Majumdar, G. Schehr and P. Vivo, Index distribution of Cauchy random matrices, J. Phys. A: Math. Theor. 47, 055001 (2014).

[20] R. Marino, S. N. Majumdar, G. Schehr, P. Vivo, Phase transitions and edge scaling of number variance in Gaussian random matrices, Phys. Rev. Lett. 112, 254101 (2014).

[21] R. Marino, S. N. Majumdar, G. Schehr, and P. Vivo, Number statistics for $\beta$-ensembles of random matrices: Applications to trapped fermions at zero temperature, Phys. Rev. E 94, 032115 (2016).

[22] C. Charlier, Large gap asymptotics for the generating function of the sine point process, Proc. London Math. Soc. (2021), preprint arXiv:1906.11079 (2019)

[23] Y. V. Fyodorov, and P. Le Doussal, Statistics of extremes in eigenvalue-counting staircases, Phys. Rev. Lett. 124, 210602 (2020).

[24] I. Klich, Lower entropy bounds and particle number fluctuations in a Fermi sea, J. Phys. A: Math. Gen. 39, L85 (2006).

[25] I. Klich, and L. Levitov, Quantum noise as an entanglement meter, Phys. Rev. Lett. 102, 100502 (2009).

[26] H. F. Song, C. Flindt, S. Rachel, I. Klich, and K. Le Hur, Entanglement entropy from charge statistics: Exact relations for noninteracting many-body systems, Phys. Rev. B 83, 161408(R) (2011).

[27] P. Calabrese, M. Mintchev, and E. Vicari, Exact relations between particle fluctuations and entanglement in Fermi gases, Europhys. Lett. 98, 20003 (2012).

[28] A. Nahum, J. Ruhman, S. Vijay, and J. Haah, Quantum entanglement growth under random unitary dynamics, Phys. Rev. X 7, 031016 (2017).

[29] E. Cornfeld, E. Sela, and M. Goldstein, Measuring fermionic entanglement: Entropy, negativity, and spin structure, Phys. Rev. A 99, 062309 (2019).

[30] P. Calabrese, and J. Cardy, Entanglement entropy and quantum field theory, J. Stat. Mech. P06002 (2004).

[31] P. Calabrese, J. Cardy, and B. Doyon, Entanglement entropy in extended quantum systems, J. Phys. A 42, 500301 (2009).

[32] J. Dubail, J.-M. Stephan, J. Viti, and P. Calabrese, Conformal Field Theory for Inhomogeneous One-dimensional Quantum Systems: the Example of noninteracting Fermi Gases, SciPost Phys. 2, 002 (2017).

[33] L. Amico, R. Fazio, A. Osterloh, and V. Vedral, Entanglement in many-body systems, Rev. Mod. Phys. 80, 517 (2008).

[34] G. Refael, and J. E. Moore, Entanglement entropy of random quantum critical points in one dimension, Phys. Rev. Lett. 93, 260602 (2004).

[35] M. A. Metlitski, C. A. Fuertes and S. Sachdev, Entan- glement entropy in the $O(N)$ model, Phys. Rev. B 80, 115122 (2009).

[36] B. Q. Jin, and V. E. Korepin, Quantum Spin Chain, Toeplitz Determinants and Fisher-Hartwig Conjecture, J. Stat. Phys. 116, 79 (2004).

[37] J.P. Keating and F. Mezzadri, Entanglement in Quantum Spin Chains, Symmetry Classes of Random Matrices, and Conformal Field Theory, Phys.Rev.Lett. 94, 050501 (2005).

[38] H. Widom, A theorem on translation kernels in $n$ dimensions, T. Am. Math. Soc. 94 170, (1960).

[39] H. Widom, Toeplitz centennial, Operator Theory: Adv. Appl. 4, 477 (1982).

[40] H. Widom, On a class of integral operators on a halfspace with discontinuous symbol, J. Funct. Anal. 88, 166 (1990).

[41] D. Gioev, and I. Klich, Entanglement entropy of fermions in any dimension and the Widom conjecture, Phys. Rev. Lett. 96, 100503 (2006).

[42] S. Torquato, A. Scardicchio, and C. E. Zachary, Point processes in arbitrary dimension from fermionic gases, random matrix theory, and number theory, J. Stat. Mech. P11019 (2008).

[43] P. Calabrese, M. Minchev, and E. Vicari, Entanglement entropies in free-fermion gases for arbitrary dimension, EPL 97, 20009 (2012).

[44] S. Fraenkel and M. Goldstein, Symmetry resolved entanglement: exact results in $1 D$ and beyond, J. Stat. Mech. (2020) 033106

[45] M. T. Tan and S. Ryu, Particle number fluctuations, Rényi entropy, and symmetry-resolved entanglement entropy in a two-dimensional Fermi gas from multidimensional bosonization, Phys. Rev. B 101, 235169 (2020).

[46] I. Bloch, J. Dalibard and W. Zwerger, Many-body physics with ultracold gases, Rev. Mod. Phys. 80, 885 (2008).

[47] L. W. Cheuk, M. A. Nichols, M. Okan, T. Gersdorf, R.Vinay, W. Bakr, T. Lompe and M. Zwierlein, Quantum-gas microscope for fermionic atoms, Phys. Rev. Lett. 114, 193001 (2015).

[48] E. Haller, J. Hudson, A. Kelly, D. A. Cotta, B. Peaudecerf, G. D. Bruce and S. Kuhr, Single-atom imaging of fermions in a quantum-gas microscope, Nat. Phys. 11, 738 (2015).

[49] M. F. Parsons, F. Huber, A. Mazurenko, C. S. Chiu, W. Setiawan, K. Wooley-Brown, S. Blatt, and M. Greiner, Site-resolved imaging of fermionic ${ }^{6} \mathrm{Li}$ in an optical lattice, Phys. Rev. Lett. 114, 213002 (2015).

[50] B. Mukherjee, Z. Yan, P. B. Patel, Z. Hadzibabic, T.Yefsah, J. Struck, M. W. Zwierlein, Homogeneous atomic Fermi gases, Phys. Rev. Lett. 118, 123401 (2017).

[51] E. Vicari, Entanglement and particle correlations of Fermi gases in harmonic traps, Phys. Rev. A 85, 062104 (2012).

[52] P. Calabrese, P. Le Doussal, and S. N. Majumdar, Random matrices and entanglement entropy of trapped Fermi gases, Phys. Rev. A 91, 012303 (2015).

[53] B. Lacroix-A-Chez-Toine, S. N. Majumdar, and G. Schehr, Rotating trapped fermions in two dimensions and the complex Ginibre ensemble: Exact results for the entanglement entropy and number variance, Phys. Rev. A 99, 021602(R) (2019).

[54] F. J. Dyson, Statistical theory of the energy levels of 
complex systems. I, J. Math. Phys. 3, 140 (1962); Statistical theory of the energy levels of complex systems. II, ibid. 3, 157 (1962); Statistical theory of the energy levels of complex systems. III, ibid. 3, 166 (1962).

[55] F. J. Dyson and M. L. Mehta, Statistical theory of the energy levels of complex systems. IV, 3, 701 (1962).

[56] Z. Bai, X. Wang, and W. Zhou, CLT for linear spectral statistics of Wigner matrices, Electron. J. Probab. 14, 2391 (2009).

[57] A. Borodin, CLT for spectra of submatrices of Wigner random matrices, Mosc. Math. J. 14, 29 (2014); A. Borodin and P. L. Ferrari, Anisotropic growth of random surfaces in $2+1$ dimensions, Commun. Math. Phys. 325, 603 (2014).

[58] C. Charlier, A. Deaño, Asymptotics for Hankel determinants associated to a Hermite weight with a varying discontinuity, SIGMA 14, 018 (2018).

[59] K. Johansson, and G. Lambert, Gaussian and nonGaussian fluctuations for mesoscopic linear statistics in determinantal processes, Ann. Probab. 46, 1201 (2018).

[60] N. R. Smith, P. Le Doussal, S. N. Majumdar, and G. Schehr, Supplemental material, which also cites [100112].

[61] F. D. M. Haldane, Effective harmonic-fluid approach to low-energy properties of one-dimensional quantum fluids, Phys. Rev. Lett. 47, 1840 (1981).

[62] For any union of disjoint intervals one has $\operatorname{Var} \mathcal{N}_{\cup_{i}\left[a_{i}, b_{i}\right]}=\sum_{i j} H\left(a_{i}, a_{j}\right)+H\left(b_{i}, b_{j}\right)-2 H\left(a_{i}, b_{j}\right)$

[63] What is meant is that the only states which are not accurately described by our WKB calculation are the few very deep low energy states, which however are not relevant in the large $N$ limit.

[64] L. D. Landau, and E. M. Lifshitz, Quantum Mechanics: Non-Relativistic Theory, 3rd ed., Vol. 3 (Pergamon, Elmsford, NY, 1977).

[65] O. Vallée and M. Soares, Airy Functions and Applications to Physics, (Imperial College Press, London, 2004).

[66] W. H. Furry, Two notes on phase-integral methods, Phys. Rev. 71, 360 (1947).

[67] The lower bound of the integral in the definition of $\phi_{k}(x)$ depends in general on $k$, but is unimportant in the argument [60].

[68] In that regime the sum over $m$ can be approximated by a continuous integral.

[69] In the edge regime it takes the form obtained in [70, 71].

[70] See section V in A. Krajenbrink, P. Le Doussal, and S. Prolhac, Systematic time expansion for the KardarParisi-Zhang equation, linear statistics of the GUE at the edge and trapped fermions, Nucl. Phys. B 936, 239 (2018).

[71] C. Charlier and T. Claeys, Large gap asymptotics for Airy kernel determinants with discontinuities, Commun. Math. Phys. 375, 1299 (2020).

[72] Y. Brun and J. Dubail, One-particle density matrix of trapped one-dimensional impenetrable bosons from conformal invariance, SciPost Phys. 2, 012 (2017); The Inhomogeneous Gaussian Free Field, with application to ground state correlations of trapped 1d Bose gases, SciPost Phys. 4, 037 (2018).

[73] J. Unterberger, Global fluctuations for $1 D$ log-gas dynamics. Covariance kernel and support, Electron. J. Probab. 24, 1 (2019)
[74] P. Ruggiero, Y. Brun, and J. Dubail, Conformal field theory on top of a breathing one-dimensional gas of hard core bosons, SciPost Phys. 6, 051 (2019).

[75] The $O(1)$ constant can further be extracted from [76]

[76] T. Bothner and B. Buckingham, Large deformations of the Tracy-Widom distribution I: non-oscillatory asymptotics, Commun. Math. Phys. 359, 223 (2018).

[77] J. Gustavsson, Gaussian fluctuations of eigenvalues in the GUE, Ann. Inst. H. Poincaré, Probab. Statist. 41, 151 (2005).

[78] P. J. Forrester, Log-Gases and Random Matrices, (London Mathematical Society monographs, Princeton University Press, Princeton, NJ, 2010)

[79] C. Nadal, and S. N. Majumdar, Nonintersecting Brownian interfaces and Wishart random matrices, Phys. Rev. E 79, 061117 (2009).

[80] D. S. Dean, P. Le Doussal, S. N. Majumdar, and G. Schehr, Statistics of the maximal distance and momentum in a trapped Fermi gas at low temperature, J. Stat. Mech. 063301 (2017).

[81] B. Lacroix-A-Chez-Toine, P. Le Doussal, S. N. Majumdar, and G. Schehr, noninteracting fermions in hardedge potentials, J. Stat. Mech. 123103 (2018).

[82] C. Charlier, Exponential Moments and Piecewise Thinning for the Bessel Point Process, Int. Math. Res. Not. (2020), preprint arXiv:1812.02188 (2018).

[83] It seems that the constant term can also be proved rigorously, see [84].

[84] C. Charlier, J. Lenells, The hard-to-soft edge transition: Exponential moments, central limit theorems and rigidity, (to be published).

[85] See definition 4.11 in A. Borodin and V. Gorin, General $\beta$-Jacobi Corners Process and the Gaussian Free Field, Commun. Pur. Appl. Math. 68, 1774 (2015).

[86] I. Dumitriu, and E. Paquette, Spectra of overlapping Wishart matrices and the Gaussian free field, Random Matrices-Theo 7, 1850003 (2018).

[87] M. Moshinsky, and Y. F. Smirnov, The Harmonic Oscillator in Modern Physics, (Contempary Concepts in Physics Vol. 9) (Amsterdam: Harwood Academic), (1996).

[88] D. S. Dean, P. Le Doussal, S. N. Majumdar, and $\mathrm{G}$. Schehr, Universal ground state properties of free fermions in a d-dimensional trap, Europhys. Lett. 112, 60001 (2015).

[89] C. Charlier, and R. Gharakhloo, Asymptotics of Hankel determinants with a Laguerre-type or Jacobi-type potential and Fisher-Hartwig singularities, preprint arXiv: 1902.08162

[90] With half that result for a semi-infinite interval.

[91] Note that (29) also holds at the edge from the side of the bulk, for $-\hat{a} \gg 1,[71,76]$.

[92] We thank C. Charlier for pointing out that the conjecture (29) for $V_{\ell}(r) \simeq \frac{1}{2} r^{2}+\frac{\ell^{2}}{2 r^{2}}$ is actually a consequence of Thm. 1.1 of Ref. [93] applied to $V(x) \propto x^{2}-2 \lambda \ln x$ defined in Eq. (1.7) there (scaled and shifted to satisfy the condition that the equilibrium measure is on $[-1,1])$, with $\lambda=\frac{\alpha}{\mu}$. Only the sum over $\ell$ remains to be analyzed rigorously.

[93] C. Charlier, Asymptotics of Hankel determinants with a one-cut regular potential and Fisher-Hartwig singularities, Int. Math. Res. Notices 2019, 7515 (2019).

[94] P. Calabrese, M. Mintchev, and E. Vicari, The entangle- 
ment entropy of one-dimensional systems in continuous and homogeneous space, J. Stat. Mech. P09028 (2011)

[95] Note that for $d=1$ the dependence on $R$ in (34) drops out.

[96] P. Calabrese, M. Mintchev, and E. Vicari, Entanglement entropy of one-dimensional gases, Phys. Rev. Lett. 107, 020601 (2011).

[97] H. Leschke, A. V. Sobolev, and W. Spitzer, Scaling of Rényi entanglement entropies of the free Fermi-gas ground state: a rigorous proof, Phys. Rev. Lett. 112, 160403 (2014).

[98] N. R. Smith, P. Le Doussal, S. N. Majumdar, and G. Schehr, in preparation

[99] A. Bastianello, L. Piroli, and P. Calabrese, Exact local correlations and full counting statistics for arbitrary states of the one-dimensional interacting Bose gas, Phys. Rev. Lett. 120, 190601 (2018).

[100] J. B. French, P. A. Mello, A. Pandey, Statistical Properties of Many-Particle Spectra. II. Two-Point Correlations and Fluctuations, Ann. Phys. 113, 277 (1978).

[101] E. Brézin, A. Zee, Universality of the correlations between eigenvalues of large random matrices, Nucl. Phys. B 402, 613 (1993).

[102] C. W. J. Beenakker, Universality of Brézin and Zee's spectral correlator, Nucl. Phys. B 422, 515 (1994).

[103] I. Dumitriu and A. Edelman, Matrix models for beta ensembles, J. Math. Phys. 43, 5830 (2002).

[104] P. J. Forrester and N. E. Frankel, Applications and gener- alizations of Fisher-Hartwig asymptotics, J. Math. Phys. 45, 2003 (2004).

[105] P. J. Forrester, N. E. Frankel, and T. M. Garoni, Asymptotic form of the density profile for Gaussian and Laguerre random matrix ensembles with orthogonal and symplectic symmetry, J. Math. Phys. 47, 023301 (2006)

[106] P. Vivo, S. N. Majumdar, and O. Bohigas, Large deviations of the maximum eigenvalue in Wishart random matrices, J. Phys. A: Math. Theor. 40, 4317 (2007).

[107] J. B. Hough, M. Krishnapur, Y. Peres, and B. Virág, Zeros of Gaussian analytic functions and determinantal point processes, (Vol. 51), Am. Math. Soc. (2009).

[108] N. S. Witte, and P. J. Forrester, On the variance of the index for the Gaussian unitary ensemble, Random Matrices-Theo 1, 1250010 (2012)

[109] F. Lavancier, J. Møller, and E. Rubak, Determinantal point process models and statistical inference, J. R. Stat. Soc. B 77, 853 (2014).

[110] A. Grabsch, S. N. Majumdar, G. Schehr, and C. Texier, Fluctuations of observables for free fermions in a harmonic trap at finite temperature, SciPost Phys, 4, 014 (2018).

[111] F. D. Cunden, F. Mezzadri, and N. O'Connell, Free fermions and the classical compact groups, J. Stat. Phys. 171, 768 (2018).

[112] D. S. Dean, P. Le Doussal, S. N. Majumdar, and G. Schehr, Noninteracting fermions in a trap and random matrix theory, J. Phys. A: Math. Theor. 52, 144006 (2019).

[113] There may be exceptional points when a symmetry is present, e.g. for the $\mathrm{HO}$ and $y=-x$ one has $\tilde{\phi}_{N}(x)=$ $(-1)^{N} \tilde{\phi}_{N}(y)$ and one of these term does not oscillate. However the weight of these configurations is subdominant when computing the variance.

[114] For $V(x) \neq 0$ the exact energy levels are not always dou- bly degenerate, e.g. a delta impurity at $x=0$ breaks the degeneracy between odd (sinus) and even (cosinus) wavefunctions. Note that the ground state is never degenerate. However this effect goes beyond the semiclassical approximation.

[115] Note that $\theta_{x}=\pi-\tilde{\theta}_{x}$ where $\tilde{\theta}_{x}=\pi \frac{\int_{x}^{x^{+}} d z / k_{F}(z)}{\int_{x^{-}}^{x^{+}} d z / k_{F}(z)}$ and our main formula are equivalently valid with $\theta_{x} \rightarrow \tilde{\theta}_{x}$.

[116] Although derived for $d>1$, the results (S40) and (S60) also hold for $d=1$, with $\mathcal{N}_{R}=\mathcal{N}_{[-R, R]}$, comparing with the Dyson-Mehta behavior described in Eq. (19) in the text and with (S23) respectively.

[117] For general potentials, with no known connections to RMT, one can use the generic algorithm [107] for sampling the realizations of a determinantal point process to generate the positions of the fermions see [109] and [110] (see Appendix D). 


\section{Supplementary Material for \\ Counting statistics for non-interacting fermions in a d-dimensional potential}

We give the principal details of the calculations described in the main text of the Letter.

\section{CONTENTS}

References

I. Generalities

II. Fermions in special potentials in $d=1$ and random matrix ensembles 10

III. Counting statistics and kernel in $d=1$

IV. Calculation of $K_{\mu}(x, y)^{2}$

V. More details on the results for the counting statistics in $d=1$

VI. Central potential for $d>1$ : Generalities and decoupling 16

VII. Free fermions in dimension $d \quad 17$

A. Free fermions, using the decoupling and the 1D inverse square potential 17

B. Derivation of the free fermion result from the $d$-dimensional kernel 18

VIII. General central potential in dimension $d$ and harmonic oscillator 20

IX. Edge behavior and matching with the bulk, in $d=1$ and $d>1$

X. Higher cumulants and entropy 24

A. Higher cumulants

B. Entanglement entropy 25

XI. Numerical calculations 27

\section{GENERALITIES}

Here we consider noninteracting spinless fermions in $d=1$ with single particle Hamiltonian $\hat{H}=\frac{p^{2}}{2}+V(x)$, working in units such that $\hbar=1$, and fermion mass $m=1$. For a confining potential $V(x)$, the orthonormal eigenfunctions of $\hat{H}$, denoted $\psi_{k}(x)$, are labeled by integers $k=1,2, \ldots$ such that their associated eigenenergies $\epsilon_{k}$ form an increasing sequence. The ground state wave function is the Slater determinant $\Psi_{0}\left(x_{1}, \ldots, x_{N}\right)=\frac{1}{\sqrt{N}} \operatorname{det}_{1 \leq i, j \leq N} \psi_{j}\left(x_{i}\right)$, and the joint probability distribution function (JPDF) of the fermion positions takes a determinantal form, $\left|\Psi_{0}\left(x_{1}, \ldots, x_{N}\right)\right|^{2}=$ $\frac{1}{N !} \operatorname{det}_{1 \leq i, j \leq N} K_{\mu}\left(x_{i}, x_{j}\right)$ in terms of the kernel $K_{\mu}$ [11]

$$
K_{\mu}(x, y)=\sum_{k} \theta\left(\mu-\epsilon_{k}\right) \psi_{k}^{*}(x) \psi_{k}(y)=\sum_{k=1}^{N} \psi_{k}^{*}(x) \psi_{k}(y) .
$$

Here $\mu$ is the Fermi energy, related to $N$ via $N=\sum_{k} \theta\left(\mu-\epsilon_{k}\right)$. Using the orthonormalization of the eigenfunctions $\int \psi_{k}^{*}(x) \psi_{k^{\prime}}(x)=\delta_{k, k^{\prime}}$, it is straightforward to show that the kernel satisfies

$$
\int K_{\mu}(x, z) K_{\mu}(z, y) d z=K_{\mu}(x, y),
$$


a useful property which is called the "reproducibility" of the kernel. In particular, it implies [14] that each $n \leq N$ point correlation function of the fermion positions also take a determinantal form in terms of $K_{\mu}$ (see below for $n=2$ ), in particular the mean fermion density (normalized to $\left.\int d x \rho(x)=N\right)$ is $\rho(x)=K_{\mu}(x, x)$.

These properties extend to the case of a non confining potential $V(x)$, with a continuum spectrum, e.g. free fermions $V(x)=0$, with the kernel given by a continuum limit of (S1). In this case $N$ can be infinite and the control parameter is $\mu$. This is extended to $d>1$ starting from Section VI.

\section{FERMIONS IN SPECIAL POTENTIALS IN $d=1$ AND RANDOM MATRIX ENSEMBLES}

As mentioned in the text, for specific potentials $V(x)$ and geometries, the JPDF of the fermion positions $\vec{x}=\left\{x_{i}\right\}$ in the ground state can be mapped, for any $N$, to the JPDF of the eigenvalues $\vec{\lambda}=\left\{\lambda_{i}\right\}$ of some random matrices.

- Free fermions on a circle of perimeter $L$ with $V(x)=0$, map to the eigenvalues $\lambda_{j}=e^{i 2 \pi x_{j} / L}$ of random matrices from the circular unitary ensemble (CUE) $[14,23,78,111]$. The eigenfunctions are plane waves $\sim e^{2 \pi i p x / L}$, $p=0, \pm 1, \ldots$, and $\left|\Psi_{0}(\vec{x})\right|^{2} \propto P_{\mathrm{CUE}}(\vec{\lambda}) \propto\left|\Delta_{N}(\lambda)\right|^{\beta}$, where $\Delta_{N}(z)=\prod_{1 \leq i<j \leq N}\left(z_{i}-z_{j}\right)$ and $\beta=2$. The mean density is uniform $\rho(x)=k_{F} / \pi=\frac{N}{L}$ with $k_{F}=\sqrt{2 \mu}$.

- Fermions on $\mathbb{R}$ in the harmonic oscillator $(\mathrm{HO})$ potential, $V(x)=\frac{x^{2}}{2}$, map to the eigenvalues $\lambda_{i}=x_{i}$ of Hermitian random matrices from the Gaussian unitary ensemble (GUE) [10, 11, 20, 112]. The eigenfunctions are $\sim e^{-x^{2} / 2} H_{n}(x), n=0,1, \ldots$, where $H_{n}$ are the Hermite polynomials, with energies (with the labeling used here) $\epsilon_{n+1}=n+\frac{1}{2}$, and $\left|\Psi_{0}(\vec{x})\right|^{2}=P_{\mathrm{GUE}}(\vec{\lambda}) \propto|\Delta(\lambda)|^{\beta} e^{-\sum_{i} \lambda_{i}^{2}}$ with $\beta=2$. For large $N$, the mean density is the semi-circle of support $\left[x^{-}, x^{+}\right]$with $x^{ \pm} \simeq \pm \sqrt{2 N}$, i.e., $\rho(x) \simeq \rho^{\text {bulk }}(x)=k_{F}(x) / \pi$, where $k_{F}(x)=\sqrt{\left(2 \mu-x^{2}\right)_{+}}$ and $\mu \simeq N$. We denote here and below $(x)_{+}=\max (x, 0)$.

- Fermions on $\mathbb{R}^{+}$in the inverse square potential, $V(x)=\frac{x^{2}}{2}+\frac{\alpha(\alpha-1)}{2 x^{2}}, \alpha \geq 1 / 2$, map to eigenvalues $\lambda_{i}=x_{i}^{2}$ of Wishart-Laguerre random matrices [79-81, 112]. The eigenfunctions are $\sim x^{\alpha} L_{n}^{\alpha-1 / 2}\left(x^{2}\right) e^{-x^{2} / 2}$, where $L_{n}^{\gamma}(z)$ are the generalized Laguerre polynomials, with energies $\epsilon_{n}=2 n+\alpha+\frac{1}{2}, n=0,1, \ldots$ One has $\left|\Psi_{0}(\vec{x})\right|^{2} d^{N} \vec{x} \propto \prod_{i=1}^{N} x_{i}^{2 \alpha} e^{-x_{i}^{2}} d x_{i} \prod_{1 \leq j, k \leq N}\left(x_{j}^{2}-x_{k}^{2}\right)^{2} \propto P_{\mathrm{LUE}}(\vec{\lambda}) d^{N} \vec{\lambda}$ where $P_{\mathrm{LUE}}(\vec{\lambda}) \propto$ $e^{-\frac{\beta}{2} \sum_{i=1}^{N} \lambda_{i}} \prod_{i=1}^{N} \lambda_{i}^{\frac{\beta}{2}(M-N+1)-1}\left|\Delta_{N}(\vec{\lambda})\right|^{\beta}$ with $\beta=2$ is the JPDF of the eigenvalues $\lambda_{j}$ of the Laguerre-Wishart complex random matrices (also called LUE) of the form $X^{T} X$ where $X$ is a $M \times N$ rectangular random matrix with i.i.d. unit complex Gaussian entries with $M \geq N$ (see [106] for the case $M<N$ ) and $\alpha-\frac{1}{2}=M-N$. The mean fermion density is $\rho^{\text {bulk }}(x)=\frac{1}{\pi} \sqrt{\left(2 \mu-x^{2}-\frac{\alpha(\alpha-1)}{x^{2}}\right)}$, which maps to the Marcenko-Pastur density for the $\lambda_{i}$. The case $\alpha=0$ corresponds to the half-harmonic oscillator with a half-semi-circle density for the fermions $x_{i}$. In the other limiting case, $V(x)=\frac{\alpha(\alpha-1)}{2 x^{2}}$, the spectrum of $\hat{H}$ is continuous and the fermions are described by the Bessel kernel (see below and [81]).

- Fermions in the "Jacobi box" with $x \in[0, L]$ and potential $V(x)=\frac{\pi^{2}}{L^{2}}\left(\frac{\mathrm{a}^{2}-\frac{1}{4}}{8 \sin ^{2}(\pi x / 2 L)}+\frac{\mathrm{b}^{2}-\frac{1}{4}}{8 \cos ^{2}(\pi x / 2 L)}\right)$, map to the eigenvalues $\lambda_{i}=\frac{1-\cos \left(\pi x_{i} / L\right)}{2}$ of the Jacobi unitary ensemble (JUE) of RMT. The eigenvectors can be expressed in terms of Jacobi polynomials and the energies are $\epsilon_{n}=\frac{\pi^{2}}{2 L^{2}}\left(n+\frac{\mathrm{a}+\mathrm{b}+1}{2}\right)^{2}$. One has $\left|\Psi_{0}(\vec{x})\right|^{2} d^{N} \vec{x}=P_{J}(\vec{\lambda}) d^{N} \lambda$ where $P_{J}(\vec{\lambda}) \propto \prod_{i} \lambda_{i}^{\mathrm{a}}\left(1-\lambda_{i}\right)^{\mathrm{b}} \Delta_{N}(\vec{\lambda})$. For more details see Appendix $\mathrm{C}$ in [81]. In the case $\mathrm{a}=\mathrm{b}=\frac{1}{2}$ one obtains the hard box with $V(x)=0$ and Dirichlet boundary conditions at $x=0, L$ (note that the eigenfunctions vanish at $x=0, L$ for any $\mathrm{a}, \mathrm{b})$.

\section{COUNTING STATISTICS AND KERNEL IN $d=1$}

For $N$ fermions on the infinite line one defines the height field observable $h(a)=\int_{-\infty}^{a} \hat{\rho}(x) d x=\mathcal{N}_{]-\infty, a]}=$ $N-\mathcal{N}_{[a,+\infty}$, with $\hat{\rho}(x)=\sum_{i=1}^{N} \delta\left(x-x_{i}\right)$, where $\mathcal{N}_{\mathcal{D}}$ denotes the number of fermions in the subset $\mathcal{D}$. The number of fermions in the interval $[a, b]$ is thus $\mathcal{N}_{[a, b]}=h(b)-h(a)$, and its quantum average is $\left\langle\mathcal{N}_{[a, b]}\right\rangle=\int_{a}^{b} \rho(x) d x$ where $\rho(x)=\langle\hat{\rho}(x)\rangle$ is the mean density and here $\langle\ldots\rangle$ denotes averages w.r.t. the ground state quantum JPDF $\left|\Psi_{0}\right|^{2}$.

Next one defines the two point covariance of the height field, i.e., the function $H(x, y)=\operatorname{Cov}(h(x), h(y))=$ $\langle h(x) h(y)\rangle-\langle h(x)\rangle\langle h(y)\rangle$. If $H(x, y)$ is known, then the variance of the number of fermions in any interval, or any 
collection of intervals is also known as

$$
\begin{aligned}
& \operatorname{Var} \mathcal{N}_{[a, b]}=H(a, a)+H(b, b)-2 H(a, b) \quad, \quad \operatorname{Var} \mathcal{N}_{]-\infty, a]}=\operatorname{Var} \mathcal{N}_{[a,+\infty[}=H(a, a), \\
& \operatorname{Var} \mathcal{N}_{\cup_{i}\left[a_{i}, b_{i}\right]}=\sum_{i j} H\left(a_{i}, a_{j}\right)+H\left(b_{i}, b_{j}\right)-2 H\left(a_{i}, b_{j}\right) .
\end{aligned}
$$

The height field covariance is related to the two point correlation function defined as

$$
R_{2}(x, y):=\left\langle\sum_{1 \leq i \neq j \leq N} \delta\left(x-x_{i}\right) \delta\left(y-x_{j}\right)\right\rangle=\langle\hat{\rho}(x) \hat{\rho}(y)\rangle-\delta(x-y)\langle\hat{\rho}(x)\rangle .
$$

Indeed, from its definition one has $\partial_{x} \partial_{y} H(x, y)=\langle\hat{\rho}(x) \hat{\rho}(y)\rangle-\rho(x) \rho(y)=R_{2}(x, y)+\delta(x-y) \rho(x)-\rho(x) \rho(y)$, using (S5). For noninteracting fermions the correlation functions are given by determinants $[11,14]$ for instance

$$
R_{2}(x, y)=N(N-1) \int_{-\infty}^{+\infty} d x_{3} \ldots d x_{N}\left|\Psi_{0}\left(x, y, x_{3}, \ldots, x_{N}\right)\right|^{2}=K_{\mu}(x, x) K_{\mu}(y, y)-K_{\mu}(x, y)^{2}
$$

where we used that $K_{\mu}$ is symmetric in its arguments in the cases of interest here. Eq. $(\mathrm{S} 6)$ then implies $\partial_{x} \partial_{y} H(x, y)=$ $\delta(x-y) \rho(x)-K_{\mu}(x, y)^{2}$, which is the equation (11) of the text. In the text we provide solutions to this equation, but one can equivalently compute the height covariance from the kernel by integrating (11) of the main text twice which yields

$$
H(a, b)=\int_{-\infty}^{a} d x \int_{-\infty}^{b} d y\left(-K_{\mu}(x, y)^{2}+\rho(x) \delta(x-y)\right)=\int_{-\infty}^{\min (a, b)} d x \int_{\max (a, b)}^{+\infty} d y K_{\mu}(x, y)^{2}
$$

where in the second equality we used that $\rho(x)=K_{\mu}(x, x)=\int_{-\infty}^{+\infty} d y K_{\mu}(x, y)^{2}$, which follows from the reproducibility (S2) of the kernel.

For fermions on $\mathbb{R}^{+}$, e.g. for the inverse square well, one should replace everywhere above $-\infty$ by 0 . More generally, for fermions defined in an interval $\left[c^{-}, c^{+}\right]$one should replace everywhere above $-\infty$ by $c^{-}$and $+\infty$ by $c^{+}$, e.g. for the Jacobi box $c^{-}=0$ and $c^{+}=L$. In all cases we call for convenience below $\left[c^{-}, a\right]$ and $\left[a, c^{+}\right]$a "semi-infinite" interval.

\section{CALCULATION OF $K_{\mu}(x, y)^{2}$}

In this section we provide more details on the calculation of the kernel $K_{\mu}(x, y)$ using the WKB asymptotics, leading to (13) in the main text, as well as the evaluation of $K_{\mu}(x, y)^{2}$ on macroscopic scales given in (15) in the text, later used to obtain the result (16) in the text for the height correlator. We discuss separately the case of a confining potential presented in the text, and the case of fermions on the circle.

Fermions in a confining potential. In the large $N$ limit the sum over $k$ in (S1) is dominated by $k \gg 1$, i.e., semi-classical eigenstates. Consider first a confining potential $V(x)$ such that there are exactly two turning points at the Fermi energy, i.e., two roots to $V\left(x^{ \pm}\right)=\mu$. Energy levels are then non-degenerate. The semi-classical eigenstates obey the quantization condition $\int d z \sqrt{2\left(\epsilon_{k}-V(z)\right)_{+}} \simeq \pi k$. This leads to $d \epsilon_{k} / d k=\left(\frac{1}{\pi} \int \frac{d z}{\sqrt{2\left(\epsilon_{k}-V(z)\right)_{+}}}\right)^{-1}$, where here and below we introduce the standard convention that $1 /(x)_{+}^{\gamma}=0$ for $x<0$ and $1 /(x)_{+}^{\gamma}=1 / x^{\gamma}$ for $x>0$ (used below for the values $\gamma=1 / 2$ and $\gamma=1 / 4)$. For $k=N, \epsilon_{N}=\mu$, this leads to $d N / d \mu=\int_{x^{-}}^{x^{+}} d z /\left(\pi k_{F}(z)\right)$. For $x$ in the bulk we can use the WKB asymptotics $[64,65]$

$$
\psi_{k}(x) \simeq \frac{C_{k}}{\left[2\left(\epsilon_{k}-V(x)\right)_{+}\right]^{1 / 4}} \sin \left(\phi_{k}(x)+\frac{\pi}{4}\right)
$$

where $\phi_{k}(x)=\int_{-\infty}^{x} d z \sqrt{2\left(\epsilon_{k}-V(z)\right)_{+}}$is a fast oscillating function. The normalization is $C_{k}^{2}=\frac{2}{\pi} \frac{d \epsilon_{k}}{d k}$ [66]. It naturally allows to recover the usual formula for the bulk density as

$$
\rho(x)=\sum_{k=1}^{N}\left|\psi_{N}(x)\right|^{2} \simeq \frac{1}{\pi} \int_{0}^{\mu} \frac{d \epsilon}{\sqrt{2(\epsilon-V(x))_{+}}}=\frac{k_{F}(x)}{\pi} \quad, \quad k_{F}(x)=\sqrt{2(\mu-V(x))_{+}}
$$


where we have replaced $\sum_{k} \frac{d \epsilon_{k}}{d k} \simeq \int d \epsilon$ and $\sin ^{2} \rightarrow 1 / 2$ up to fast oscillating terms which are neglected. Note that (S8) is approximated by zero in the classically forbidden region, $\epsilon_{k}<V(x)$, which was essential to recover the bulk mean density in (S9). Eq. (S8) thus does not accurately describe terms in the sum (S1) such that $\epsilon_{k} \simeq V(x)$, but for $x$ in the bulk it is safe to assume that these contributions are subdominant (as they are in (S9)). Note also that we do not need to assume that there are also only two roots to $V\left(x^{ \pm}(\epsilon)\right)=\epsilon$ for all $\epsilon<\mu$, the formula below are also correct, to the same order, for e.g. a double well potential (whenever $\mu>V_{0}$ where $V_{0}$ is the local maximum).

Below we need the limit of large $N$ at fixed $m$, i.e., an expansion near the Fermi energy, for which one can write,

$$
\phi_{N-m}(x)=\int_{x^{-}}^{x} d z \sqrt{2\left(\epsilon_{N-m}-V(z)\right)_{+}}=\phi_{N}(x)-m \frac{d \epsilon_{N}}{d N} \int_{x^{-}}^{x} \frac{d z}{\sqrt{2\left(\epsilon_{N}-V(z)\right)_{+}}}+o(1)=\phi_{N}(x)-m \theta_{x}+o(1)
$$

where, as in the text, we define

$$
\theta_{x}=\frac{d \mu}{d N} \int_{x^{-}}^{x} \frac{d z}{k_{F}(z)}=\pi \frac{\int_{x^{-}}^{x} \frac{d z}{k_{F}(z)}}{\int_{x^{-}}^{x^{+}} \frac{d z}{k_{F}(z)}} .
$$

As an example, for the HO one has $\epsilon_{k}=k-\frac{1}{2}$ and $\phi_{N}(x)=\frac{1}{2} x \sqrt{2 \epsilon_{N}-x^{2}}+\epsilon_{N}\left(\arcsin \left(x / \sqrt{2 \epsilon_{N}}\right)+\frac{\pi}{2}\right)$, and $\theta_{x}=\arccos (-x / \sqrt{2 \mu})$ with $\mu \simeq N$. We can compare the formulae (S8) and (S10) with the Plancherel-Rotach formula as given in [105] Eq. (3.10), with $k=N-m, n=k-1$, setting $x=\sqrt{2 N} X$, for large $N$ and $m$ fixed

$$
\begin{aligned}
\psi_{k=N-m} & (x=\sqrt{2 N} X)=\left(\frac{1}{\sqrt{\pi} 2^{n} n !}\right)^{1 / 2} e^{-x^{2} / 2} H_{n}(x) \\
= & 2^{1 / 4} \sqrt{\frac{1}{\pi}} \frac{1}{\left(1-X^{2}\right)^{1 / 4}} N^{-\frac{1}{4}-\frac{m+1}{2}} \frac{(N !)^{1 / 2}}{((N-m-1) !)^{1 / 2}} \cos \left(\Phi_{N}(X)+(m+1) \arccos X\right)\left(1+O\left(\frac{1}{N}\right)\right)
\end{aligned}
$$

with $\Phi_{N}(X)=N X \sqrt{1-X^{2}}+\left(N+\frac{1}{2}\right) \arcsin X-\frac{N \pi}{2}$. Using in (S8) that $\frac{C_{N-m}}{\left(\left(2 \epsilon_{N-m}-x^{2}\right)_{+}\right)^{1 / 4}} \simeq \frac{C_{N}}{\left(\left(2 \epsilon_{N}-x^{2}\right)_{+}\right)^{1 / 4}}$ and $d \epsilon_{N} / d N=1$ we see that the prefactors of the oscillating term agree in formulas (S8) and (S12). In addition one can check that

$$
\Phi_{N}(X)+(m+1) \arccos X=\phi_{N}(x)-m \arccos (-X)+\frac{3 \pi}{4}-(N-m) \pi+o(1)
$$

hence

$$
\cos \left(\Phi_{N}(X)+(m+1) \arccos X\right) \simeq(-1)^{N-m+1} \sin \left(\phi_{N}(x)-m \arccos (-X)+\frac{\pi}{4}\right)
$$

for $x=\sqrt{2 N} X$. Hence up to a factor $(-1)^{k+1}$, which has no effect in our calculation below, the Plancherel-Rotach formula as given in [105] coincides with the WKB approximation (S8), together with our estimate (S10).

We can now insert the WKB asymptotics (S8)-(S10) for the eigenstates into the formula for the kernel (S1). We will see that, in the limit of large $N$ and for the observable of interest, the sum over $k$ is dominated by $k=N-m$ with $m=O(1)$. Hence we can use the same approximations as in the previous paragraph, and take for the WKB eigenstates the form $\psi_{N-m}(x) \simeq \frac{C_{N}}{(2(\mu-V(x)))^{1 / 4}} \cos \left(\phi_{N}(x)-m \theta_{x}-\frac{\pi}{4}\right)$, recalling that $\epsilon_{N}=\mu$. Using that $C_{N}^{2}=\frac{2}{\pi} \frac{d \mu}{d N}$ and the identity $\cos a \cos b=\frac{1}{2}(\cos (a+b)+\cos (a-b))$ we obtain

$$
K_{N}(x, y) \simeq \frac{d \mu / d N}{\pi \sqrt{k_{F}(x) k_{F}(y)}} \sum_{m \geq 0} \sum_{\sigma= \pm 1} \cos \left(\phi_{N}(x)-\sigma \phi_{N}(y)-m\left(\theta_{x}-\sigma \theta_{y}\right)-\frac{\pi}{4}(1-\sigma)\right) .
$$

Now we perform the geometric sum over $m$, i.e., we write

$$
\sum_{m \geq 0} \cos (A-m B)=\operatorname{Re} \sum_{m \geq 0} e^{i A-i m B}=\operatorname{Re} \frac{e^{i A}}{1-e^{-i B}}=\frac{\sin \left(A+\frac{B}{2}\right)}{2 \sin \frac{B}{2}}
$$

with $A=\phi_{N}(x)-\sigma \phi_{N}(y)-\frac{\pi}{4}(1-\sigma)$ and $B=\theta_{x}-\sigma \theta_{y}$ and we obtain the Eq. (13) of the main text with $\tilde{\phi}_{N}(x)=\phi_{N}(x)+\frac{1}{2} \theta_{x}-\frac{\pi}{4}$.

We have checked numerically, in the case of the harmonic oscillator, that (13) of the main text provides an excellent approximation not only of the amplitude but also of the phase of the (rapid) oscillations. Note that in that case (and 
more generally for the potentials related to RMT) one can use the equivalent Christoffel-Darboux form of the kernel $[11,14,78]$ (a consequence of the recurrence relations of the Hermite polynomials)

$$
K_{\mu}(x, y)=\sqrt{\frac{N}{2}} \frac{\psi_{N+1}(x) \psi_{N}(y)-\psi_{N}(x) \psi_{N+1}(y)}{x-y}=\frac{e^{-\left(x^{2}+y^{2}\right) / 2}}{\sqrt{\pi} 2^{N}(N-1) !} \frac{H_{N}(x) H_{N-1}(y)-H_{N-1}(x) H_{N}(y)}{x-y}
$$

with our conventions (see (S12) and above). Using the Plancherel-Rotach formula (S12) one then arrives at the same result (13) of the main text. Since that method circumvents the summation over the eigenstates, it provides an independent check of our results in some special cases.

The next step is to calculate $K_{\mu}(x, y)^{2}$ when the distance $|x-y|$ is macroscopic. The direct square of (13) in the main text leads to the sum of two parts. The first part is obtained from the $\sin ^{2}\left(\tilde{\phi}_{N}(x)-\sigma \tilde{\phi}_{N}(y)\right)$ terms and the replacement of each of them by $1 / 2$ (ii) the second part is a linear combination of terms proportional to $\cos 2\left(\tilde{\phi}_{N}(x)-\sigma \tilde{\phi}_{N}(y)\right)$ and (from the product of the sine) $\cos 2 \tilde{\phi}_{N}(x)$ and $\cos 2 \tilde{\phi}_{N}(y)$. These terms oscillate on microscopic scales $O\left(1 / k_{F}(x), 1 / k_{F}(y)\right)$, hence any local average of them on macroscopic scales (e.g. upon integration over $x, y$ when computing the height correlator in (S7)) will give negligible contributions [113]. Retaining thus only the first part, and using that $\sum_{\sigma= \pm 1} \frac{1}{\sin ^{2}\left(\frac{\theta_{x}-\sigma \theta_{y}}{2}\right)}=4 \frac{1-\cos \left(\theta_{x}\right) \cos \left(\theta_{y}\right)}{\left(\cos \theta_{x}-\cos \theta_{y}\right)^{2}}$ we obtain Eq. (15) of the text. Note that we have performed a numerical check of the formula for $K_{\mu}^{2}$ for the HO in Fig. 7 in Section XI.

Fermions on the circle. Consider now fermions on the circle $x \in[0, L]$, and a periodic potential of period $L$ such that $V(x)<\mu$ for all $x$, i.e., without turning points. Let us start with the case $V(x)=0$ (i.e., the CUE) which is quite pedagogical. The kernel reads $K_{\mu}(x, y)=\frac{1}{L} \sum_{p=-p_{N}}^{p_{N}} e^{\frac{2 \pi i p(x-y)}{L}}=\frac{\sin \left(k_{F}(x-y)\right)}{L \sin \pi \frac{x-y}{L}}$ with $k_{F}=\frac{N \pi}{L}$ and $p_{N}=\frac{N-1}{2}$ (we restrict ourselves here to the case where $N$ is odd. In this case the many-body ground state is not degenerate). For $x-y \ll L, K_{\mu}(x, y)$ reduces to the sine-kernel. For $x-y=O(L)$ one has, discarding the fast oscillating term $\cos \left(2 k_{F}(x-y)\right)$

$$
K_{\mu}(x, y)^{2} \simeq \frac{1}{2 L^{2} \sin ^{2}\left(\pi \frac{x-y}{L}\right)}=\frac{1}{8 \pi^{2}} \frac{d \theta_{x}}{d x} \frac{d \theta_{y}}{d y} \frac{1}{\sin ^{2} \frac{1}{2}\left(\theta_{y}-\theta_{x}\right)}=\partial_{x} \partial_{y} \frac{1}{2 \pi^{2}} \log \left|\sin \frac{\theta_{x}-\theta_{y}}{2}\right|
$$

with $\theta_{x}=2 \pi x / L$ for free fermions. We now show that the last two identities extend to a general potential $V(x)$ where $\theta_{x}$ is given below (by a different formula than the one for the confining well).

In the semi-classical approximation one can consider that the energy levels $\epsilon_{k}>\max _{x} V(x)$ are doubly degenerate on the circle [114]. The WKB states are $\psi_{k}^{ \pm}(x) \simeq \frac{C_{k}}{\left[2\left(\epsilon_{k}-V(x)\right)\right]^{1 / 4}} e^{ \pm i \phi_{k}(x)}$ with $\phi_{k}(x)=\int_{0}^{x} d u \sqrt{2\left(\epsilon_{k}-V(u)\right)}$. Their normalization implies $C_{k}^{2}=\frac{1}{2 \pi} \frac{d \epsilon_{k}}{d k}$, using the quantization condition $\int_{0}^{L} d x \sqrt{2\left(\epsilon_{k}-V(x)\right)}=2 k \pi$. Denoting $n$ the highest fully occupied level, $\mu=\epsilon_{n}$, we have $N=2 n+1 \simeq 2 n$ for $N \gg 1$, and $\int_{0}^{L} d x \sqrt{2(\mu-V(x))} \simeq 2 n \pi \simeq$ $N \pi$ so we have $\rho(x) \simeq k_{F}(x) / \pi$ as usual and $d N / d \mu \simeq \int_{0}^{L} \frac{d x}{\pi k_{F}(x)}$. In particular $C_{n}^{2}=\frac{1}{2 \pi} \frac{d \epsilon_{n}}{d n} \simeq \frac{1}{\pi} \frac{d \mu}{d N}$, with a factor of 2 compared to the case of two turning points. Inserting the WKB wavefunctions in the kernel $K_{\mu}(x, y) \simeq$ $\sum_{\sigma= \pm 1} \sum_{m=0}^{n} \psi_{n-m}^{\sigma}(x)^{*} \psi_{n-m}^{\sigma}(y)$ one expands $\phi_{n-m}(x)=\phi_{n}(x)-m \theta_{x}+o(1)$, where $\theta_{x}=\frac{d \phi_{n}(x)}{d n}=\frac{d \epsilon_{n}}{d n} \int_{0}^{x} \frac{d u}{\sqrt{2(\mu-V(u))}}$.

Since $\frac{d \epsilon_{n}}{d n} \simeq 2 \frac{d \mu}{d N}$, one thus obtains that for the circle $\theta_{x}=2 \pi \frac{\int_{0}^{x} \frac{d z}{k_{F}(z)}}{\int_{0}^{L} \frac{d z}{k_{F}(z)}}$. Performing the same manipulations as in the text we obtain

$$
K_{\mu}(x, y) \simeq \frac{2}{\pi} \frac{d \mu / d N}{\sqrt{k_{F}(x) k_{F}(y)}} \sum_{m=0}^{n} \cos \left(\phi_{n}(y)-\phi_{n}(x)+m\left(\theta_{x}-\theta_{y}\right)\right) \simeq \frac{1}{\pi} \frac{d \mu / d N}{\sqrt{k_{F}(x) k_{F}(y)}} \frac{\sin \left(\tilde{\phi}_{n}(y)-\tilde{\phi}_{n}(x)\right)}{\sin \frac{1}{2}\left(\theta_{y}-\theta_{x}\right)}
$$

where $\phi_{n}(x)=\int_{0}^{x} d u \sqrt{2(\mu-V(u))}$ and $\tilde{\phi}_{n}(x)=\phi_{n}(x)+\frac{1}{2} \theta_{x}$. Using that $d \theta_{x}=2 \frac{d \mu}{d N} \frac{d x}{k_{F}(x)}$ and $\sin ^{2} \rightarrow 1 / 2$ up to fast oscillating terms we arrive at (S18).

\section{MORE DETAILS ON THE RESULTS FOR THE COUNTING STATISTICS IN $d=1$}

Consider fermions with Fermi energy $\mu$ in a general potential $V(x)$ in $d=1$, defined on the interval $\left[c^{-}, c^{+}\right]$(which may be infinite or semi-infinite). In this section we explain the formula for the variance of the number of fermions $\mathcal{N}_{\mathcal{D}}$ in a macroscopic interval in the large $N, \mu$ limit. These formula will differ slightly depending on whether the 
bulk density, $\rho(x)=k_{F}(x) / \pi$, has e.g. (i) a bounded support on a single interval $\left[x^{-}, x^{+}\right]$with $V\left(x^{ \pm}\right)=\mu$, (ii) a semi-infinite support $\left[x^{-},+\infty\right.$ [ (iii) no edge such as fermions on the circle with $V(x)<\mu$ for all $x$. Other cases, such as multiple interval supports can also be studied.

(i) Confining potentials: two turning points. The case (i) relevant for a confining trap was detailed in the main text, leading to formula (2) in the text for the variance of $\mathcal{N}_{[a, b]}$ and formula (16), (20) in the text for the height field correlator. They are expressed in terms of the semi-classical variable $\theta_{x}$ defined in (3) in the text, which reaches values 0 and $\pi$ at $x^{-}$and $x^{+}$, and has the interpretation of the time along the classical trajectories (normalized by the period). This case corresponds to two turning points at energy $\mu$ at positions $x^{-}$and $x^{+}$. For the potentials $V(x)$ related to RMT introduced in Section II, $\theta_{x}$ has a simple expression. One finds, from the definition in (3) in the text

$$
\theta_{x}= \begin{cases}\arccos \left(\frac{-x}{\sqrt{2 \mu}}\right), \quad V(x)=\frac{1}{2} x^{2} \quad, \quad x^{ \pm}= \pm \sqrt{2 \mu} \\ \arccos \left(\frac{\mu-x^{2}}{\sqrt{\mu^{2}-\alpha(\alpha-1)}}\right), & V(x)=\frac{x^{2}}{2}+\frac{\alpha(\alpha-1)}{2 x^{2}} \quad, \quad\left(x^{ \pm}\right)^{2}=\mu \pm \sqrt{\mu^{2}-\alpha(\alpha-1)} \\ \arccos \left(\frac{\cos (\pi x / L)-A}{B}\right), & V(x)=\frac{\pi^{2}}{L^{2}}\left(\frac{a^{2}-\frac{1}{4}}{8 \sin ^{2}(\pi x / 2 L)}+\frac{b^{2}-\frac{1}{4}}{8 \cos ^{2}(\pi x / 2 L)}\right) \quad, \quad \cos \left(\pi x_{ \pm} / L\right)=A \mp B\end{cases}
$$

with $A=\frac{\mathrm{b}^{2}-\mathrm{a}^{2}}{8 \mu}$ and $B=\sqrt{1+A^{2}-\frac{1-2 \mathrm{a}^{2}-2 \mathrm{~b}^{2}}{8 \mu}}$. We now discuss each potential separately. The following formula are useful in all cases [115]

$$
\frac{d \mu}{d N} \simeq \frac{\pi}{\int_{x^{-}}^{x^{+}} d z / k_{F}(z)}, \theta_{x} \simeq \frac{d \mu}{d N} \int_{x_{-}}^{x} d z / k_{F}(z) \quad, \quad 2\left(\sin \frac{1}{2}(\arccos p \pm \arccos q)\right)^{2}=1-p q \pm \sqrt{\left(1-p^{2}\right)\left(1-q^{2}\right)} .
$$

- For the HO (first line in (S20)) one has $d \mu / d N \simeq 1$ and from (S20) $\left|\sin \theta_{a}\right|=\sqrt{1-\frac{a^{2}}{2 \mu}}=\frac{k_{F}(a)}{\sqrt{2 \mu}}$. Inserting in (20) in the text it leads to the explicit expression for the variance for the semi-infinite interval

$$
\operatorname{Var} \mathcal{N}_{[a,+\infty[}=\operatorname{Var} \mathcal{N}_{]-\infty, a]}=\frac{1}{2 \pi^{2}}\left(\log \mu+\frac{3}{2} \log \left(1-\tilde{a}^{2}\right)+c_{2}+2 \log 2+o(1)\right) .
$$

Using in addition the trigonometric relation (S21) one can check that the general formula (2) in the text leads to the expression

$$
2 \pi^{2} \operatorname{Var} \mathcal{N}_{[a, b]}=2 \log \mu+\frac{3}{2} \log \left[\left(1-\tilde{a}^{2}\right)\left(1-\tilde{b}^{2}\right)\right]+2 \log \left|\frac{4|\tilde{a}-\tilde{b}|}{1-\tilde{a} \tilde{b}+\sqrt{\left(1-\tilde{a}^{2}\right)\left(1-\tilde{b}^{2}\right)}}\right|+2 c_{2}+o(1)
$$

in the limit $\mu \rightarrow+\infty$ with $\tilde{a}=\frac{a}{\sqrt{2 \mu}}, \tilde{b}=\frac{b}{\sqrt{2 \mu}}$ fixed, $-1<\tilde{a} \neq \tilde{b}<1$. For $a=-b$ the leading-order term in (S23) agrees with the Coulomb gas calculations in $[20,21]$. The $O(1)$ term also agrees with some exact results by other methods in the RMT context: 1) Eq. (S22) for $a=0$ agrees with the calculation of the "index" in [15, 16], see also [108] where higher order corrections in $1 / N$ where obtained using Painlevé equations. In particular the leading corrections to (S22) are $\left.O\left(\frac{\log N}{N}\right) ; 2\right)$ a study of Fisher-Hartwig asymptotics using Riemann Hilbert methods in [58] (for the comparison with this work see Section X A). Our results (S23) and (S22) are compared with numerical simulations in Fig. 4 in Section XI.

- For the inverse square well (second line in (S20)) one has $d \mu / d N \simeq 2$ and from (S20), $\left|\sin \theta_{a}\right|=\frac{a k_{F}(a)}{\sqrt{\mu^{2}-\alpha(\alpha-1)}}$. Formula (20) in the text then leads to

$$
\operatorname{Var} N_{[0, a]}=\operatorname{Var} N_{[a,+\infty[}=\frac{1}{2 \pi^{2}}\left[\log \left(\frac{a k_{F}(a)^{3}}{\sqrt{\mu^{2}-\alpha(\alpha-1)}}\right)+c_{2}\right]+o(1)
$$

which leads to the equation (21) in the text with $\lambda^{2}=\frac{\alpha(\alpha-1)}{\mu^{2}}$ and $\tilde{a}=a / \sqrt{2 \mu}$. In addition, using the above relations we find that (2) in the text leads to

$$
\left(2 \pi^{2}\right) \operatorname{Var} N_{[a, b]}=2 \log \mu+\log \left(16 \tilde{a} \tilde{b} \kappa_{\tilde{a}}^{3} \kappa_{\tilde{b}}^{3}\right)+2 \log \frac{\left|\tilde{a}^{2}-\tilde{b}^{2}\right|}{\tilde{a}^{2}+\tilde{b}^{2}-2 \tilde{a}^{2} \tilde{b}^{2}-\frac{\lambda^{2}}{2}+2 \tilde{a} \tilde{b} \kappa_{\tilde{a}} \kappa_{\tilde{b}}}+2 c_{2}+o(1)
$$

with $\kappa_{\tilde{a}}=\left(1-\tilde{a}^{2}-\frac{\lambda^{2}}{4 \tilde{a}^{2}}\right)^{1 / 2}$ and $\kappa_{\tilde{b}}=\left(1-\tilde{b}^{2}-\frac{\lambda^{2}}{4 \tilde{b}^{2}}\right)^{1 / 2}$. Eq. (S25) is obtained in the limit of large $\mu$ with $\tilde{a}, \tilde{b}$ fixed and $\lambda=O(1)$ fixed, i.e., $\alpha \sim \mu$. For $\lambda=0$ our result (S25) agrees with the Fisher-Hartwig asymptotics 
obtained using Riemann Hilbert methods for the LUE in [89], which assumes $\alpha=O(1)$ (see also Section $\mathrm{XA}$ ). Finally, one can check that as $\tilde{a}-\tilde{b} \ll 1$, Eq. (S25) reproduces the result for a microscopic interval, $\pi^{2} \operatorname{Var} \mathcal{N}_{[a, b]}=\log \left(2 \mu|\tilde{a}-\tilde{b}| \kappa_{\tilde{a}}\right)+c_{2}=\log \left(|a-b| k_{F}(a)\right)+c_{2}$. The result for $\operatorname{Var} \mathcal{N}_{[0, a]}$ is compared with numerical simulations in Fig. 5 in Section XI.

- For the Jacobi box (third line in (S20)), one can check directly that $\frac{d \theta}{d x}=\frac{d \mu}{d N} \frac{1}{k_{F}(x)}$ with $\frac{d \mu}{d N}=N=\sqrt{2 \mu}$, and $\theta_{x^{-}}=0, \theta_{x^{+}}=\pi$. Using (S20) and trigonometric relations, one obtains explicit formula (not displayed here) for $\operatorname{Var} \mathcal{N}_{[a, b]}$ from (2) in the text for $a, b$ in the bulk, and for $\operatorname{Var} \mathcal{N}_{[0, a]}=\operatorname{Var} \mathcal{N}_{[a, L]}$ from (20) in the text, in the regime $\mathrm{a} \sim \mathrm{b} \sim \mu$. One can check that in the limit $\mathrm{a} \sim \mathrm{b} \ll \mu$ they agree with the Fisher-Hartwig asymptotics obtained using Riemann-Hilbert methods for the JUE in [89] (see also Section X A). Here we only display the result for the hard box $V(x)=0$ for $x \in[0, L]$ and Dirichlet boundary conditions for the wavefunction at $x=0, L$. It can be obtained as the limit of the JUE for $\mathrm{a}=\mathrm{b}=1 / 2$, i.e., $A=0$ and $B=1$. One has $\mu=\frac{\pi^{2}}{2 L^{2}} N^{2}, \frac{d \mu}{d N}=\frac{\pi^{2}}{L^{2}} N=\frac{\pi}{L} \sqrt{2 \mu}$ and $\theta_{x}=\pi \frac{x}{L}$ and from (20) and (2) in the text we obtain for the hard box (where $N=\frac{L}{\pi} \sqrt{2 \mu}$ )

$$
\begin{aligned}
& \operatorname{Var} \mathcal{N}_{[0, a]}=\operatorname{Var} \mathcal{N}_{[a, L]}=\frac{1}{2 \pi^{2}}\left(\log N+\log \left|\sin \pi \frac{a}{L}\right|+c_{2}+\log 2+o(1)\right), \\
& \operatorname{Var} \mathcal{N}_{[a, b]}=\operatorname{Var} \mathcal{N}_{[0, a]}+\operatorname{Var} \mathcal{N}_{[0, b]}+\frac{1}{\pi^{2}} \log \left|\frac{\sin \frac{\pi(a-b)}{2 L}}{\sin \frac{\pi(a+b)}{2 L}}\right|+o(1) .
\end{aligned}
$$

The result (S26) is compared with a numerical calculation in Fig. 5 in Section XI.

(ii) Non confining potential: single turning point. Consider here a general potential $V(x)$, such that the support of the bulk density is the semi-infinite interval $\left[x^{-},+\infty\left[, x^{-} \in \mathbb{R}\right.\right.$. One example for fermions in $[0,+\infty[$ is the repulsive inverse square wall, $V(x)=\frac{\alpha(\alpha-1)}{2 x^{2}}$, with $\alpha>1$, with $x^{-}=\sqrt{\frac{\alpha(\alpha-1)}{2 \mu}}$. Although the variable $\theta_{x}$ cannot be defined as in (3) in the text, one can still obtain the variance by taking the limit $x^{+} \rightarrow+\infty$. Consider the formula (20) and (2) in the text. In that limit $\int_{x^{-}}^{x^{+}} \frac{d x^{\prime}}{k_{F}\left(x^{\prime}\right)} \rightarrow+\infty$ but $\int_{x^{-}}^{x} \frac{d x^{\prime}}{k_{F}\left(x^{\prime}\right)}$ remains fixed. Hence $\theta_{x}$ becomes small and one can Taylor expand in it. We see that $\frac{\sin \theta_{a}}{d \mu / d N} \rightarrow \int_{x^{-}}^{a} \frac{d x^{\prime}}{k_{F}\left(x^{\prime}\right)}$ in (20) in the text leading to (for any $c^{-}<x^{-}$)

$$
(2 \pi)^{2} \operatorname{Var} \mathcal{N}_{\left[c^{-}, a\right]} \simeq(2 \pi)^{2} \operatorname{Var} \mathcal{N}_{[a,+\infty]} \simeq \log \left(2 k_{F}(a)^{2} \int_{x^{-}}^{a} \frac{d x^{\prime}}{k_{F}\left(x^{\prime}\right)}\right)+c_{2}=\log \mu+\log 4 \tilde{a}\left(1-\frac{\lambda^{2}}{4 \tilde{a}^{2}}\right)^{3 / 2}+c_{2}
$$

where the last equality is specialized to the inverse square wall, with $\lambda=\alpha / \mu$ and $\tilde{a}=a / \sqrt{2 \mu}$, in which case $\int_{x^{-}}^{a} \frac{d x^{\prime}}{k_{F}\left(x^{\prime}\right)}=\frac{a}{2 \mu} k_{F}(a)$, and it is valid in the limit $\alpha \rightarrow \infty, \mu \rightarrow \infty$ keeping $\lambda$ and $\tilde{a}$ fixed. Similarly, performing the limit $x^{+} \rightarrow+\infty$ on (2) in the text, one obtains the variance for an interval in the bulk, for a general such potential

$$
\pi^{2} \operatorname{Var} \mathcal{N}_{[a, b]}=\log \left(2 k_{F}(a) k_{F}(b) \int_{x^{-}}^{a} \frac{d z}{k_{F}(z)} \int_{x^{-}}^{b} \frac{d z}{k_{F}(z)}\right)+\log \left|\frac{\int_{a}^{b} \frac{d z}{k_{F}(z)}}{\int_{x^{-}}^{a} \frac{d z}{k_{F}(z)}+\int_{x^{-}}^{b} \frac{d z}{k_{F}(z)}}\right|+c_{2}+o(1) .
$$

For the inverse square wall, it is known that the kernel is the Bessel kernel $K_{\mu}(x, y)=2 k_{F}^{2} \sqrt{x y} K_{B e, \alpha-1 / 2}\left(k_{F}^{2} x^{2}, k_{F}^{2} y^{2}\right)$ with $k_{F}=k_{F}(+\infty)=\sqrt{2 \mu}$, and the set of $k_{F}^{2} x_{i}^{2}$ form a determinantal Bessel process of index $\alpha-1 / 2$. In [82] results are obtained for the Bessel process for fixed $\alpha$. The correspondence amounts to set in [82] $x=1$ and $r=k_{F}^{2} a^{2}=2 \mu a^{2}$, and $N_{[0, r]}$ there equal to our $N_{[0, a]}$. The formula (1.16) in [82] is obtained for $r \rightarrow+\infty$ at fixed $\alpha=O(1)$ and we see that it agrees with our result (S28) for $\lambda=0$. Note however that, while this paper was in progress, a new result was obtained [83] in the limit $\alpha \sim \mu$. This new result, obtained by very different methods, also agrees with our formula (S28) for generic $\lambda$. Similarly our formula (S28) for the interval $[a, b]$ can be compared with (1.19) in [82].

(iii) Fermions on the circle: no turning point. Consider fermions on the circle $x \in[0, L]$, and a periodic potential of period $L$ such that $V(x)<\mu$ for all $x$, i.e., without turning points. Let us first display our result, and then sketch how it is obtained from the results in Section IV (since it is slightly different from the other cases). We find that for any macroscopic interval $[a, b]$ the formula for the variance of $\mathcal{N}_{[a, b]}$ in Eqs. (2), (3) in the text are replaced, in the case of the circle, by

$$
\pi^{2} \operatorname{Var} \mathcal{N}_{[a, b]}=\log \left|\sin \frac{\theta_{a}-\theta_{b}}{2}\right|+\log \left(k_{F}(a) k_{F}(b) \int_{0}^{L} \frac{d z}{\pi k_{F}(z)}\right)+c_{2}+o(1), \theta_{x}:=2 \pi \frac{\int_{0}^{x} \frac{d z}{k_{F}(z)}}{\int_{0}^{L} \frac{d z}{k_{F}(z)}}, k_{F}(x)=\sqrt{2(\mu-V(x))} .
$$


Note the factor of 2 in the definition of $\theta_{x}$. In Section IV we computed $K_{\mu}(x, y)^{2}$ for the circle, given by (S18). To obtain (S30) we use that $\operatorname{Var} \mathcal{N}_{[a, b]}$, considered as a symmetric function of $(a, b)$, obeys (from Section III) $\frac{1}{2} \partial_{a} \partial_{b} \operatorname{Var} \mathcal{N}_{[a, b]}=$ $K_{\mu}(a, b)^{2}-\delta(a-b) \rho(a)$. The Eq. (S18) then determines $\operatorname{Var} \mathcal{N}_{[a, b]}$ up to a term $f(a)+f(b)$, and the function $f$ is then fixed using the matching onto the microscopic result, leading to (S30). Note that if one knows $\operatorname{Var} \mathcal{N}_{D}$ for any interval $\mathcal{D}=[a, b]$, one knows it for $\mathcal{D}$ being any collection of intervals, since e.g. one can always define a height function $h(a)=\mathcal{N}_{[0, a]}$ and use (S3),(S4).

Let us discuss some properties of the result (S30). For a microscopic interval $|a-b|=O\left(1 / k_{F}(a)\right)$ one has as usual, $\pi^{2} \operatorname{Var} \mathcal{N}_{[a, b]} \simeq U(0)-U\left(k_{F}(a)|a-b|\right)=\log \left[k_{F}(a)|a-b|\right]+c_{2}+o(1)$ with the same function $U(z)$ as in the text. One can check that (S30) matches with this microscopic result in two limits (i) $|a-b| \ll 1$ and (ii), due to the periodicity of the circle, $a \rightarrow 0$ and $b \rightarrow L$, using $\left|\sin \frac{\theta_{a}-\theta_{b}}{2}\right| \simeq \frac{\pi}{\int_{0}^{L} \frac{d z}{k_{F}(z)}}\left(\int_{0}^{a} \frac{d z}{k_{F}(z)}+\int_{b}^{L} \frac{d z}{k_{F}(z)}\right)$ which leads to $\operatorname{Var} N_{[a, b]} \simeq \log \left[k_{F}(0)(L-b+a)\right]+c_{2}+o(1)$ in that limit. Finally it is interesting to note that the formula to be used may change as $\mu$ is varied, e.g. for the potential $V(x)=V_{0} \cos 2 \pi x / L$ on the circle, for $\mu>V_{0}$ we must use (S30), while for $\mu<V_{0}$ we must use (2) in the text.

\section{CENTRAL POTENTIAL FOR $d>1$ : GENERALITIES AND DECOUPLING}

Here we consider noninteracting fermions in their ground state in a central potential $V(r)$. The $N$ body Hamiltonian is $\mathcal{H}_{N}=\sum_{i=1}^{N} \hat{H}_{i}$, where the single particle Hamiltonian $\hat{H}=\frac{\mathbf{p}^{2}}{2}+V(r)$. We show the decoupling between the different angular momentum sectors which leads to Eq. (23) in the text.

We use the spherical coordinates $\mathbf{x}=(r, \boldsymbol{\theta})$ where $\boldsymbol{\theta}$ is a $d-1$ dimensional angular vector. In these coordinates, the single particle Hamiltonian can be written as $\hat{H}=-\frac{1}{2} \Delta_{\mathbf{x}}+V(r)=-\frac{1}{2} r^{1-d} \partial_{r}\left(r^{d-1} \partial_{r}\right)+\frac{1}{2 r^{2}} \hat{\boldsymbol{L}}^{2}+V(r)$. The eigenfunctions of $\hat{H}$, using spherical symmetry, are labeled by the quantum numbers $(n, \mathbf{L})$, where $n$ is a positive integer, and where $\mathbf{L}$ stands collectively for all the angular quantum numbers. They can be written as [80]

$$
\psi_{n, \mathbf{L}}(r, \boldsymbol{\theta})=r^{\frac{1-d}{2}} \chi_{n, l}(r) Y_{\mathbf{L}}(\boldsymbol{\theta}) .
$$

The $Y_{\mathbf{L}}(\boldsymbol{\theta})$ are the $d$-dimensional spherical harmonics, labeled by the set of angular quantum numbers $\mathbf{L}$. They are eigenfunctions of $\hat{\boldsymbol{L}}^{2}$ with eigenvalues $\ell(\ell+d-2)$ depending on a single nonnegative integer $\ell$. The radial part $\chi_{n, \ell}(r)$ is the eigenfunction of a $1 d$ effective Hamiltonian, $\hat{H}_{\ell} \chi_{n, \ell}=\epsilon_{n, \ell} \chi_{n, \ell}$, with an effective potential

$$
V_{\ell}(r)=V(r)+\frac{\left(\ell+\frac{d-3}{2}\right)\left(\ell+\frac{d-1}{2}\right)}{2 r^{2}} .
$$

The ground state wavefunction is given by the Slater determinant $\Psi_{0}\left(\mathbf{x}_{\mathbf{1}}, \cdots, \mathbf{x}_{\mathbf{N}}\right)=\frac{1}{\sqrt{N !}} \operatorname{det}_{1 \leq i, j \leq N}\left[\psi_{\mathbf{k}_{i}}\left(\mathbf{x}_{j}\right)\right]$, where $\mathbf{k}_{i}=\left(n_{i}, \mathbf{L}_{i}\right)$ labels the single particle eigenfunction of the occupied eigenstates. In the ground-state, the occupied eigenstates are all the energy levels $\epsilon_{n, \ell} \leq \mu$, where $\mu$ is the Fermi energy (we assume for simplicity that $N$ is such that the many-body ground state is not degenerate). Using standard methods, such as the Cauchy-Binet formula (see e.g. $[43,80]$ ) we can write the generating function of the cumulants of the number of fermions $\mathcal{N}_{R}$ in a sphere of radius $R$ centered at the origin using the overlap matrix $\mathbb{A}$

$$
\left\langle e^{-s \mathcal{N}_{R}}\right\rangle=\operatorname{det}_{1 \leq i, j \leq N}\left[\delta_{i j}-\left(1-e^{-s}\right) \mathbb{A}_{i j}\right] \quad, \quad \mathbb{A}_{i j}=\int_{r=|\mathbf{x}| \leq R} d^{d} \mathbf{x} \psi_{n_{i}, \mathbf{L}_{i}}^{*}(\mathbf{x}) \psi_{n_{j}, \mathbf{L}_{j}}(\mathbf{x}),
$$

where $\psi_{n, \mathbf{L}}(\mathbf{x})$ is given in Eq. (S31). and $\langle\ldots\rangle$ denotes the quantum expectation value with respect to $\left|\Psi_{0}\right|^{2}$. Using $d^{d} \mathbf{x}=r^{d-1} d r d \boldsymbol{\theta}$ and the orthogonality property of the spherical harmonics $\int d \boldsymbol{\theta} Y_{\mathbf{L}}(\boldsymbol{\theta}) Y_{\mathbf{L}^{\prime}}(\boldsymbol{\theta})=\delta_{\mathbf{L}, \mathbf{L}^{\prime}}$ the angular integral gives

$$
\int_{r \leq R} d \mathbf{x} \psi_{n_{i}, \mathbf{L}_{i}}^{*}(\mathbf{x}) \psi_{n_{j}, \mathbf{L}_{j}}(\mathbf{x})=\delta_{\mathbf{L}_{i}, \mathbf{L}_{j}} \int_{0}^{R} d r \chi_{n_{i}, \ell_{i}}(r) \chi_{n_{j}, \ell_{i}}(r)
$$

where we recall that $\int_{0}^{\infty} d r \chi_{k l}(r) \chi_{k^{\prime} l}(r)=\delta_{k k^{\prime}}$. Hence the overlap matrix $\mathbb{A}$ is diagonal in the variables $\mathbf{L}_{i}$ and the determinant factorises over the different angular sectors. Therefore Eq. (S33) takes the product form

$$
\left\langle e^{-s \mathcal{N}_{R}}\right\rangle=\prod_{\ell \geq 0}^{\ell_{\max }(\mu)} Z_{\ell}\left(s, m_{\ell}\right)^{g_{d}(\ell)} \quad, \quad Z_{\ell}\left(s, m_{\ell}\right)=\left\langle e^{\left.-s \mathcal{N}_{[0, R]}\right\rangle_{\ell}}=\operatorname{det}_{1 \leq i, j \leq m_{\ell}}\left[\delta_{i j}-\left(1-e^{-s}\right) \int_{0}^{R} d r \chi_{i, \ell}(r) \chi_{j, \ell}(r)\right]\right.
$$


where $g_{d}(\ell)=\frac{(2 \ell+d-2) \Gamma(\ell+d-2)}{\Gamma(\ell+1) \Gamma(d-1)}$ are the (angular) degeneracies with $g_{d}(0)=1$ for all $d \geq 1$ and $g_{1}(0)=g_{1}(1)=1$ together with $g_{1}(\ell)=0$ for all $\ell \geq 2$. Here $Z_{\ell}\left(s, m_{\ell}\right)$ is the generating function of cumulants of the number of fermions $\mathcal{N}_{[0, R]}$ in the interval $r \in[0, R]$ for the $1 \mathrm{~d}$ system of $m_{\ell}$ fermions described by the single particle Hamiltonian $\hat{H}_{\ell}$. Taking the logarithm in (S35) and expanding in $s$ one obtains the equation (23) in the text. Note that the above arguments extends to any domain with radial symmetry, for instance the spherical shell $R_{1}<r<R_{2}$ which maps onto the study of the interval $\left[R_{1}, R_{2}\right]$ in one dimension. Note that the proof given here of $(23)$ in the text assumes that the total number of fermions $N$ is finite (and the same for the $m_{\ell}$ ), which is natural for a confining potential. However it also applies to the case where the potential is non confining, e.g. for free fermions $V(r)=0$, as can be seen by taking a limit where the right edge tends to infinity with fixed $R$.

This property is even more general, as can be understood by the following physical argument. The single-particle angular momentum $\hat{\boldsymbol{L}}$ and the radial distance operator $\hat{r}$, commute and can therefore be measured simultaneously. The measurement of $\hat{\boldsymbol{L}}^{2}$ in the ground state leads to the values $\left\{\ell_{i}\right\}_{i=1,2, \ldots, \mathcal{M}}$ of all of the angular sectors which have a nonzero number of particles, where each value of $\ell$ appears in this list $g_{d}(\ell)$ times and $\mathcal{M}=\sum_{\ell=0}^{\ell_{\max }(\mu)} g_{d}(\ell)$. Since after the measurement, the Pauli exclusion principle only acts between the particles in the same angular sector we find that the radial JPDF decouples. Although the way to write it, which we show here for illustration, is a bit heavy because one must ensure the global symmetry of the JPDF, the concept of this decoupling is quite simple. Defining $a_{i}=\sum_{j<i} m_{\ell_{j}}$, and recalling that $N=\sum_{i=1}^{\mathcal{M}} m_{\ell_{i}}$, we can write

$$
P\left(r_{1}, \ldots, r_{N}\right)=\frac{1}{N !} \sum_{\tau \in S_{N}} \prod_{i=1}^{\mathcal{M}} P_{\ell_{i}}\left[r_{\tau\left(a_{i}+1\right)}, \ldots, r_{\tau\left(a_{i}+m_{l_{i}}\right)}\right]=\frac{\prod_{i=1}^{\mathcal{M}}\left(m_{\ell_{i}}\right) !}{N !} \sum_{\substack{\cup_{i=1}^{\mathcal{M}} A_{\ell_{i}=\{1, \ldots, N\}} \\ \forall i \neq j, A_{\ell_{i}} \cap A_{\ell_{j}}=\emptyset, \forall i,\left|A_{\ell_{i}}\right|=m_{\ell_{i}}}} \prod_{i=1}^{\mathcal{M}} P_{\ell_{i}}\left(\vec{r}_{A_{\ell_{i}}}\right)
$$

where $P_{\ell}\left(x_{1}, \ldots, x_{m_{\ell}}\right)$ is the joint PDF of the positions of $m_{\ell}$ noninteracting fermions in the 1d potential (S32). (and $S_{N}$ is the group of permutations of the set $\{1, \ldots, N\}$ ). The last formula involves a sum over the partitions of the set $\{1, \ldots, N\}$ into $\mathcal{M}$ subsets $\left\{A_{\ell_{i}}\right\}_{i=1, \ldots, \mathcal{M}}$. The last equality arises by regrouping the terms in the first formula according to the values of the sets $A_{\ell_{i}}=\left\{\tau\left(a_{i}+1\right), \ldots, \tau\left(a_{i}+m_{\ell_{i}}\right)\right\}$ and using the symmetry of each $P_{\ell_{i}}\left(\left\{r_{j}\right\}_{j \in A_{\ell_{i}}}\right) \equiv P_{\ell_{i}}\left(\vec{r}_{A_{\ell_{i}}}\right)$ when summing over permutations. Finally, Eq. (23) in the text now follows from the fact that cumulants of sums of independent random variables are the sum of their cumulants.

\section{FREE FERMIONS IN DIMENSION $d$}

We give here some details about the derivation of the variance for free fermions, i.e., for $V(r)=0$ in dimension $d$ given in the text, together with an alternative method, and compare with known results.

\section{A. Free fermions, using the decoupling and the 1D inverse square potential}

Using the results of section VI, the case of free fermions $\hat{H}=\frac{\mathbf{p}^{2}}{2}$ in dimension $d$ can be studied using the $1 \mathrm{~d}$ Hamiltonian $\hat{H}_{\ell}=-\frac{1}{2} \frac{\partial^{2}}{\partial r^{2}}+V_{\ell}(r)$ with $V_{\ell}(r)=\frac{\alpha(\alpha-1)}{2 r^{2}}$ with $\alpha=\ell+\frac{d-1}{2}$. Consider the number $\mathcal{N}_{R}$ of fermions in the sphere of radius $R$. From (23) in the text, its average is given by

$$
\left\langle\mathcal{N}_{R}\right\rangle=\sum_{\ell=0}^{+\infty} g_{d}(\ell)\left\langle\mathcal{N}_{[0, R]}\right\rangle_{\ell} .
$$

Note that since the potential is not confining the sum over $\ell$ extends to infinity. However, within the sector of angular momentum $\ell$, the support of the density at large $\mu$ is $\left[r^{-}(\ell),+\infty\left[\right.\right.$ with $r^{-}(\ell) \simeq \ell / \sqrt{2 \mu}$ for $\ell \gg 1$. Hence, for a fixed $R$, the sum is effectively cutoff at $\ell=\ell_{c}(\mu, R)=k_{F} R$ with $k_{F}=\sqrt{2 \mu}$. Using the $1 \mathrm{~d}$ bulk density we obtain, by replacing the sum in (S37) by an integral (which is justified for large $\mu$ )

$$
\left\langle\mathcal{N}_{R}\right\rangle \simeq \int_{0}^{\sqrt{2 \mu} R} d \ell \frac{2 \ell^{d-2}}{\Gamma(d-1)} \int_{0}^{R} d r \frac{1}{\pi} \sqrt{\left(2 \mu-\frac{\ell^{2}}{r^{2}}\right)_{+}}=S_{d} \int_{0}^{R} d r r^{d-1} \frac{\mu^{d / 2}}{(2 \pi)^{d / 2} \Gamma(1+d / 2)}=\frac{\left(k_{F} R\right)^{d}}{2^{d} \Gamma(1+d / 2)^{2}}
$$

where $(x)_{+}=\max (x, 0), S_{d}=2 \pi^{d / 2} / \Gamma(d / 2)$ is the area of the unit sphere embedded in dimension $d$, and the sum is dominated by values of $\ell \gg 1$, with $g_{d}(\ell) \simeq \frac{2 \ell^{d-2}}{\Gamma(d-1)}$ for $d>1$. This agrees with the standard result for free fermions for 
any $d \geq 1$. Furthermore, the first two equalities in (S38) also hold for an arbitrary potential $V(r)$ upon substituting $\mu \rightarrow \mu-V(r)$ (and $\mu^{d / 2} \rightarrow(\mu-V(r))_{+}^{d / 2}$ ), recovering the known result for the density in the bulk Eq. (180) in [11]. This is a good test of the method.

Consider now the variance of $\mathcal{N}_{R}$. Using (23) in the text we can proceed similarly as in (S38) and use the asymptotic result for the variance of $\mathcal{N}_{[0, R]}$ in (S28), with the substitution $\tilde{a} \rightarrow R / \sqrt{2 \mu}$ and $\lambda=\frac{\alpha(\alpha-1)}{\mu^{2}} \simeq \ell^{2} / \mu^{2}$ since the sum is again dominated by large values of $\ell$

$$
\left\langle\mathcal{N}_{R}^{2}\right\rangle^{c}=\sum_{\ell=0}^{\ell_{\max }(\mu)} g_{d}(\ell)\left\langle\mathcal{N}_{[0, R]}^{2}\right\rangle_{\ell}^{c} \simeq \int_{0}^{\sqrt{2 \mu} R} d \ell \frac{2 \ell^{d-2}}{\Gamma(d-1)} \frac{1}{2 \pi^{2}}\left(\log \mu+\log 4 \tilde{a}\left(1-\frac{\lambda^{2}}{4 \tilde{a}^{2}}\right)^{3 / 2}+c_{2}\right)
$$

Performing the change of variable $\ell=\sqrt{2 \mu} R \Lambda$ and integrating over $\Lambda$, using $(1-d) \int_{0}^{1} d \Lambda \Lambda^{d-2} \log \left(1-\Lambda^{2}\right)=$ $\psi^{(0)}\left(\frac{d+1}{2}\right)+\gamma_{E}, \psi^{(0)}(x)$ being the di-gamma function, one finds

$$
\operatorname{Var} \mathcal{N}_{R} \simeq \frac{\left(k_{F} R\right)^{d-1}}{\pi^{2} \Gamma(d)}\left[\log \left(k_{F} R\right)+1-\frac{1}{2} \gamma_{E}+2 \log 2-\frac{3}{2} \psi^{(0)}\left(\frac{d+1}{2}\right)\right] \quad, \quad k_{F}=\sqrt{2 \mu} .
$$

This formula gives the first two orders in an expansion in the dimensionless parameter $k_{F} R \gg 1$ for any $d \geq 1$ [116].

Remark One can similarly calculate the variance of the number of fermions in a spherical shell $R_{1}<r<R_{2}$ using (S29) upon substituting $a \rightarrow R_{1}, b \rightarrow R_{2}$ and $\alpha(\alpha-1) \rightarrow \ell^{2}$ and using $\int_{x^{-}}^{a} \frac{d x^{\prime}}{k_{F}\left(x^{\prime}\right)}=\frac{a}{2 \mu} k_{F}(a)$ and $k_{F}(x)=\sqrt{2 \mu-\frac{\ell^{2}}{x^{2}}}$.

Comparison with known results. The leading term is already known from various works, with quite different methods, as we now discuss. However the subleading term in (S40) is to our knowledge new. The term $\sim R^{d-1} \log R$ was explicitly computed for a $d$ dimensional sphere in Ref. [42], Eq. (56) (in units such that the density is unity).

The variance of $\mathcal{N}_{\mathcal{D}}$ was given to leading order for an arbitrary domain $\mathcal{D}$ in [41, 43], based on a conjecture of Widom [38-40], for free fermions described by the kernel $K_{\mu}(\mathbf{x}, \mathbf{y})=\int_{\Gamma(\mu)} \frac{d^{d} \mathbf{k}}{(2 \pi)^{d}} e^{i \mathbf{k} \cdot(\mathbf{x}-\mathbf{y})}$ where $\Gamma(\mu)$ is the Fermi volume. Let $\Omega$ be a fixed domain in $\mathbb{R}^{d}$ and $\mathcal{D}$ the domain obtained from $\Omega$ by rescaling space by $R$, then for large $R$

$$
\operatorname{Var} \mathcal{N}_{\mathcal{D}} \simeq \frac{1}{(2 \pi)^{d-1}} \frac{1}{4 \pi^{2}} R^{d-1} \log R \int_{\partial \Omega} d \mathbf{S}_{x} \int_{\partial \Gamma(\mu)} d \mathbf{S}_{k}\left|\mathbf{n}_{k} \cdot \mathbf{n}_{x}\right|
$$

where $\partial \Omega$ and $\partial \Gamma(\mu)$ are the boundaries of $\Omega$ and of the Fermi volume, and $n_{x}$ and $n_{k}$ the respective unit vectors. In our present case upon rescaling we can reduce to an integral over two unit spheres, which can be written as an integral over a single unit sphere

$$
\operatorname{Var} \mathcal{N}_{\mathcal{D}} \simeq \frac{1}{(2 \pi)^{d-1}} \frac{1}{4 \pi^{2}}\left(k_{F} R\right)^{d-1} \log \left(k_{F} R\right) S_{d}^{2} \frac{\int_{0}^{\pi} d \theta(\sin \theta)^{d-2}|\cos \theta|}{\int_{0}^{\pi} d \theta(\sin \theta)^{d-2}} \simeq \frac{1}{\pi^{2} \Gamma(d)}\left(k_{F} R\right)^{d-1} \log \left(k_{F} R\right)
$$

where $k_{F}^{d-1}$ arises from rescaling of the integral over $\partial \Gamma(\mu)$ and we added a subleading term $\propto\left(k_{F} R\right)^{d-1} \log k_{F}$. We see that (S42) agrees with the leading term of our result (S40). Note also the recent work [45] where a higher dimensional bosonisation method was used to recover the leading order term of $\operatorname{Var} \mathcal{N}_{R}$ for free fermions in $d=2$. At this stage however this method does not predict the subleading term analytically. The authors of [45] provided a numerical determination of this term which, as we checked, is in excellent agreement with our analytical result Eq. (S40)

\section{B. Derivation of the free fermion result from the $d$-dimensional kernel}

Here we provide a direct calculation of the variance of $\mathcal{N}_{R}$ for free fermions (i.e., $\left.V(r)=0\right)$ in the infinite space in any dimension $d$. The exact kernel in that case, i.e., the $d$ dimensional analog of the sine kernel is given by [11, 42, 88]

$$
K_{\mu}(\mathbf{x}, \mathbf{y})=\int_{k<k_{F}} \frac{d^{d} \mathbf{k}}{(2 \pi)^{d}} e^{i \mathbf{k} \cdot(\mathbf{x}-\mathbf{y})}=\left(\frac{k_{F}}{2 \pi x}\right)^{d / 2} J_{d / 2}\left(k_{F}|\mathbf{x}-\mathbf{y}|\right) .
$$

where $x=|\mathbf{x}|$ and $k_{F}=\sqrt{2 \mu}$ is related to the uniform density via $\rho(\mathbf{x})=K_{\mu}(\mathbf{x}, \mathbf{x})=\frac{k_{F}^{d}}{2^{d} \pi^{d / 2} \Gamma(1+d / 2)}$. The variance of $\mathcal{N}_{R}$ is given by

$$
\operatorname{Var} \mathcal{N}_{R}=W_{1}-W_{2} \quad, \quad W_{1}=\int_{x<R} d^{d} \mathbf{x} \rho(\mathbf{x}) \quad, \quad W_{2}=\int_{x<R} d^{d} \mathbf{x} \int_{y<R} d^{d} \mathbf{y} K_{\mu}(\mathbf{x}, \mathbf{y})^{2} .
$$


One obtains $W_{1}=\left(k_{F} R\right)^{d} \frac{S_{d}}{2^{d} \pi^{d / 2} d \Gamma(1+d / 2)}=\left(k_{F} R\right)^{d} \frac{1}{2^{d} \Gamma^{2}(1+d / 2)}$ where we used the uniform density given above and the area of the unit sphere embedded in $d$ dimensions, $S_{d}=2 \pi^{d / 2} / \Gamma(d / 2)$. The second term, $W_{2}$, in (S44) can be written as

$$
W_{2}=\int_{k<k_{F}} \frac{d^{d} \mathbf{k}}{(2 \pi)^{d}} \int_{k^{\prime}<k_{F}} \frac{d^{d} \mathbf{k}^{\prime}}{(2 \pi)^{d}} A\left(\mathbf{k}+\mathbf{k}^{\prime}\right) \quad, \quad A(\mathbf{p}):=\int_{x<R} d^{d} \mathbf{x} \int_{y<R} d^{d} \mathbf{y} e^{i \mathbf{p} \cdot(\mathbf{x}-\mathbf{y})}=\left(\frac{2 \pi R}{p}\right)^{d} J_{d / 2}^{2}(p R) .
$$

One can rewrite $W_{2}$ as

$$
W_{2}=\int d^{d} \mathbf{p} A(\mathbf{p}) B(\mathbf{p}) \quad, \quad B(\mathbf{p}):=\int_{k<k_{F}} \frac{d^{d} \mathbf{k}}{(2 \pi)^{d}} \int_{k^{\prime}<k_{F}} \frac{d^{d} \mathbf{k}^{\prime}}{(2 \pi)^{d}} \delta^{d}\left(\mathbf{p}-\left(\mathbf{k}+\mathbf{k}^{\prime}\right)\right)=\int \frac{d^{d} \mathbf{z}}{(2 \pi)^{d}} e^{i \mathbf{z} \cdot \mathbf{p}}\left(\frac{k_{F}}{2 \pi z}\right)^{d} J_{d / 2}^{2}\left(k_{F} z\right)
$$

where we used the integral representation of the delta function over the $\mathbf{z}$ variable. In Eq. (S46) the integrals over $\mathbf{p}$ and $\mathbf{z}$ run over $\mathbb{R}^{d}$. Hence rescaling $\mathbf{z} \rightarrow \mathbf{z} / k_{F}$ and $\mathbf{p} \rightarrow \mathbf{p} / R$ we obtain the following scaling form for the variance

$$
\operatorname{Var} \mathcal{N}_{R}=\mathcal{U}_{d}\left(k_{F} R\right), \mathcal{U}_{d}(x)=x^{d}\left(\frac{1}{2^{d} \Gamma^{2}(1+d / 2)}-\frac{1}{(2 \pi)^{d}} \int d^{d} \mathbf{z} \int d^{d} \mathbf{p} e^{i \frac{\mathbf{z} \cdot \mathbf{p}}{x}} \frac{1}{z^{d} p^{d}} J_{d / 2}^{2}(p) J_{d / 2}^{2}(z)\right)
$$

where the scaling function generalizes the one obtained in $d=1$ below Eq. (17) in the main text, in the following sense $\lim _{d \rightarrow 1} \mathcal{U}_{d}(x)=\frac{1}{\pi^{2}}(U(0)-U(2 x))$, where $U(0)=c_{2}$.

The case $d=2$ : in this case the double integral (S47) reads

$$
\begin{aligned}
w_{2} & =\frac{1}{(2 \pi)^{2}} \int \frac{d^{2} z}{|z|^{2}} J_{1}^{2}(|z|) \int_{0}^{\infty} \frac{p d p}{p^{2}} \int_{0}^{2 \pi} d \theta e^{i \frac{z p}{x} \cos \theta} J_{1}^{2}(p)=\left(k_{F} R\right)^{2} \int_{0}^{\infty} \frac{d z}{z} J_{1}^{2}(z) \int_{0}^{\infty} \frac{d p}{p} J_{0}\left(\frac{z p}{k_{F} R}\right) J_{1}^{2}(p) \\
& =\frac{1}{\pi} \int_{0}^{2 x} \frac{d z}{z} J_{1}^{2}(z)\left[\cos ^{-1}\left(\frac{z}{2 x}\right)-\frac{z}{2 x} \sqrt{1-\left(\frac{z}{2 x}\right)^{2}}\right] .
\end{aligned}
$$

This leads to

$$
\mathcal{U}_{2}(x)=\frac{x^{2}}{4}\left[{ }_{2} F_{3}\left(\left\{\frac{1}{2}, \frac{1}{2}\right\},\{1,1,2\},-4 x^{2}\right)+\frac{x^{2}}{4}{ }_{2} F_{3}\left(\left\{\frac{3}{2}, \frac{3}{2}\right\},\{2,3,3\},-4 x^{2}\right)\right] .
$$

This function is plotted in Fig. 6 (b) in Section XI. For large $x$ it behaves as $\mathcal{U}_{2}(x)=\frac{x}{\pi^{2}}\left(\ln x+\gamma_{E}-2+5 \ln 2+o(1)\right)$, which agrees with Eq. (S40) for $d=2$ using that $\psi^{(0)}(3 / 2)=2-2 \ln 2-\gamma_{E}$. Note that the subleading terms are actually of order $O(1 / \sqrt{x})$ and rapidly oscillating.

The case $d>2$. In general $d$ the computation is more complicated, and leads to the following expression

$$
\mathcal{U}_{d}(x)=x^{d}\left(\frac{1}{2^{d} \Gamma^{2}(1+d / 2)}-\frac{2^{1-d}}{\Gamma(d / 2)} \int_{0}^{1} \frac{d u}{u} J_{d / 2}^{2}(2 x u)\left[\frac{1}{\Gamma(1+d / 2)}-\frac{2 u}{\sqrt{\pi} \Gamma((1+d) / 2)}{ }_{2} F_{1}\left(\frac{1}{2}, \frac{1-d}{2} ; \frac{3}{2} ; u^{2}\right)\right]\right) .
$$

In $d=3$ this integral can be performed explicitly

$$
\begin{aligned}
\mathcal{U}_{3}(x)=\frac{1}{288 \pi^{2}}\{ & \left(12-144 x^{2}\right) \operatorname{Ci}(4 x)-128 x^{3} \operatorname{Si}(4 x)+8(8 \pi x-9) x^{2}+12 \gamma_{E}\left(12 x^{2}-1\right) \\
& \left.-\left(32 x^{2}+5\right) \cos (4 x)-12 \log (x)+4 x[36 x \log (4 x)+7 \sin (4 x)]+5-24 \log (2)\right\},
\end{aligned}
$$

where $\operatorname{Si}(z)=\int_{0}^{z}[\sin (t) / t] d t$ and $\operatorname{Ci}(z)=-\int_{z}^{\infty}[\cos (t) / t] d t$ are the sine integral and cosine integral respectively. For $x \gg 1$,

$$
\mathcal{U}_{3}(x)=\frac{x^{2}}{2 \pi^{2}}\left(\ln x+\gamma_{E}+2 \ln 2-\frac{1}{2}\right)-\frac{1}{24 \pi^{2}} \ln x+\frac{1}{288 \pi^{2}}\left(5-12 \gamma_{E}-24 \ln 2\right)+o(1)
$$

$\mathcal{U}_{3}(x)$ is plotted in Fig. 6 (c) in Section XI. Using $\psi^{(0)}(2)=1-\gamma_{E}$, one can check that the leading order in (S52) indeed coincides with (S40) for $d=3$. We have checked the agreement for $d>3$.

For arbitrary dimension, at $k_{F} R \ll 1, \mathcal{N}_{\mathcal{R}}$ becomes a Bernoulli random variable, so $\operatorname{Var} \mathcal{N}_{R} \simeq\left\langle\mathcal{N}_{R}\right\rangle-\left\langle\mathcal{N}_{R}\right\rangle^{2}$. Indeed, one can check that the corresponding approximate equality $W_{2} \simeq W_{1}^{2}$ holds in this limit. This fact is also evident in the $x \ll 1$ behavior of the functions $\mathcal{U}_{d}(x)$. 


\section{GENERAL CENTRAL POTENTIAL IN DIMENSION $d$ AND HARMONIC OSCILLATOR}

We now consider the case of noninteracting fermions in a general central potential in $d$ dimension with a single particle Hamiltonian $\hat{H}=\frac{\mathbf{p}^{2}}{2}+V(r)$. Consider the number $\mathcal{N}_{R}$ of fermions in the sphere of radius $R$. Using the results of section VI, we study its statistics using the $1 \mathrm{~d}$ Hamiltonian $\hat{H}_{\ell}=-\frac{\partial^{2}}{\partial r^{2}}+V_{\ell}(r)$ with $V_{\ell}(r)=V(r)+\frac{\alpha(\alpha-1)}{2 r^{2}}$ with $\alpha=\ell+\frac{d-1}{2}$. We focus here on the large $\mu$ limit and we determine the cumulants of $\mathcal{N}_{R}$ using (23) in the text. In that limit the sum is dominated by values $\ell \gg 1$, hence we will approximate $V_{\ell}(r) \simeq V(r)+\frac{\ell^{2}}{2 r^{2}}$.

In dimension $d$ the bulk density is known to be given as $\rho^{\text {bulk }}(\mathbf{x})=\frac{1}{2^{d} \pi^{d / 2} \Gamma(1+d / 2)} k_{F}(r)^{d}$, where $k_{F}(r)=$ $\sqrt{2(\mu-V(r))_{+}}$. We first assume that (i) $V(r)$ is confining so that the bulk density is supported on the sphere of radius $r_{e}$, where $r_{e}$ is the unique root of $V\left(r_{e}\right)=\mu$. (ii) For $\ell>0, V_{\ell}(r)$ has either exactly two turning points, i.e., two roots $r^{ \pm}(\ell)$ to the equation $V_{\ell}\left(r^{ \pm}(\ell)\right)=\mu$, or none. The bulk density of the associated $1 \mathrm{~d}$ fermion problem, $\rho_{\ell}^{\text {bulk }}(r)=\sqrt{2\left(\mu-V_{\ell}(r)\right)_{+}} / \pi=\sqrt{\left(\left(r k_{F}(r)\right)^{2}-\ell^{2}\right)_{+}} /(\pi r)$ is thus non zero in the interval $r \in\left[r^{-}(\ell), r^{+}(\ell)\right]$ (where $V_{\ell}(r) \leq \mu$, equivalently $r k_{F}(r) \geq \ell$ ) and vanishes outside (where $V_{\ell}(r)>\mu$ ). These assumptions are equivalent to asking that the function $r \rightarrow r k_{F}(r)$ vanishes at $r=0$ and for $r \geq r_{e}$, and has a unique maximum at some $r=r^{*}$, see Figure 2. The equation $r k_{F}(r)=\ell$ has thus exactly two roots, $r^{ \pm}(\ell)$, for $r<r^{*}$, which annihilate at $\ell=\ell^{*}$. The harmonic oscillator $V(r) \sim r^{2}$ satisfies these assumptions. We will discuss later more general cases.

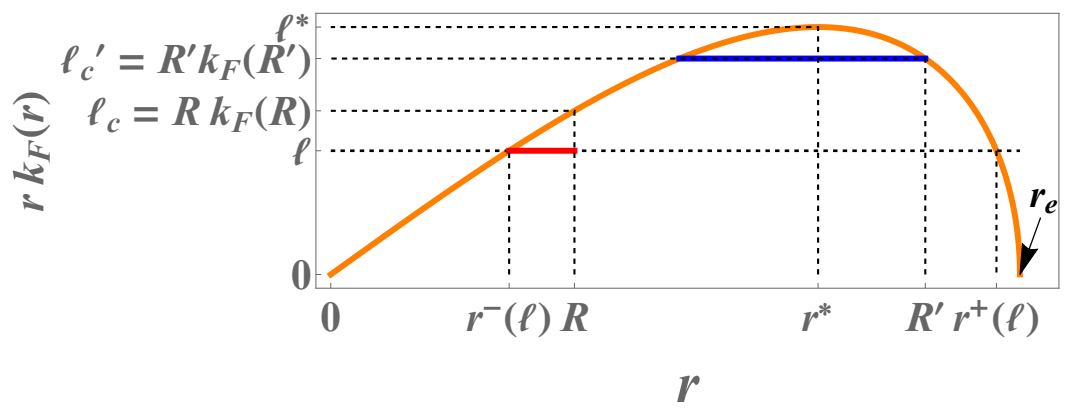

FIG. 2. Plot of the function $r \rightarrow r k_{F}(r)=r \sqrt{2 \mu-V(r)}$ (for the HO for illustration). In this case for each $0<\ell<\ell^{*}$ there are only two semi-classical turning points for the $1 \mathrm{~d}$ problem associated to $V_{\ell}(r)$, i.e., the two roots $r^{-}(\ell)<r^{+}(\ell)$ of $r k_{F}(r)=\ell$. The contribution $\left\langle\mathcal{N}_{[0, R]}^{2}\right\rangle_{\ell}^{c}$ of the sector of angular momentum $\ell$ to the variance of $\mathcal{N}_{R}$ in the sum in Eq. (23) in the text is given by the variance of $\mathcal{N}_{[0, R]}$ which includes a macroscopic number of particles, concentrated on the subinterval $\left[r^{-}(\ell), R\right]$ highlighted in red. For $\ell \geq \ell_{c}=\ell_{c}=R k_{F}(R)$ this subinterval becomes empty hence $\left\langle\mathcal{N}_{[0, R]}^{2}\right\rangle_{\ell}^{c} \simeq 0$. Similarly for $R^{\prime}>r^{*}$, when $\ell \geq \ell_{c}^{\prime}=R^{\prime} k_{F}\left(R^{\prime}\right)$ the interval $\left[r^{-}(\ell), R\right]$ shown in blue, becomes full (i.e., it contains $m_{\ell}$ fermions), hence the contribution $\left\langle\overline{\mathcal{N}}_{[0, R]}^{2}\right\rangle_{\ell}^{c}$ to the variance also vanishes for $\ell \geq \ell_{c}^{\prime}$.

Using (23) in the text and proceeding as in (S38), the average number of fermions in a sphere of radius $R$ is obtained as a sum over the bulk densities of the $1 \mathrm{~d}$ problems, i.e., $\left\langle\mathcal{N}_{R}\right\rangle \simeq \int_{0}^{+\infty} d \ell \frac{2 \ell^{d-2}}{\Gamma(d-1)} \int_{0}^{R} d r \rho_{\ell}^{\text {bulk }}(r)$, where the densities $\rho_{\ell}^{\text {bulk }}(r)$ vanish for $\ell>r k_{F}(r)$. From the remark below (S38), integration over $\ell$ recovers the result for $\rho^{\text {bulk }}(\mathbf{x})$ given above.

We now calculate the variance $\operatorname{Var} \mathcal{N}_{R}=\left\langle\mathcal{N}_{R}^{2}\right\rangle^{c}$ using formula (23) in the text for $p=2$. For the variance $\left\langle\mathcal{N}_{[0, R]}^{2}\right\rangle_{\ell}^{c}$ of the $1 \mathrm{~d}$ problem with potential $V_{\ell}$, we use the formula (20) of the text, since for that potential $\operatorname{Var} \mathcal{N}_{[0, R[}=H(R, R)$, and we recall that in this formula $\frac{d \mu}{d N} \simeq \frac{\pi}{\int_{x^{-}}^{x^{+}} d z / k_{F}(z)}$. One must substitute $a \rightarrow R, x \rightarrow r, x^{ \pm} \rightarrow r^{ \pm}(\ell), k_{F}(x) \rightarrow$ $\sqrt{2\left(\mu-V_{\ell}(r)\right)}=\frac{1}{r} \sqrt{\left(r k_{F}(r)\right)^{2}-\ell^{2}}$, leading to the general formula for the variance

$\operatorname{Var} \mathcal{N}_{R} \simeq$

$\frac{1}{2 \pi^{2}} \int_{0}^{R k_{F}(R)} \frac{2 \ell^{d-2} d \ell}{\Gamma(d-1)}\left[\log \left(\left(\left(R k_{F}(R)\right)^{2}-\ell^{2}\right) \int_{r^{-}(\ell)}^{r^{+}(\ell)} \frac{r d r}{\pi R^{2} \sqrt{r^{2} k_{F}(r)^{2}-\ell^{2}}}\right)+\log 2 \mid \sin \pi \frac{\int_{r^{-}(\ell) \frac{r d r}{\sqrt{r^{2} k_{F}(r)^{2}-\ell^{2}}}}^{\int_{r^{-}(\ell)}^{r^{+}(\ell)} \frac{r d r}{\sqrt{r^{2} k_{F}(r)^{2}-\ell^{2}}}} \mid+c_{2}}{]}\right.$

where we recall that $k_{F}(r)=\sqrt{2(\mu-V(r))}$. Note that within the $\ell$ sector, the number of fermions $\mathcal{N}_{[0, R]} \simeq 0$ for $R<r^{-}(\ell)$ and $\mathcal{N}_{[0, R]} \simeq m_{\ell}$ for $R>r^{+}(\ell)$, so the $1 \mathrm{~d}$ variance $\left\langle\mathcal{N}_{[0, R]}^{2}\right]_{\ell}^{c}$ is non zero only when $R \in\left[r_{-}(\ell), r_{+}(\ell)\right]$ with $\left\langle\mathcal{N}_{[0, R]}^{2}\right\rangle_{\ell}^{c} \simeq\left\langle\mathcal{N}_{\left[r^{-}(\ell), R\right]}^{2}\right\rangle_{\ell}^{c}=\left\langle\mathcal{N}_{\left[R, r^{+}(\ell)\right]}^{2}\right\rangle_{\ell}^{c}$. This explains the upper bound in (S53) since when $\ell$ reaches $\ell_{c}(\mu, R)=$ $R k_{F}(R)$ then $R$ exits the interval $\left[r_{-}(\ell), r_{+}(\ell)\right]$ (on either sides, see Fig. 2). 
As an example we consider the parametrization $V(r)=\mu v\left(r / r_{e}\right)$ where $v(\tilde{r})$ is dimensionless with $v(1)=1$. One defines $\lambda$ such that $\ell=\frac{1}{2} r_{e} \sqrt{2 \mu} \lambda$ and one has $r^{ \pm}(\ell)=r_{e} \tilde{r}^{ \pm}(\lambda)$ where $\tilde{r}^{ \pm}(\lambda)$ are the two roots of $\lambda=2 \tilde{r} \sqrt{1-v(\tilde{r})}$. One then obtains in the limit where $k_{F} r_{e}=r_{e} \sqrt{2 \mu} \gg 1$ at fixed ratio $\tilde{R}=R / r_{e}$ (i.e., in the bulk)

$$
\operatorname{Var} \mathcal{N}_{R} \simeq\left(\frac{k_{F} r_{e}}{2}\right)^{d-1}\left[A_{d}(\tilde{R}) \log \left(\frac{k_{F} r_{e}}{2}\right)+B_{d}(\tilde{R})+o(1)\right] \quad, \quad \tilde{R}=R / r_{e} \quad, \quad k_{F}=\sqrt{2 \mu}
$$

with

$$
\begin{aligned}
& A_{d}(\tilde{R})=\frac{1}{\pi^{2} \Gamma(d)}(2 \tilde{R} \sqrt{1-v(\tilde{R})})^{d-1} \quad, \quad B_{d}(\tilde{R})=\int_{0}^{2 \tilde{R} \sqrt{1-v(\tilde{R})}} \frac{d \lambda \lambda^{d-2}}{\pi^{2} \Gamma(d-1)}
\end{aligned}
$$

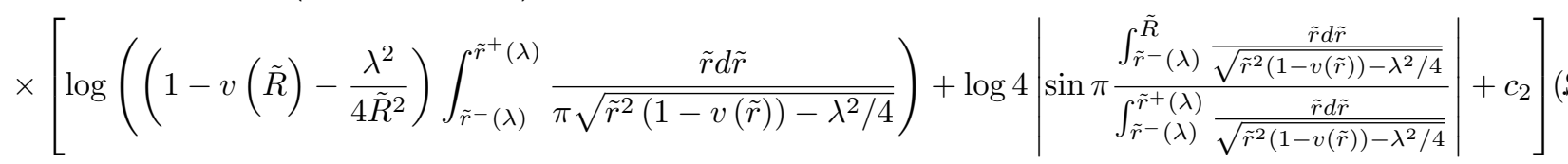

Asymptotics near the edge For $\tilde{R} \rightarrow 1$, i.e., as $R$ reaches the edge $r_{e}$, both $A_{d}$ and $B_{d}$ vanish. One has $v(\tilde{R}) \simeq$ $1-v^{\prime}(1)(1-\tilde{R})$, hence $A_{d}(\tilde{R}) \simeq \frac{2^{d-1}}{\pi^{2} \Gamma(d)}\left(v^{\prime}(1)(1-\tilde{R})\right)^{\frac{d-1}{2}}$. The upper bound in the integral in (S55) being small, one writes $\lambda=2\left(v^{\prime}(1)(1-\tilde{R})\right)^{1 / 2} u$, with $0<u<1$. In the integral in the numerator in the last term one can replace the bounds $\int_{\tilde{r}^{-}(\lambda)}^{\tilde{\tilde{R}}} \rightarrow \int_{\tilde{R}}^{r^{+}(\lambda)}$, and change variables to $\tilde{r}=1-(1-\tilde{R}) y$. The integral in the numerator becomes $\simeq\left(\frac{1-\tilde{R}}{v^{\prime}(1)}\right)^{1 / 2} \int_{u^{2}}^{1} \frac{d y}{\sqrt{y-u^{2}}}$. The argument of the sinus being small one can expand it and one finds that the integral $\int_{\tilde{r}^{-}(\lambda)}^{\tilde{r}^{+}(\lambda)}$ in the denominator exactly cancels the one in the first logarithm. One arrives at the asymptotics, for $\tilde{R} \rightarrow 1$

$$
B_{d}(\tilde{R}) \simeq \frac{2^{d-1}}{\pi^{2} \Gamma(d)}\left(v^{\prime}(1)(1-\tilde{R})\right)^{\frac{d-1}{2}}\left(\frac{3}{2} \log (1-\tilde{R})+\frac{1}{2} \log v^{\prime}(1)+c_{2}+3 \log 2+\frac{3}{2}(d-1) \int_{0}^{1} d u u^{d-2} \log \left(1-u^{2}\right)\right)
$$

Harmonic oscillator. We can now specify to the harmonic oscillator $V(r)=\frac{1}{2} r^{2}$, and we show that we recover the result (4) of the main text. One has $r_{e}=\sqrt{2 \mu}, v(\tilde{r})=\tilde{r}^{2}$ and $\tilde{r}^{ \pm}(\lambda)^{2}=\frac{1}{2}\left(1 \pm \sqrt{1-\lambda^{2}}\right)$. One thus immediately recovers the result for $A_{d}$ given in (5) in the text. To obtain the amplitiude $B_{d}$ we first compute the integral

$$
\int_{\tilde{r}^{-}(\lambda)}^{\tilde{R}} \frac{\tilde{r} d \tilde{r}}{\sqrt{\tilde{r}^{2}-\tilde{r}^{4}-\lambda^{2} / 4}}=\frac{1}{2} \phi(\tilde{R}) \quad, \quad \phi(\tilde{R})=\frac{\pi}{2}-\arctan \frac{1-2 \tilde{R}^{2}}{2 \sqrt{\tilde{R}^{2}-\tilde{R}^{4}-\lambda^{2} / 4}}
$$

which equals $\pi / 2$ for $\tilde{R}=r^{+}(\lambda)$. Hence the last logarithm in (S55) becomes $\log 4|\sin \phi(R)|=3 \log 2+\frac{1}{2} \log \left(R^{2}-\right.$ $\left.R^{4}-\lambda^{2} / 4\right)-\frac{1}{2} \log \left(1-\lambda^{2}\right)$ and we arrive at

$$
B_{d}(\tilde{R})=\int_{0}^{2 \tilde{R} \sqrt{1-\tilde{R}^{2}}} \frac{d \lambda \lambda^{d-2}}{\pi^{2} \Gamma(d-1)}\left[\log \left(4 \tilde{R} \frac{\left(1-\tilde{R}^{2}-\frac{\lambda^{2}}{4 \tilde{R}^{2}}\right)^{3 / 2}}{\left(1-\lambda^{2}\right)^{1 / 2}}\right)+c_{2}\right]
$$

This calculation is equivalent to the one sketched in the text in (25) where we used the result (21) in the text for the variance $\operatorname{Var} \mathcal{N}_{[0, R]}^{\mathrm{LUE}}$ associated the $1 \mathrm{~d}$ inverse square potential, related the LUE.

Integrating over $\lambda$, using the identity $(d-1) \int_{0}^{1} d \lambda \lambda^{d-2} \log \left(1-z \lambda^{2}\right)=z \Phi\left(z, 1, \frac{d+1}{2}\right)+\log (1-z)$, where $\Phi(z, s, a)$ is the function Lerch transcendant, defined as $\Phi(z, s, a)=\sum_{k \geq 0} \frac{z^{k}}{(k+a)^{s}}$ we obtain an explicit expression for $B_{d}$ valid in any dimension $d \geq 1$ [116]

$$
\begin{aligned}
B_{d}(\tilde{R}) & =\frac{1}{\pi^{2} \Gamma(d)}\left(2 \tilde{R} \sqrt{1-\tilde{R}^{2}}\right)^{d-1}\left[\log \left(2 \tilde{R} \sqrt{1-\tilde{R}^{2}}\right)+1-\frac{\gamma_{E}}{2}+2 \log 2-\frac{3}{2} \psi^{(0)}\left(\frac{d+1}{2}\right)\right. \\
& \left.+\log \left(1-\tilde{R}^{2}\right)-2 \tilde{R}^{2}\left(1-\tilde{R}^{2}\right) \Phi\left(4 \tilde{R}^{2}\left(1-\tilde{R}^{2}\right), 1, \frac{d+1}{2}\right)-\frac{1}{2} \log \left(1-4 \tilde{R}^{2}\left(1-\tilde{R}^{2}\right)\right)\right]
\end{aligned}
$$


In the limit $\tilde{R} \rightarrow 0$ it recovers the free fermion result (S40) using $k_{F} R=2 \mu \tilde{R}$. Specifying the formula (S60) to $d=2,3$ one finds

$$
2 \pi^{2} B_{2}(x)=2 x \sqrt{1-x^{2}}\left\{\log \left[\left(\frac{64 x}{1-2 x^{2}}\right)^{2}\left(1-x^{2}\right)^{3}\right]+2 \gamma_{E}-2\right\}+\log \left(\frac{\left|1-2 x \sqrt{1-x^{2}}\right|}{1+2 x \sqrt{1-x^{2}}}\right)
$$

and

$$
2 \pi^{2} B_{3}(x)=\left(1-2 x^{2}\right)^{2} \log \left|1-2 x^{2}\right|+4 x^{2}\left(1-x^{2}\right)\left\{\log \left[8 x\left(1-x^{2}\right)^{3 / 2}\right]+\gamma_{E}\right\}
$$

which are also given in (26) and (27) in the main text. The functions $A_{d}(x)$ and $B_{d}(x)$ for $d=2,3$ are plotted in Fig. 3. The function $B_{2}(x)$ was compared with simulations in Fig. 1 in the text. These functions have a non trivial behavior with a maximum and a minimum. Near the edge, for $r=r_{e}$, i.e $x=\tilde{R}=1$, the functions $B_{2}(x)$ and $B_{3}(x)$ vanish as

$$
\begin{aligned}
& B_{2}(x)=\frac{1}{\pi^{2}} \sqrt{2-2 x}\left(3 \log (1-x)+15 \log 2+2 \gamma_{E}-4\right)+O\left((1-x)^{3 / 2}\right), \\
& B_{3}(x)=\frac{2}{\pi^{2}}(1-x)\left(3 \log (1-x)+2 \gamma_{E}-1+9 \log 2\right)+O\left((x-1)^{2}\right)
\end{aligned}
$$

in agreement with the general formula (S57) using $v^{\prime}(1)=2$.



FIG. 3. The functions $A_{2}(x), A_{3}(x), B_{2}(x), B_{3}(x)$ (solid, dashed, dotted and dot-dashed lines respectively), that give the leading and subleading order terms of the variance of the number of particles inside a sphere for the harmonic oscillator for $d=2$ and $d=3$, see Eqs. (4), (5), (26) and (27) in the main text.

Remark. One can similarly calculate the variance of the number of fermions in a spherical shell $R_{1}<r<R_{2}$ from Eq. (2) in the text upon similar substitutions as described above (e.g. $a \rightarrow R_{1}, b \rightarrow R_{2}$ and so on).

Other types of potentials. Suppose now that the function $r \rightarrow r k_{F}(r)=r \sqrt{2(\mu-V(r))}$ grows monotonically from 0 to $+\infty$ for $r \in[0,+\infty[$. This is the case e.g. for a bounded and decreasing potential (non-confining). In that case there is a single turning point for $V_{\ell}(r)$ for any $\ell$, i.e., a single root $r^{-}(\ell)$ to $r k_{F}(r)=\ell$ and the situation is closer to the one for free fermions (in which case $k_{F}(r)=\sqrt{2 \mu}$ ). Then we can repeat the above derivation using now the $1 \mathrm{~d}$ formula (S28) and perform the same substitutions as above. Equivalently we can take the formal limit $r^{+}(\ell) \rightarrow+\infty$ in the $d$ dimensional formula (S53). This leads to the simpler formula

$$
\operatorname{Var} \mathcal{N}_{R} \simeq \frac{1}{2 \pi^{2}} \frac{2}{\Gamma(d-1)} \int_{0}^{R k_{F}(R)} d \ell \ell^{d-2}\left\{\log \left(\left[\left(R k_{F}(R)\right)^{2}-\ell^{2}\right] \int_{r^{-}(\ell)}^{R} \frac{r d r}{R^{2} \sqrt{r^{2} k_{F}(r)^{2}-\ell^{2}}}\right)+c_{2}+\log 2\right\}
$$

which reduces to the free fermion result $(\mathrm{S} 40)$ when $k_{F}(r)=\sqrt{2 \mu}$.

There are other possible situations depending on the form of $V(r)$, and an exhaustive discussion goes beyond this Letter. For instance for each $\ell$ there could be various number of roots (i.e., turning points), $r^{\alpha}(\ell)$ to the equation $r k_{F}(r)=\ell$, with multiple interval supports $I_{q}(\ell)=\left[r^{(2 q-1)}(\ell), r^{(2 q)}(\ell)\right], q=1,2, \ldots$ For a given $R$ only the interval containing $R$ contributes to the variance, since the other intervals are either full or empty of fermions. 


\section{EDGE BEHAVIOR AND MATCHING WITH THE BULK, IN $d=1$ AND $d>1$}

Here we give some more details about the matching of the number variance formulae obtained in the bulk, when entering the region of the edge of the Fermi gas.

We start with $d=1$ and the discussion below (20) in the text, and we focus on the inverse square potential, $V(x)=\frac{x^{2}}{2}+\frac{\alpha(\alpha-1)}{2 x^{2}}$ which corresponds to the LUE. For any smooth confining potential, as discussed in [11], for $x, y$ near the right edge $x^{+}$(and similarly for $x^{-}$) the kernel takes the universal scaling form $K_{\mu}(x, y) \simeq \frac{1}{w_{N}} K_{\mathrm{Ai}}\left(\frac{x-x^{+}}{w_{N}}, \frac{y-x^{+}}{w_{N}}\right.$ ) where $w_{N}$ is the width of the edge region $w_{N}=\left[2 V^{\prime}\left(x^{+}\right)\right]^{-1 / 3}$. Here $K_{\mathrm{Ai}}$ is the Airy kernel given by $K_{\mathrm{Ai}}(x, y)=$ $\frac{\operatorname{Ai}(x) \mathrm{Ai}^{\prime}(y)-\mathrm{Ai}^{\prime}(x) \mathrm{Ai}(y)}{x-y}$. Using this scaling form one obtains, for an arbitrary smooth potential and $a$ in the edge region, as was done in $[20,21]$ for the case of the harmonic oscillator/GUE,

$$
\operatorname{Var} \mathcal{N}_{[0, a]}=\int_{a}^{+\infty} d x \int_{0}^{a} d y K_{\mu}^{2}(x, y) \simeq \frac{1}{2} \mathcal{V}_{2}\left(\frac{a-x^{+}}{w_{N}}\right) \quad, \quad \mathcal{V}_{2}(\hat{a}):=2 \int_{\hat{a}}^{+\infty} d u \int_{-\infty}^{\hat{a}} d v K_{\mathrm{Ai}}^{2}(u, v)
$$

where the scaling function $\mathcal{V}_{2}(\hat{a})$, defined in [20,21], is universal. For a general potential the scaling variable is defined as $\hat{a}=\frac{a-x^{+}}{w_{N}}$ and (S66) holds for $\hat{a}=O(1)$. We expect that the limit $\hat{a} \rightarrow-\infty$ of the edge result (S66) should match with the limit $a \rightarrow x^{+}$of $(20)$ in the text, as we now check explicitly. In the limit $a \rightarrow x^{+}, a<x^{+}$, one has $k_{F}(a) \simeq \sqrt{2\left(x^{+}-a\right) V^{\prime}\left(x^{+}\right)}$hence $\int_{a}^{x^{+}} \frac{d z}{k_{F}(z)} \simeq \frac{\sqrt{2\left(x^{+}-a\right)}}{V^{\prime}\left(x^{+}\right)^{1 / 2}}$. Evaluating the terms in (20) in the text we have $\frac{1}{d \mu / d N} \sin \theta_{a} \simeq \frac{\pi}{d \mu / d N} \frac{\int_{a}^{x^{+}} \frac{d z}{k_{F}(z)}}{\int_{x^{-}}^{x^{-}} \frac{d z}{k_{F}(z)}} \simeq \frac{1}{V^{\prime}\left(x^{+}\right)^{1 / 2}} \sqrt{2\left(x^{+}-a\right)}$ which leads to the asymptotic behavior for $a \rightarrow x^{+}$coming from the bulk

$$
\operatorname{Var} \mathcal{N}_{[0, a]} \simeq \frac{1}{2 \pi^{2}}\left[c_{2}+\log \left(4\left(x^{+}-a\right)^{3 / 2}\left(2 V^{\prime}\left(x^{+}\right)\right)^{1 / 2}\right)\right]=\frac{1}{2 \pi^{2}}\left[\frac{3}{2} \log (-\hat{a})+c_{2}+2 \log 2\right]
$$

which can thus be nicely recast as a function of the edge scaling variable $\hat{a}$. Our result (S67) can be compared with the known results for $\frac{1}{2} \mathcal{V}_{2}(\hat{a})$ for $\hat{a} \rightarrow-\infty$. The leading order (the logarithm) agrees with the known result for the HO/GUE obtained in $[20,77]$. The $O(1)$ constant can further be extracted from the mathematical work of Bothner and Buckingham using Riemann Hilbert techniques in [76] (from the coefficient of $v^{2} / 2$ in the Taylor expansion in $v$ of their Eq. 1.11 with their $s \equiv \hat{a}$ ). Here we have obtained this asymptotics by a completely different method and both results agree. Finally, as stated in the text, one can show that when $a$ is fixed in the bulk and $b$ reaches the edge, the heights at these locations decorelate and $H(a, b)=o(1)$, equivalently from Eq. (9) in the main text, $\operatorname{Var}\left(\mathcal{N}_{[a, b]}\right) \simeq \operatorname{Var}\left(\mathcal{N}_{[a, \infty[}\right)+\operatorname{Var}\left(\mathcal{N}_{[b, \infty[}\right)$

For a confining central potential $V(r)$ in dimension $d$, we use again (23) in the text for $p=2$, substituting the $d=1$ edge scaling form discussed above for each angular momentum sector $\ell$ associated to $m_{\ell}$ fermions in the potential $V_{\ell}$, hence

$$
\left\langle\mathcal{N}_{R}^{2}\right\rangle^{c}=\sum_{\ell \geq 0}^{\ell_{\max }(\mu)} g_{d}(\ell)\left\langle\mathcal{N}_{R}^{2}\right\rangle_{\ell}^{c} \simeq \sum_{\ell \geq 0}^{\ell_{\max }(\mu)} \frac{2 \ell^{d-2}}{\Gamma(d-1)} \frac{1}{2} \mathcal{V}_{2}\left(\frac{R-r^{+}(\ell)}{w_{m_{\ell}}}\right)
$$

where $w_{m_{\ell}}=\left[2 V_{\ell}^{\prime}\left(r^{+}(\ell)\right)\right]^{-1 / 3}=\left[2 V^{\prime}\left(r^{+}(\ell)\right)-\frac{\ell^{2}}{r^{+}(\ell)^{3}}\right]^{-1 / 3}$. By definition $r^{+}(\ell)$ is solution of $($ for $\ell \gg 1)$

$$
V\left(r^{+}(\ell)\right)-V\left(r_{e}\right)+\frac{\ell^{2}}{2 r^{+}(\ell)^{2}}=0
$$

One can tentatively expand in powers of $\ell$, recalling that $r^{+}(\ell \simeq 0)=r_{e}$

$$
r^{+}(\ell)-r_{e}=-\frac{\ell^{2}}{2 r_{e}^{2} V^{\prime}\left(r_{e}\right)}-\frac{4 V^{\prime}\left(r_{e}\right)+r_{e} V^{\prime \prime}\left(r_{e}\right)}{8 r_{e}^{5} V^{\prime}\left(r_{e}\right)^{3}} \ell^{4}+O\left(\ell^{6}\right)=-w_{N}^{3} \frac{\ell^{2}}{r_{e}^{2}}+O\left(\frac{w_{N}^{6}}{r_{e}^{5}} \ell^{4}\right)
$$

where we introduced $w_{N}=\left(2 V^{\prime}\left(r_{e}\right)\right)^{-1 / 3}$ the width of the edge region in any dimension (for a central potential) [11]. It is natural to expect (and consistent as we find below) that the values of $\ell$ which contribute most in the sum in (S68) are such that $r^{+}(\ell)-r_{e}=O\left(w_{N}\right)$. From the first term in the expansion in (S70), we see that they are of order $\ell=O\left(r_{e} / w_{N}\right)$. It is consistent since the subleading term $O\left(\ell^{4}\right)$ is then smaller than the $O\left(\ell^{2}\right)$ term by a factor $w_{N} / r_{e}$ 
(and similarly for the higher orders). Furthermore, one can check that replacing $w_{m_{\ell}}$ by $w_{N}$ in the scaling variable in (S68), for $\ell=O\left(r_{e} / w_{N}\right)$, also amounts to neglecting subdominant terms. Hence the scaling variable in (S68) can be replaced as $\frac{R-r^{+}(\ell)}{w_{m_{\ell}}} \simeq \frac{1}{w_{N}}\left(R-r_{e}+w_{N}^{3} \frac{\ell^{2}}{r_{e}^{2}}\right)$ and we obtain

$$
\left\langle\mathcal{N}_{R}^{2}\right\rangle^{c} \simeq \int_{0}^{\ell_{\max }(\mu)} \frac{2 \ell^{d-2} d \ell}{\Gamma(d-1)} \frac{1}{2} \mathcal{V}_{2}\left(\hat{R}+w_{N}^{2} \frac{\ell^{2}}{r_{e}^{2}}\right) \simeq\left(\frac{r_{e}}{w_{N}}\right)^{d-1} \int_{0}^{\infty} \frac{d \xi \xi^{\frac{d-3}{2}}}{2 \Gamma(d-1)} \mathcal{V}_{2}(\hat{R}+\xi) \quad, \quad \hat{R}=\frac{R-r_{e}}{w_{N}}
$$

upon introducing the $O(1)$ variable $\xi=\left(w_{N} \ell / r_{e}\right)^{2}$, which is the formula (28) in the text. Note that we have also obtained this formula by a direct calculation [98], without using the decoupling in (23) in the text, but using instead the exact form of the edge kernel in dimension $d$ obtained in [11]. The formula (S71) is compared with numerical simulations in $d=2$ in Fig. 6 in Section XI.

To see how this formula matches with our result for the bulk in dimension $d$, we now evaluate (S71) in the limit $\hat{R} \rightarrow-\infty$, inserting the asymptotics (S67) of $\frac{1}{2} \mathcal{V}_{2}(\hat{a})$ for large negative $\hat{a}$. The integral over $\xi$ in (S71) can be splitted into a first part $\int_{0}^{-\hat{R}} d \xi$ and a second part $\int_{-\hat{R}}^{+\infty} d \xi=\int_{0}^{+\infty} d v$ writing $\xi=-\hat{R}+v$. Since $\mathcal{V}_{2}(v)$ decays fast at $v \rightarrow+\infty$, this second part is $O\left((-\hat{R})^{\frac{d-3}{2}}\right)$ which is subdominant. In the first part we write $\xi=-\hat{R} u^{2}$ and obtain for $\hat{R} \rightarrow-\infty$

$$
\left\langle\mathcal{N}_{R}^{2}\right\rangle^{c} \simeq\left(\frac{r_{e}}{w_{N}}\right)^{d-1} \frac{(-\hat{R})^{\frac{d-1}{2}}}{\pi^{2} \Gamma(d)}\left[\frac{3}{2} \log (-\hat{R})+c_{2}+2 \log 2+\frac{3}{2}(d-1) \int_{0}^{1} d u u^{d-2} \log \left(1-u^{2}\right)\right]
$$

Upon using that $-\hat{R}=(1-\tilde{R}) r_{e} / w_{N}$ with $w_{N}=\left[r_{e} /\left(2 \mu v^{\prime}(1)\right]^{1 / 3}\right.$ one can check that (S72) agrees with the result (S54) and the asymptotics for $A_{d}$ and $B_{d}$ given below (S55) and in (S57) for a general smooth central potential of the form $V(r)=\mu v\left(r / r_{e}\right)$. This shows that our results for the variance in the bulk and at the edge match smoothly in any $d$.

Finally, different universality classes occur in the case of "hard edges". For instance in $d=1$, the kernels for the hard box near $x=0$ or $x=L$, or for the inverse square well near $x=x^{-}$, take universal scaling forms at a distance $1 / k_{F}$ near the edge, studied in [81]. We checked that our bulk results match these known cases in $d=1$, when $a \rightarrow 0$.

\section{HIGHER CUMULANTS AND ENTROPY}

\section{A. Higher cumulants}

In $d=1$, for the specific potentials and geometries related to RMT described in Section II the higher cumulants of the number of fermions $\mathcal{N}_{\mathcal{I}}$ in any interval $\mathcal{I}$ can be extracted from known results in random matrix theory, using the mapping between the fermion positions $x_{i}$ and the eigenvalues $\lambda_{i}$. In RMT the generating function of the cumulants is computed from Fisher-Hartwig type asymptotics of Hankel and Toeplitz determinants, using Riemann Hilbert methods [17, 22, 58, 82, 89]. Let us focus on Ref. [89] where the GUE, LUE and JUE are studied from which we obtain the results for the $\mathrm{HO}$, inverse square well and the Jacobi box respectively.

Using standard methods for determinantal processes, such as the Cauchy Binet formula, the generating function of the cumulants of $\mathcal{N}_{\mathcal{I}}$ for the $\mathrm{HO} / \mathrm{GUE}$ with $V(x)=\frac{1}{2} x^{2}$ (where $x_{i}=\lambda_{i}$ ) is given by the Hankel determinant

$$
\left\langle e^{-s \mathcal{N}_{\mathcal{I}}}\right\rangle=\left\langle\prod_{i=1}^{N}\left(1-\left(1-e^{-s}\right) \chi_{\mathcal{I}}\left(x_{i}\right)\right)\right\rangle=\frac{\operatorname{det}_{0 \leq k, l \leq N-1}\left(\int_{-\infty}^{\infty} e^{-x^{2}} x^{k+l}\left(1-\left(1-e^{-s}\right) \chi_{\mathcal{I}}(x)\right) d x\right)}{\operatorname{det}_{0 \leq k, l \leq N-1}\left(\int_{-\infty}^{\infty} e^{-x^{2}} x^{k+l} d x\right)}=\frac{G_{N}(\overrightarrow{0}, \vec{\beta}, V, 0)}{G_{N}(\overrightarrow{0}, \overrightarrow{0}, V, 0)}
$$

where $\chi_{\mathcal{I}}(x)$ is the indicator function of the interval $\mathcal{I}$, i.e., $\chi_{\mathcal{I}}(x)=1$ if $x \in \mathcal{I}$ and $\chi_{\mathcal{I}}(x)=0$ otherwise. The last identity connects to the notations in Ref. [89], where $V$ denotes the potential $V(x)=2 x^{2}$ in $(1.1)-(1.2)$ there, with $W=0$ and $\psi(x)=2 / \pi$, and the charges $\alpha_{i}$ in (1.6) there are all zero for the GUE. The choice of the $m$ non zero parameters $\vec{\beta}$ entering (1.6)-(1.7) depends on the type of interval $\mathcal{I}$. For $\mathcal{I}=[a, b]$ with $a=\tilde{a} \sqrt{2 N}$ and $b=\tilde{b} \sqrt{2 N}$ (where we are interested in $-1<\tilde{a} \neq \tilde{b}<1$ in the bulk) the choice is $\vec{\beta}=\left(\beta_{1}=p /(2 i \pi), \beta_{2}=-s /(2 i \pi)\right), t_{1}=\tilde{a}$ and $t_{2}=\tilde{b}$, so that from (1.7) there, $\omega(x)=1$ outside the interval $\left[t_{1}=\tilde{a}, t_{2}=\tilde{b}\right]$ and $e^{-s}$ inside. For the semi-infinite interval $\mathcal{I}=[-\infty, a]$ one must choose $\vec{\beta}=\left(\beta_{1}=s /(2 i \pi)\right)$ and $t_{1}=\tilde{a}$. The case of a collection of intervals is similarly obtained considering $m \geq 3$. Using the Theorem 1.1 in Ref. [89] one obtains for large $N$, up to terms of order $O(\log N / N)$

$$
\log \left\langle e^{-s \mathcal{N}_{\mathcal{I}}}\right\rangle=\sum_{j=1}^{m} \log G\left(1+\beta_{j}\right) G\left(1-\beta_{j}\right)+\ldots= \begin{cases}2 \log G\left(1+\frac{i s}{2 \pi}\right) G\left(1-\frac{i s}{2 \pi}\right)+\ldots \quad, \quad \mathcal{I}=[a, b] \\ \log G\left(1+\frac{i s}{2 \pi}\right) G\left(1-\frac{i s}{2 \pi}\right)+\ldots, \quad, \quad \mathcal{I}=[-\infty, a]\end{cases}
$$


where $\ldots$ denote terms proportional to $\beta_{j}$ and $\beta_{j}^{2}$, i.e., to $s, s^{2}$. These terms have been discussed above and reproduce respectively the first and and second cumulant of $\mathcal{N}_{\mathcal{I}}$. Expanding the Barnes function in (S74) in powers of $s$ one obtains the formula (29) of the text for the higher order cumulants of $\mathcal{N}_{[a, b]}$ for the harmonic oscillator, with $a, b$ in the bulk on macroscopic scales. The odd cumulants are zero and the $2 n$-th cumulants are given by $\kappa_{2 n}$ defined in (29) in the text, which, remarkably is independent of $a, b$. The higher cumulants of $\mathcal{N}_{[-\infty, a]}$ are smaller by a factor $1 / 2$.

For the inverse square potential $V(x)=\frac{x^{2}}{2}+\frac{\alpha(\alpha-1)}{2 x^{2}}$ we can use Theorem 1.2 of Ref. [89] ( $G_{N}$ is now denoted $\left.L_{N}\right)$ with $V(x)=2(x+1)$ in (1.1)-(1.2) there, with $W=0$ and $\psi(x)=1 / \pi$, and the charges $\alpha_{i}$ in (1.6) all zero except $\alpha_{0}=\alpha-\frac{1}{2}$. The parameter $\beta_{i}$ with $1 \leq i \leq m$ are the same as in the previous case. For the interval $\mathcal{I}=[2 \sqrt{N} \tilde{a}, 2 \sqrt{N} \tilde{b}]$ one has $t_{1}=2 \tilde{a}^{2}-1$ and $t_{2}=2 \tilde{b}^{2}-1$. One sees that the result (S74), leading to (29) of the text also holds for the inverse square potential. Note that the analog of the semi-infinite interval $]-\infty, a]$ is now $[0, a]$. From Theorem 1.3 of Ref. [89] we see that the same result holds for the Jacobi box potential. Finally the same result holds for the higher cumulants for $\mathcal{N}_{[a, b]}$ for the CUE, hence for the free fermions on the circle for which the generating function can be written as a Toeplitz determinant $[6,17,104]$.

Furthermore the same result holds for microscopic scales, e.g. for an interval $[a, b]$ with $|a-b| \ll 1$, in the limit where $|a-b| k_{F}(a)$ is fixed but large, see Theorem 1.1 of Ref. [22] as is shown from similar analysis using the sine-kernel. This leads to the conjecture given in the text that it arises from microscopic scales and holds for an arbitrary smooth potential $V(x)$.

In space dimension $d>1$, for a central potential $V(r)$ and a spherical domain $\mathcal{D}$ of radius $R$, the formula (23) of the text determines the $p$-th order cumulant of $\mathcal{N}_{\mathcal{D}}$ from the knowledge of the $p$-th order cumulant of $\mathcal{N}_{[0, R]}$ for the set of 1 d potentials $V_{\ell}(r)=V(r)+\frac{\alpha(\alpha-1)}{2 r^{2}}$ on $[0, r]$ with $\alpha=\ell+\frac{d-1}{2}$, where $\ell$ labels the angular momentum sector. In the large $\mu$ limit, we know from Section VII that this sum is dominated by values of $\ell \gg 1$, such that $g_{d}(\ell) \simeq \frac{2 \ell^{d-2}}{\Gamma(d-1)}$.

Let us start with free fermions $V(r)=0$. In that case one needs the higher cumulants for $V_{\ell}(r) \simeq \frac{\ell^{2}}{2 r^{2}}$ for $\ell \gg 1$. These are known from a very recent work in mathematics [84] on the Bessel process, which yields again the same formula (S74), recalling that $[0, R]$ corresponds to the second line in this formula, hence leading to $1 / 2$ times the result in (29) of the text. Using (23) in the text we obtain, for $k_{F} R \gg 1$ and $n \geq 2$

$$
\left\langle\mathcal{N}_{R}^{2 n}\right\rangle^{c} \simeq \frac{2}{\Gamma(d-1)} \int_{0}^{k_{F} R} d \ell \ell^{d-2} \times \frac{1}{2} \kappa_{2 n}=\frac{\left(k_{F} R\right)^{d-1}}{\Gamma(d)}\left(\kappa_{2 n}+o(1)\right) \quad, \quad k_{F}=\sqrt{2 \mu}
$$

where the integration upper bound comes from the constraint $V_{\ell}(r) \simeq \frac{\ell^{2}}{2 r^{2}} \leq \mu$. Note that since the result of [84] is rigorous, our formula for the higher cumulants for free fermions is expected to be exact independently of the conjecture stated above and in the main text.

For the harmonic oscillator $V(r)=\frac{1}{2} r^{2}$, it turns out that the result for the higher cumulants discussed above for the LUE holds also for large $\ell=O(\mu)$ [92]. Therefore, in this case, we arrive at the following formula

$$
\left\langle\mathcal{N}_{R}^{2 n}\right\rangle^{c}=\frac{\left(k_{F}(R) R\right)^{d-1}}{\Gamma(d)}\left(\kappa_{2 n}+o(1)\right) \quad, \quad k_{F}(R)=\sqrt{2(\mu-V(R))}
$$

since the upper bound in the integral in (S75) is now $\ell=\ell_{c}(\mu, R) \simeq k_{F}(R) R$, from the constraint that $V_{\ell}(r) \simeq$ $V(r)+\frac{\ell^{2}}{2 r^{2}} \leq \mu$. In the text below Eq. (30) in the main text, we conjecture that this result in Eq. (S76), which is close to being rigorous for $V(r)=\frac{1}{2} r^{2}$, holds for a more general smooth central potential.

\section{B. Entanglement entropy}

We now apply our results to the calculation of the bipartite Rényi entanglement entropy of a $d$-dimensional domain $\mathcal{D}$ with its complement $\overline{\mathcal{D}}$. It is defined for $q \geq 0$ as $S_{q}(\mathcal{D})=\frac{1}{1-q} \ln \operatorname{Tr}\left[\rho_{\mathcal{D}}^{q}\right]$, where $\rho_{\mathcal{D}}=\operatorname{Tr}_{\overline{\mathcal{D}}}[\rho]$ is obtained by tracing out the density matrix $\rho$ of the system over $\overline{\mathcal{D}}$. For noninteracting fermions [24-27] the entropy can be expressed in any dimension $d$ as a series involving the cumulants of $\mathcal{N}_{\mathcal{D}}$

$$
S_{q}(\mathcal{D})=\frac{\pi^{2}}{6}\left(1+\frac{1}{q}\right) \operatorname{Var} \mathcal{N}_{\mathcal{D}}+\sum_{n \geq 2} s_{n}^{(q)}\left\langle\mathcal{N}_{\mathcal{D}}^{2 n}\right\rangle^{c} \quad, \quad s_{n}^{(q)}=\frac{(-1)^{n}(2 \pi)^{2 n} 2 \zeta\left(-2 n, \frac{1+q}{2}\right)}{(q-1) q^{2 n}(2 n) !}
$$

where the coefficient $s_{n}^{(q)}$ are given in $[27]$ and $\zeta(s, a)=\sum_{k=0}^{\infty}(k+a)^{-s}$ is the generalized Riemann zeta function. An immediate consequence of this property, together with $(23)$ of the text, is that, for any central potential $V(r)$ and any 
domain $\mathcal{D}$ with spherical symmetry in dimension $d$, the entropy can be written as a sum of entropies of corresponding one-dimensional domains associated to an angular momentum sector. For instance, for $\mathcal{D}=\mathcal{B}_{R}$ the sphere of radius $R$ centered at the origin, one has

$$
S_{q}(\mathcal{D})=\sum_{\ell=0}^{\ell_{\max }(\mu)} g_{d}(\ell) S_{q}^{\ell}([0, R])
$$

where $S_{q}^{\ell}([0, R])$ is the bipartite entanglement entropy of the interval $[0, R]$ for the 1 d problem with potential $V_{\ell}(r)$ for $r \in \mathbb{R}^{+}$. Although (S78) can be useful, we will instead directly substitute our result (S76) for the cumulants in dimension $d$ into (S77), leading to

$$
S_{q}\left(\mathcal{B}_{R}\right)=\frac{\pi^{2}}{6}\left(1+\frac{1}{q}\right) \operatorname{Var}_{R}+\frac{\left(k_{F}(R) R\right)^{d-1}}{\Gamma(d)} \sum_{n \geq 2} s_{n}^{(q)}\left(\kappa_{2 n}+o(1)\right)
$$

which is valid for any central potential $V(r)$, with $k_{F}(R)=\sqrt{2(\mu-V(R))}$, in the limit where $R k_{F}(R) \gg 1$. The analogous formula in $d=1$ for the interval $[a, b]$ and a smooth potential $V(x)$ is, in the limit of large $\mu$

$$
S_{q}([a, b])=\frac{\pi^{2}}{6}\left(1+\frac{1}{q}\right) \operatorname{Var}_{[a, b]}+\sum_{n \geq 2} s_{n}^{(q)}\left(\kappa_{2 n}+o(1)\right) .
$$

Note that in the case of a semi-infinite interval $[c, a]$ with $c=-\infty, c=0$, for fermions on $\mathbb{R}^{\text {and }} \mathbb{R}^{+}$respectively, there is a factor $1 / 2$ in front of the last sum.

To evaluate the sum $\sum_{n>2} s_{n}^{(q)} \kappa_{2 n}$, we take advantage of the fact that it is independent both of the potential and the dimension $d$, and is already known for free fermions in $d=1$. In $d=1$ it is well known that the entanglement entropy of free fermions for an interval $\mathcal{D}=[a, b]$ is given by (for $k_{F}|a-b| \gg 1$ )

$$
S_{q}^{\mathrm{ff}}([a, b])=\frac{q+1}{6 q} \log \left(2 k_{F}|a-b|\right)+E_{q}+o(1), E_{q}=\frac{q+1}{q} \int_{0}^{+\infty} \frac{d t}{t}\left[\frac{1}{1-q^{-2}}\left(\frac{1}{q \sinh (t / q)}-\frac{1}{\sinh t}\right) \frac{1}{\sinh t}-\frac{e^{-2 t}}{6}\right]
$$

with $k_{F}=\sqrt{2 \mu}$ and where $E_{q}$ was obtained in [36], see also Eq. (11) in [94]. Since we also know the variance of $\mathcal{N}_{[a, b]}$ for free fermions, $\operatorname{Var} \mathcal{N}_{[a, b]}=\frac{1}{\pi^{2}}\left(\log k_{F}|a-b|+c_{2}+o(1)\right)$, (see the text), we obtain that $\sum_{n \geq 2} s_{n}^{(q)} \kappa_{2 n}=\tilde{E}_{q}=$ $E_{q}-\frac{q+1}{6 q}\left(1+\gamma_{E}\right)$, using that $c_{2}=\log 2+1+\gamma_{E}$. This leads to the equation (34) given in the text

$$
S_{q}(\mathcal{D})=\frac{\pi^{2}}{6} \frac{q+1}{q} \operatorname{Var} \mathcal{N}_{\mathcal{D}}+\eta_{d} \frac{\left(k_{F}(R) R\right)^{d-1}}{\Gamma(d)}\left(\tilde{E}_{q}+o(1)\right)
$$

for $k_{F}(R) R \gg 1$, where we introduced the parameter $\eta_{d}$ to discuss the various cases. For a central potential in dimension $d>1$ and $\mathcal{D}=\mathcal{B}_{R}$ the sphere of radius $R$, Eq. (S82) holds with $\eta_{d}=1$, as a consequence of (S79). For a spherical shell $r \in\left[R_{1}, R_{2}\right]$, (S82) can be generalized by replacing $\left(k_{F}(R) R\right)^{d-1} \rightarrow 2\left(k_{F}\left(R_{1}\right) R_{1}\right)^{d-1}+\left[\left(k_{F}\left(R_{2}\right) R_{2}\right)^{d-1}-\left(k_{F}\left(R_{1}\right) R_{1}\right)^{d-1}\right]$ (i.e., using $\eta_{d}=2$ and $\eta_{d}=1$ for different $\ell$ ). In dimension $d=1$, if $\mathcal{D}=[a, b]$ where $a, b$ are both in the bulk, the Eq. (S82) holds in the limit of large $\mu$, with $\eta_{d}=1$, setting $d=1$ in the equation, i.e., replacing $\frac{\left(k_{F}(R) R\right)^{d-1}}{\Gamma(d)} \rightarrow 1$ in Eq. (S82). For the semi-infinite interval $[c, a]$ with $c=-\infty, c=0$, for fermions on $\mathbb{R}$ and $\mathbb{R}^{+}$respectively, one has $\eta_{d}=\frac{1}{2}$.

Remark: for $q=1$ there is a simpler formula which relates the generating function of the cumulants and the entanglement entropy [9], i.e., $S_{1}(\mathcal{D})=\frac{1}{4} \int_{-\infty}^{\infty} \frac{\ln \left\langle e^{-s \mathcal{N}_{\mathcal{D}}}\right\rangle}{\sinh ^{2}(s / 2)} d s$. This allows to obtain the previous results without considering explicitly the sum over the cumulants, using the results for the generation function, see Eq. (S74), and the identity $E_{1}=\frac{1}{2} \int_{-\infty}^{\infty} \ln \left[G\left(1+\frac{p}{2 i \pi}\right) G\left(1-\frac{p}{2 i \pi}\right)\right] \frac{1}{\sinh ^{2}(p / 2)} d p=0.495018 \ldots$

Our main result for the entanglement entropy is thus Eq. (S82). Combined with the explicit expressions for the variance that we have obtained in this paper, Eq. (S82) provides explicit expressions for the entropy in the limit of large $\mu$, or large $k_{F}(R) R$, in the following cases.

In $d=1$ for the potentials $V(x)$ related to RMT, the HO, the inverse square well, the Jacobi box, the formula (S82) is based only on the available rigorous results for all cumulants, see Section X A, and thus does not rely on any conjecture. The leading term $O(\log \mu)$ was known in previous works for the HO, and some formula were proposed for 
the $O(1)$ subleading term $[32,51,52]$. Our general formula leads to the following result for the entanglement entropy for the $\mathrm{HO}, V(x)=\frac{1}{2} x^{2}$, for any interval $[a, b]$ in the bulk in the large $N$ limit

$$
S_{q}([\tilde{a} \sqrt{2 N}, \tilde{b} \sqrt{2 N}])=\frac{q+1}{6 q}\left[\ln \mu+\frac{3}{4} \ln \left(1-\tilde{a}^{2}\right)\left(1-\tilde{b}^{2}\right)+\ln \frac{4|\tilde{a}-\tilde{b}|}{1-\tilde{a} \tilde{b}+\sqrt{\left(1-\tilde{a}^{2}\right)\left(1-\tilde{b}^{2}\right)}}+c_{2}\right]+\tilde{E}_{q}+o(1)
$$

with $\mu=N$, and we recall that $\tilde{E}_{q}=E_{q}-\frac{(q+1)\left(1+\gamma_{E}\right)}{6 q}$. Note that this formula is quite similar to Eq. (18) in [32] which however is only valid up to a $O(1)$ constant (independent of $\tilde{a}, \tilde{b}$ ), which is obtained here. We can obtain similar results for the semi-infinite interval of the $\mathrm{HO}$, and for the inverse square well. In the case of the hard box, i.e., $V(x)=0$ for $x \in[0, L]$ and Dirichlet boundary conditions in $x=0, L$ (which is related to the JUE), one can use the the formula (S82) together with the result obtained for the variance in Eq. (S26) to compute the entanglement entropy of the segment $[0, a]$, in which case $\eta_{d}=1 / 2$ as explained below Eq. (S82). One obtains, for large $\mu$ (equivalently for large $N)$

$$
S_{q}([0, a])=\frac{1}{12} \frac{q+1}{q} \ln \left[4 N \sin \left(\frac{\pi a}{L}\right)\right]+\frac{E_{q}}{2}+o(1)
$$

which coincides with the known result (see Eq. (65) in Ref. [94]).

In $d=1$ for an arbitrary smooth potential $V(x)$ the formula (S82) can be combined with our results (2) and (20) in the text for the variance to obtain the explicit expression of the entanglement entropy for an interval $[a, b]$ in the bulk, and a semi infinite interval. It relies on our conjecture of the universality of the higher order cumulants. In Ref. [32] some formula were also proposed based on a very different approach using field theory and did not attempt to determine the $O(1)$ terms.

In $d>1$ our formula (S82) for a spherical domain is exact for free fermions based on rigorous results in the math literature, see Section X A, and thus does not rely on any conjecture. The leading term $S_{q}(\mathcal{D}) \propto R^{d-1} \log R$ at large $R$ agrees with the Widom conjecture for a spherical domain [38-41, 43], proved in [97]. In addition, we obtain here the first correction $O\left(R^{d-1}\right)$.

In $d>1$ for a general central potential our formula (S82) for a spherical domain using our results for the variance, displayed in Section VIII, and (4) and (5) in the text for the HO, is completely new. It has the same validity as formula (S76) for the higher cumulants, i.e., it relies on two very natural conjectures detailed in Section X A.

\section{NUMERICAL CALCULATIONS}

In this Section we briefly discuss some of the methods to compute numerically the number variances such as those displayed in Fig. 1 of the main text and in the figures below. The first one amounts to evaluate numerically the double integral (S7) that gives the height field and the variance from (S3), which however is delicate because of the fast oscillations of the integrand. An alternative method, which we found to be more efficient in most cases, is to generate a set of realizations of the positions of the fermions drawn from the quantum JPDF $\left|\Psi_{0}\right|^{2}$, and compute the empirical variance from it. For some particular potentials, such realizations can be generated very efficiently using the known connections to RMT, see Section II. This is the method that we used to generate all of the numerical data presented in this work [117], except for the hard-box potential where a numerical evaluation of the integral (S7) was performed.

Consider the HO. In $d=1$, we generate the eigenvalues of a random $N \times N$ GUE matrix. In $d>1$, to compute the variance of the number of particles $\mathcal{N}_{R}$ inside a sphere of radius $R$, we generate realizations of the radial coordinates $r_{1}, \ldots, r_{N}$. By exploiting the decoupling properties between the different angular sectors discussed in Section VI, we generate samples of radial coordinates within each angular sector separately. This amounts to generate fermion positions in the effective $1 d$ potential $V_{\ell}(r)=\frac{r^{2}}{2}+\frac{\left(\ell+\frac{d-3}{2}\right)\left(\ell+\frac{d-1}{2}\right)}{2 r^{2}}$. This corresponds to the inverse square well with $\alpha=\ell+\frac{d-1}{2}$. This is conveniently done by exploiting the mapping to the eigenvalues of a random matrix from the LUE matrix (as described in Section II). We used the tridiagonal matrix representations of GUE and LUE matrices [103] in order to efficiently generate their eigenvalues.

We start by testing our numerical methods on two cases related to GUE and LUE which were studied previously in [20, 21]. In Fig. 4 we give our results for $\operatorname{Var}\left(\mathcal{N}_{[-a, a]}\right)$ and $\operatorname{Var}\left(\mathcal{N}_{[a, \infty[}\right)$ for $N=100$ fermions in a harmonic trap in $d=1$. Each of the data points was generated by simulating $5 \times 10^{4}$ GUE matrices. Similarly, in Fig. 5 (a) we plot results for $\operatorname{Var}\left(\mathcal{N}_{[0, a]}\right)$ for $N=100$ fermions in an inverse square potential with $\alpha=5 / 2$, where each of the data points was generated by simulating $5 \times 10^{4}$ LUE matrices with $N=100$ and $M=102$. Finally, in Fig. 5 (b) we plot results for $\operatorname{Var}\left(\mathcal{N}_{[0, a]}\right)$ for $N=100$ fermions in a hard-box potential on the interval [0,L], where a numerical 
evaluation of the integral (S7) was performed, using the explicitly known kernel [81]. In all cases, the data shows excellent agreement with our theoretical predictions, as described in the figures' captions.
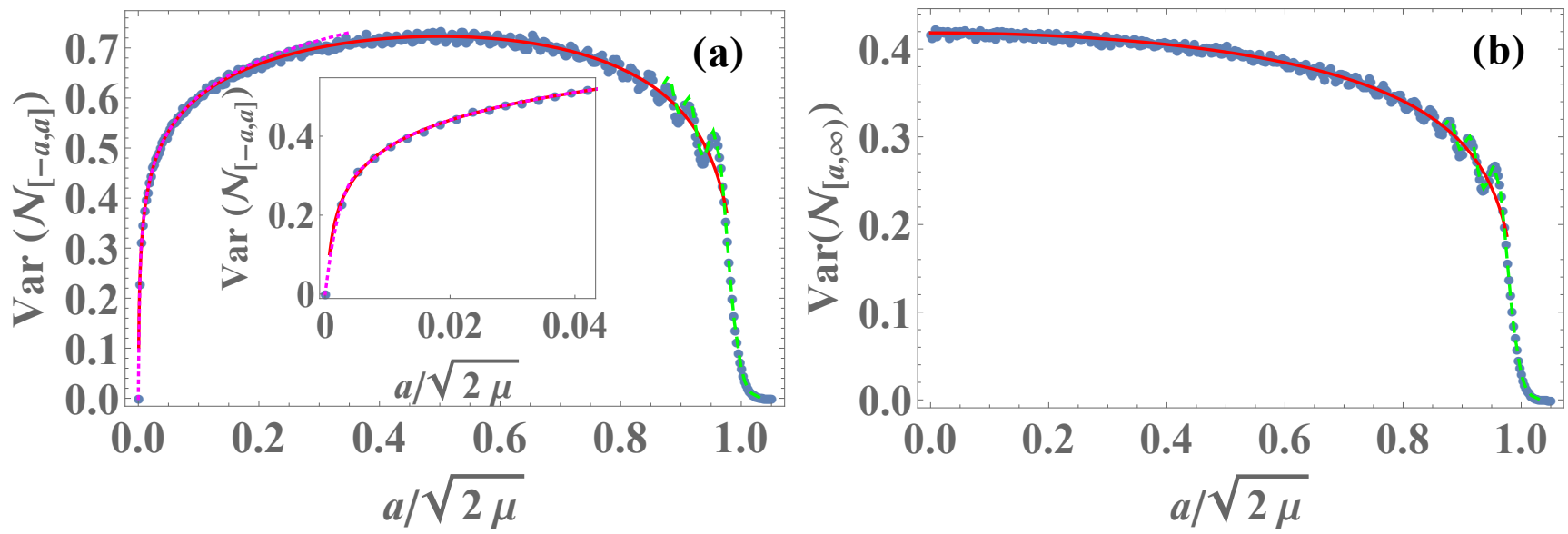

FIG. 4. Variance of $\mathcal{N}_{[-a, a]}$ (a) and of $\mathcal{N}_{[a, \infty)}$ (b) for the $1 d$ harmonic oscillator $V(x)=x^{2} / 2$, plotted vs $\tilde{a}=a / \sqrt{2 \mu}$ for $\mu=100$ (we recall that $\mu \simeq N$ ). The simulations (symbols) show excellent agreement with our predictions: In the bulk, with Eq. (S23) in (a) and Eq. (S22) in (b), and near the edge $\tilde{a}=1$, with the scaling form (S66). The dotted line in (a) (see the inset for a zoom on this region) is the prediction for the variance for a microscopic interval, $\pi^{2} \operatorname{Var} \mathcal{N}_{[-a, a]} \simeq U(0)-U\left(2 a k_{F}(0)\right)$, as described around Eq. (17) in the main text: it is expected to be valid in the regime $a \sim 1 / \sqrt{\mu}$, i.e., $\tilde{a} \sim 1 / \mu \ll 1$.

We now give some additional details for the $\mathrm{HO}$ in $d=2, V(r)=\frac{1}{2} r^{2}$, with $\mu=100$, corresponding to $N=$ $\mu(\mu+1) / 2=5050$. The numerical data that is plotted in Fig. 1 of the main text was computed over $2 \times 10^{6}$ realizations of sets $r_{1}, \ldots, r_{N}$ of the fermions' radial coordinates using the method that we described above. For clarity, we display in Fig. 6 (a) a close-up of the edge regime $\tilde{R} \simeq 1$ of Fig. 1 of the main text, showing excellent agreement between the numerical data and the theoretical edge prediction (28) in the text. Although we did not report it in Fig. 1 in the text we find excellent agreement for small values of $R$ with the free fermion result (S40).
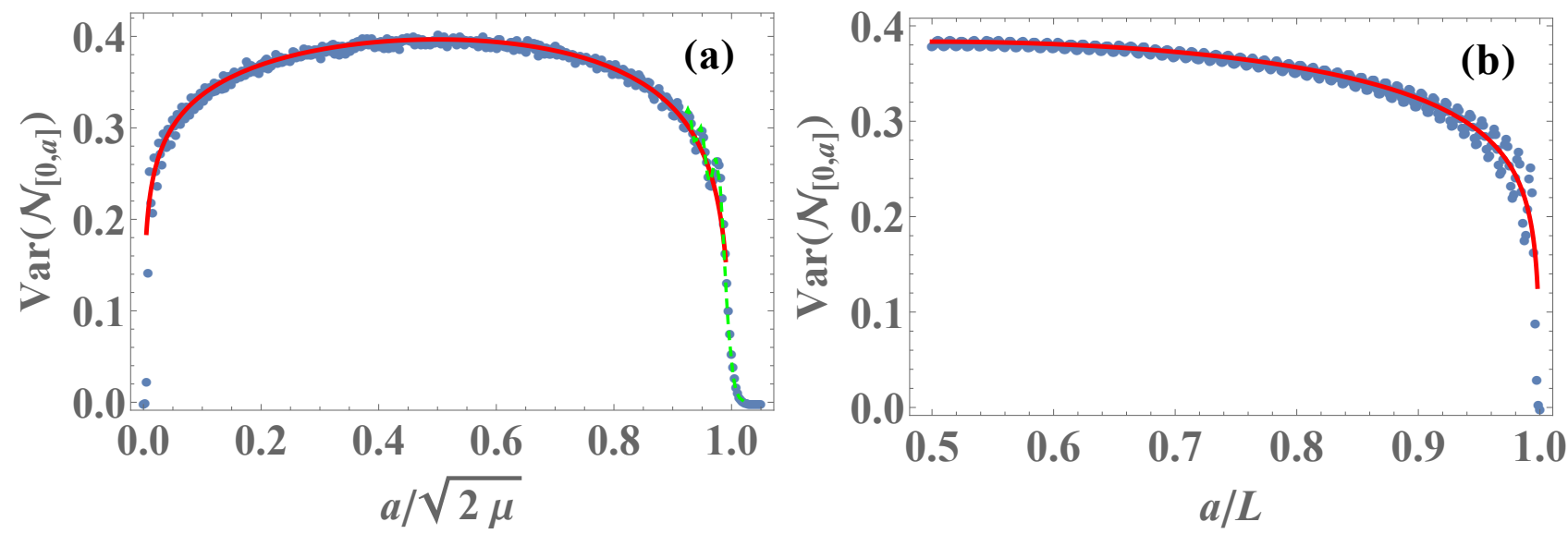

FIG. 5. Variance of $\mathcal{N}_{[0, a]}$ for $N=100$ fermions in (a) the inverse square potential $V(x)=\frac{x^{2}}{2}+\frac{\alpha(\alpha-1)}{2 x^{2}}$ with $\alpha=5 / 2$, and (b) the hard-box potential on the interval $[0, L]$. The symbols, corresponding to numerical simulations in (a) and a numerical evaluations of the integral (S7) in (b), show excellent agreement with our predictions: for (a), with equation (21) in the main text with $\lambda=0$ and $\tilde{a}=a / \sqrt{2 \mu}$ in the bulk, and the scaling form (S66) near the edge, and for (b), with Eq. (S26).

Finally we checked numerically our formula for $K_{\mu}(x, y)^{2}(15)$ in the main text, see discussion in Section IV, for the HO in $d=1$ (in which case $\theta_{x}=\arccos (-x / \sqrt{2 N})$ ). The prediction reads $K_{\mu}(x, y)^{2} \simeq A_{\mu}(x, y)^{2} / 2$, up to rapidly 

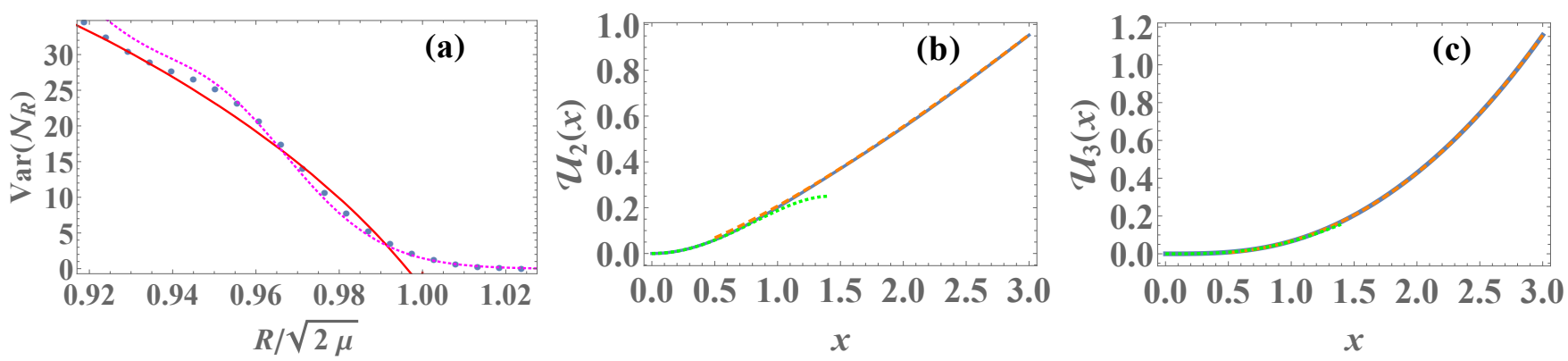

FIG. 6. (a) A zoom in on the edge regime $\tilde{R} \simeq 1$ of Fig. 1 of the main text, showing excellent agreement between the numerical data (symbol) and the theoretical edge prediction, (28) in the text (dotted line). The data was computed over $2 \times 10^{6}$ simulated realizations of the fermions' radial coordinates $r_{1}, \ldots, r_{N}$. All data points were generated over the same set of simulations. (b) and $(c)$ The scaling functions $\mathcal{U}_{2}(x)$ and $\mathcal{U}_{3}(x)$ respectively, that describe the variance of the number of free fermions inside a disc in $d=2$ and a sphere in $d=3$, see Eqs. (S49) and (S51) respectively (blue solid lines respectively). Also plotted are their asymptotic behaviors $\mathcal{U}_{2}(x \ll 1) \simeq x^{2} / 4-x^{4} / 16$ and $\mathcal{U}_{3}(x \ll 1) \simeq 2 x^{3} /(9 \pi)-4 x^{6} /\left(81 \pi^{2}\right)$ (green dotted lines) which describe a Bernoulli distribution of $\mathcal{N}_{\mathcal{D}}$, and $\mathcal{U}_{2}(x \gg 1) \simeq x\left(\ln x+\gamma_{E}-2+5 \ln 2\right) / \pi^{2}$ and $\mathcal{U}_{3}(x \gg 1)$ given in Eq. (S52) (orange dashed lines) which correspond to the macroscopic limit.

oscillating terms which average to zero on scales larger than microscopic, with, for the HO

$$
A_{\mu}(x, y)=\frac{\sqrt{1-\frac{x y}{2 N}}}{\pi(x-y)\left(1-\frac{x^{2}}{2 N}\right)^{1 / 4}\left(1-\frac{y^{2}}{2 N}\right)^{1 / 4}} .
$$

Eq. (S85) has been obtained before in the context of GUE random matrices [100-102]. In Fig. 7 the kernel $K_{\mu}$, rescaled by the amplitude $A_{\mu}$, is plotted as a function of $y$, for $N=200$ and $x=-10$. The result oscillates rapidly as a function of $y$, with an amplitude very close to unity. The quality of this approximation improves as $N$ is increased (not shown). At $|x-y| \ll \sqrt{N}$ the amplitude (S85) matches smoothly with the amplitude $[\pi(x-y)]^{-1}$ of the oscillations predicted by the sine kernel

$$
K_{\mu}(x, y) \simeq \frac{\sin \left(k_{F}(x)|x-y|\right)}{\pi|x-y|}
$$




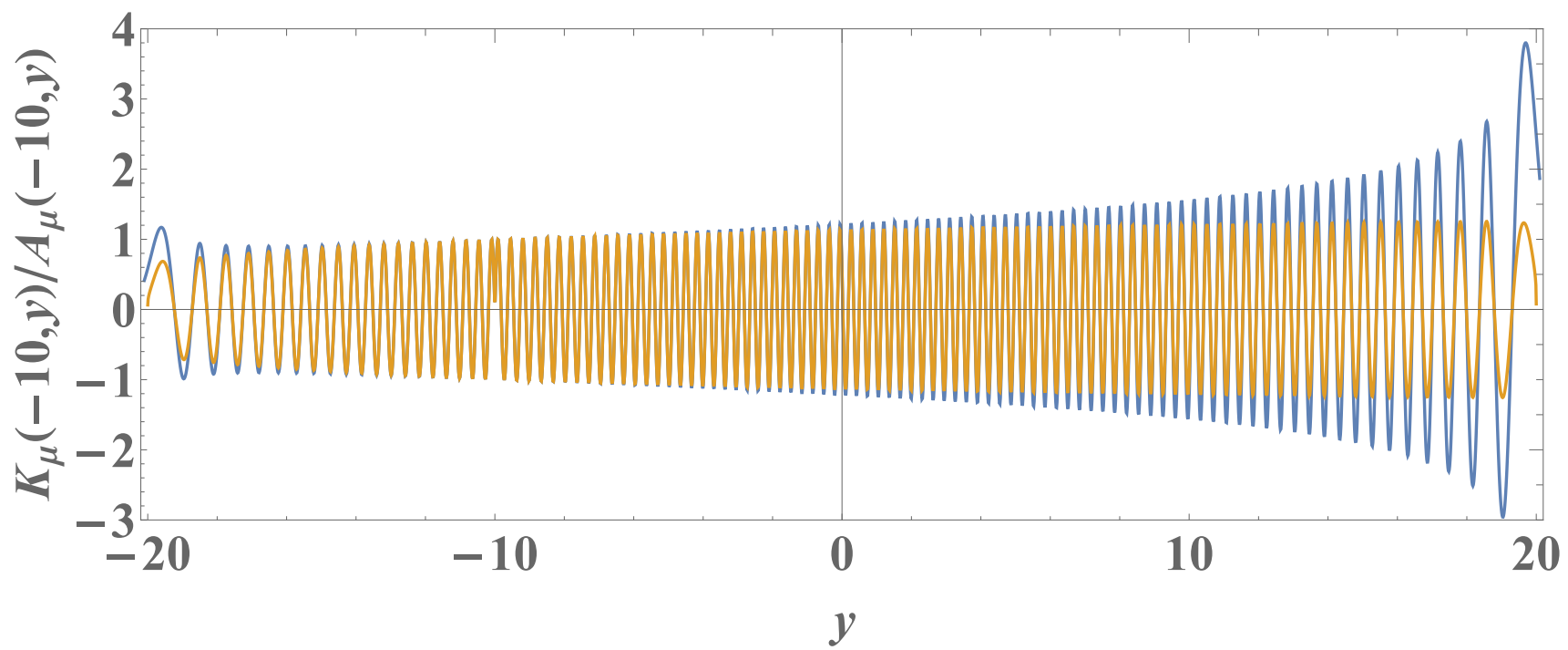

FIG. 7. Orange line: The kernel $K_{\mu}(x, y)$ of the harmonic oscillator in $d=1$, see Eq. (S17), divided by the amplitude in (S85), as a function of $y$, for $N=200$ and $x=-10$. The result oscillates rapidly with an amplitude very close to unity, for all $y$ in the bulk $|y|<\sqrt{2 \mu}$. At $y \simeq x$ it agrees with $K_{\mu}(x, y) / A_{\text {sine }}(x, y)$ where $A_{\text {sine }}(x, y)=[\pi(x-y)]^{-1}$ is the amplitude of the oscillations predicted by the sine kernel, Eq. (S86) (blue line). 